\title{
All-dielectric thermonanophotonics
}

\author{
George P. Zograf, ${ }^{1}$ Mihail I. Petrov, ${ }^{1}$ \\ Sergey V. Makarov, ${ }^{1}$ and Yuri S. Kivshar ${ }^{1,2, *}$
}

April 6, 2021

in

\author{
1 Department of Physics, ITMO University, Saint-Petersburg, 197101, \\ Russia \\ ${ }^{2}$ Nonlinear Physics Centre, Australian National University, Canberra ACT \\ 2601, Australia \\ *yuri.kivshar@anu.edu.au
}

\section{Abstract}

Nanophotonics is an important branch of modern optics dealing with lightmatter interaction at the nanoscale. Nanoparticles can exhibit enhanced light absorption under illumination by light, and they become nanoscale sources of heat that can be precisely controlled and manipulated. For metal nanoparticles, such effects have been studied in the framework of thermoplasmonics which, similar to plasmonics itself, has a number of limitations. Recently emerged all-dielectric resonant nanophotonics is associated with optically-induced electric and magnetic Mie resonances, and this field is developing very rapidly in the last decade. As a result, thermoplasmonics is being replaced by all-dielectric thermonanophotonics with many important applications such as photothermal cancer therapy, drug and gene delivery, nanochemistry, and photothermal imaging. This review paper aims to introduce this new field of non-plasmonic nanophotonics and discuss associated thermally-induced processes at the nanoscale. 


\section{Contents}

1 Introduction 4

2 Fundamentals of optical heating at the nanoscale

2.1 Ultrafast optical heating: Two-temperature regime . . . . . . 7

2.2 Slow optical heating: One-temperature regime . . . . . . . . . 9

3 Optical heating of dielectric nanoparticles 14

3.1 Absorption of light by resonant nanoparticles . . . . . . . . . . 14

3.1.1 A brief summary of the Mie theory . . . . . . . . . . 14

3.1.2 Mechanism of heat generation . . . . . . . . . . 16

3.2 Temperature distribution in nanostructures . . . . . . . . . . 18

3.2.1 Spherical nanoparticles in homogeneous media . . . . . 18

3.2.2 Effect of substrate: non-uniform thermal field distri-

bution ................. . . 19

3.3 Effect of radiative losses on optical heating . . . . . . . . . 20 20

3.4 Effect of nonradiative losses on optical heating . . . . . . . . 24

3.4.1 Losses in natural materials . . . . . . . . . . . . . . . 24

3.4.2 Doping of resonant dielectric nanoparticles . . . . . . 25

3.4.3 Loss control via multiphoton absorption . . . . . . . 29

3.5 Variation of physical properties at elevated temperatures . . . 30

3.6 Nonuniform near-field distribution . . . . . . . . . . . . . . . . 30

4 Thermometry with all-dielectric nanoantennas 33

4.1 Raman scattering . . . . . . . . . . . . . . . 33

4.2 Photoluminescence . . . . . . . . . . . . . 34

4.3 Nonlinear scattering . . . . . . . . . . . . . . . 36 36

4.4 Comparison of different nanothermometers . . . . . . . . 37

5 Applications of all-dielectric thermonanophotonics

5.1 Basic properties of materials employed for thermonanophotonics 38

5.2 Optical cooling . . . . . . . . . . . . . . 40

5.3 Solar energy and heat conversion . . . . . . . . . . . 44

5.4 Biomedical applications . . . . . . . . . . . . . . 45

5.5 Thermorefractive optical nonlinearities . . . . . . . . . . . 47

5.6 Thermally-induced phase transitions . . . . . . . . . . 54

5.7 Optical reshaping . . . . . . . . . . . . . . 57 
6 Conclusion and outlook 


\section{Introduction}

Nanophotonics deals with optically resonant nanostructures, and it provides useful tools to control light at the nanoscale. For many years, nanophotonics was associated with metallic structures and their ability to support surface plasmon polaritons, or plasmons, which are hybrid modes created by coupling of electromagnetic waves to free electrons in metals. Metallic nanostructures supporting plasmons are well-known for their ability to achieve extreme light localization [1], enhance emission [2], manipulate scattering of light [3], demonstrate nonlinear effects [4], and also improve efficiencies of solar cells [5].

Energy of incident light can be localized efficiently in resonant nanostructures, and this results in heating of the nanostructures [6]. A branch of plasmonics that studies thermal effects was shaped as an independent field named thermoplasmonics. It describes many novel effects and has unique applications such as photothermal therapy [7], catalysis [8], reshaping of optical responses [9], and many others.

Conversion of intense energy of light into thermal heat in plasmonic nanostructures is well described for the steady-state regime which represents continuous-wave illumination [10, 11], and also for the case of pulsed laser heating [12, 13]. One of the main approaches for highly efficient optical heating of plasmonic structures is the excitation of surface plasmon resonances manifested by a sufficient increase of optical absorption. In the case of small $(\mathrm{a} \ll \lambda)$ nanoparticles, the surface plasmon resonance can be tuned gradually via prolongation of the nanoparticle (NP) along one axis covering the whole visible and near-IR region. Owing to very small sizes, plasmonic nanoparticles can find a number of applications.

Optical heating of plasmonic nanostructures is widely used in chemistry for photothermal catalysis [14], water heating [15, 16, 17], thermophotovoltaics [18, 19], data recording [20], surface coloring [21], and many other fields [22].

One of the most important applications of thermoplasmonics is photothermal therapy, which uses local overheating for triggering physiological process with proteins starting from stimulation of their diffusion through cell membrane up to their damage and denaturation [23]. Such local overheating of plasmonic nanoparticles was used for cancer antitumor therapy by means of near-infrared light exposure of the desired region with delivered plasmonic NPs [24, 25]. Recent trends lead to combination of thermoplasmonics with 
various approaches in cancer therapy [26]. Finally, plasmonic nanostructures are efficient in photothermal sensing of notorious COVID-19 [27]. However, these are not the only applications of thermoplasmonics in therapy. For instance, local plasmonic overheating is used for bacterial sterilization [28], skincare of acne [29], hair removal [30] or even retinal treatment [31].

On the other hand, biomedical applications require a control of local temperatures in order to avoid unnecessary hyperthermia. In this regard, plasmonic NPs are usually considered as objects with thermally inactive inherent optical response, such as Raman scattering of photoluminescence (PL). Therefore, additional thermally-responsive coatings or substances were employed to estimate the local temperature around various metal nanostructures [32, 33. However, recently a method of thermally-sensitive anti-Stokes PL in gold nanorods which can be used to measure the local temperature was proposed [34]. On this way, further progress on dramatic enhancement of PL efficiency form metal nanoparticle should be done to make it suitable for above mentioned thermoplasmonics applications.

All-dielectric nanophotonics has been rapidly developing for the last several years [35, 36]. Pursuing new high-efficient compact optical systems, the research has been mainly driven by resonant high-refractive index dielectric and semiconductor nanostructures, which are relieved of parasitic Ohmic losses inherent by plasmonics. On this way, outstanding progress has been achieved recently in ultracompact light-emitting systems [37, 38, 39, 40, 41, 42, 43, 44, 45, 46, 47, 48, 49, 50], optical sensing [51, 52, 53, 54, 55, 56, 57, 58, 59, 60, 61, 62, nonlinear nanophotonics [63, 64, 65, 66, 67, 68, 69, 70, 71, 72, , and photovoltaics [73. Despite the fact that losses in all-dielectric materials can be negligible in certain spectral ranges, their spectral dispersion is extremely strong, especially in semiconductor materials in the vicinity of direct (or indirect) transition threshold. Being one of the major problems in solid-state optoelectronics, in nanophotonics the optical losses opened a route for efficient optical heating of subwavelength all-dielectric nanostructures. Till recently, the area of thermal nano-optics was fully associated with nanoplasmonics. Indeed, due to the ability for enormous light localization near the metal surface, plasmonics structures suffered from the parasitic side effect of overheating in applications such as sensing, imaging, spectroscopy and photovoltaics. However, in the late 1990s and early 2000s [74, 75] there have been found promising biomedical applications for precise controllable light-induced protein unfolding and temperature probing and imaging for hyper-thermal therapies. Since then, a huge field of nano-optics studying 


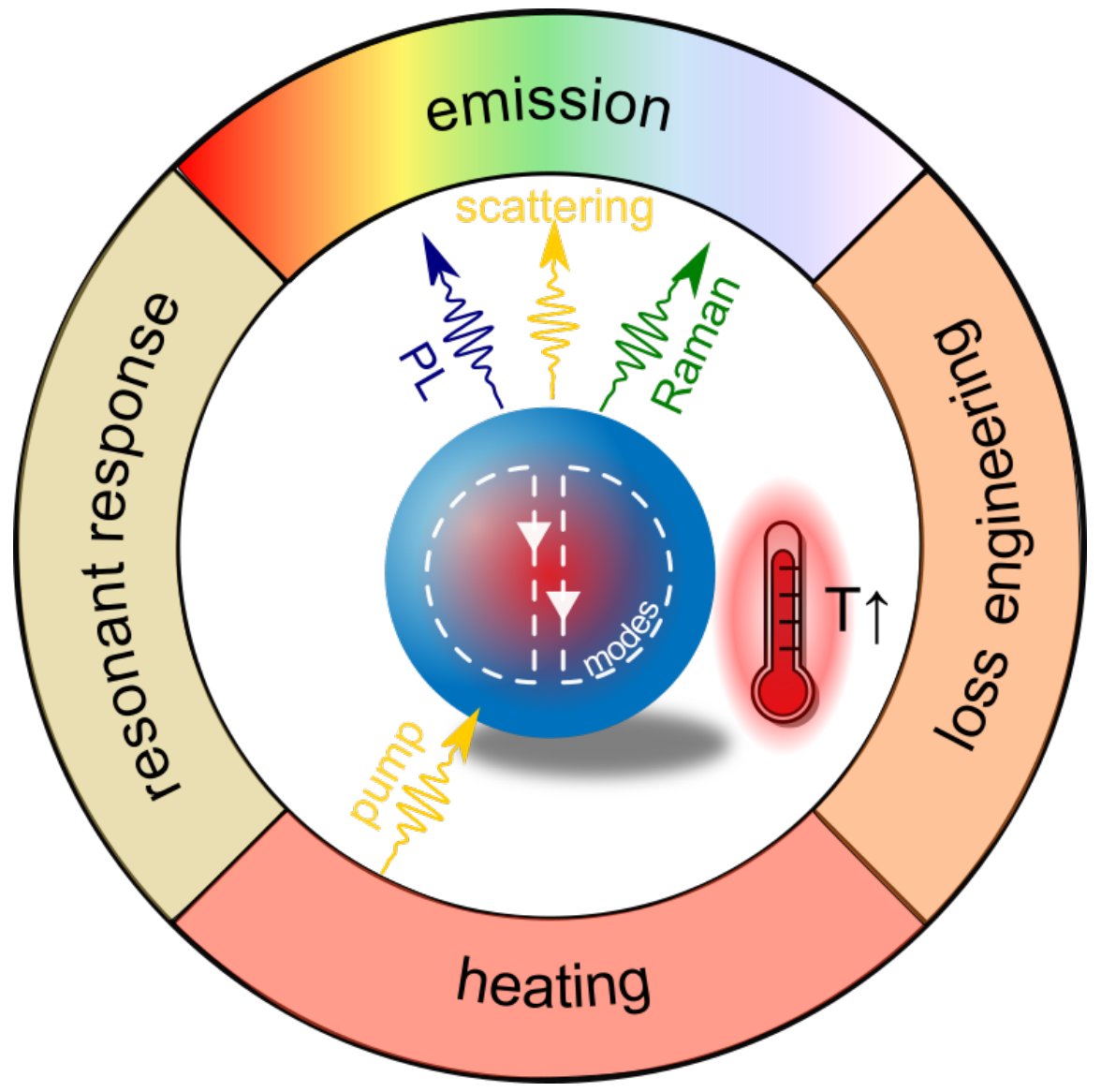

Figure 1: The key concepts underpinning the physics of optical heating of all-dielectric nanoparticles, with the most important effects for optimization and applications. 
nanoscale thermal effects in plasmonics structures has emerged [6, 76].

Some of the applications of thermoplasmonics are already being used in industry, whereas the field of all-dielectric thermonanophotonics based resonant dielectric nanoparticles is new, but it may provide additional degrees of freedom due to a number of features, including

- non-metallic optical materials with a broad range of functionalities;

- low optical losses of dielectric materials precisely tunable from zero to extremely high values;

- strong optical nonlinearity and thermorefractive properties of non-metallic materials.

In this paper, we review the fundamentals of optical heating of subwavelength resonant non-metallic nanostructures and provide a summary of the recent advances in application of dielectric structures for nanoscale heating and thermometry. By replacing partially plasmonic structures and thermoplasmonic effects, all-dielectric thermonanophotonics provides novel degrees of freedom for tuning the optical responses by employing Mie resonances, and it offers in situ temperature detection by means of photoluminescence or Raman scattering. Different strategies to achieve the best performance are provided with the focus on a balance between radiative and non-radiative losses in resonant nanoparticles.

\section{Fundamentals of optical heating at the nanoscale}

Optical heating process represents a cascade of various processes starting from photon absorption and finishing when system relaxes in its initial state after complete cooling.

\subsection{Ultrafast optical heating: Two-temperature regime}

In metals, light is almost exclusively absorbed by free electron transitions within the conduction band. The electron system is thermalized, typically, during 10 fs to 1 ps. Thermalization between the electron subsystem and the lattice is much slower, typically of the order of 1-100 ps, depending on the strength of electron-phonon coupling. Thus, femtosecond laser excitation 
generates a hot electron gas, which than heats the ion (or lattice) sub-system. The coupled nonlinear equations for electrons and lattice can be written, in a more general form, as [77]

$$
\left\{\begin{array}{l}
C_{e} \partial T_{e} / \partial t=\nabla\left(\kappa_{e} \nabla T_{e}\right)-\gamma_{e i}\left(T_{e}-T_{i}\right)+S \\
C_{i} \partial T_{i} / \partial t=\nabla\left(\kappa_{i} \nabla T_{i}\right)+\gamma_{e i}\left(T_{e}-T_{i}\right)
\end{array}\right.
$$

where $T_{e}$ and $T_{i}$ are the electron and lattice temperatures, respectively. $C_{i}$ and $\kappa_{i}$ denote the specific heat capacity and the thermal conductivity of the subsystem $j(j=e, i)$ respectively. The laser pulse is modeled as a source term, $S$, particular for each material. Laser light is absorbed by the electronic subsystem which transfers the absorbed energy to the lattice through electron-phonon collisions represented by the coupling factor $\gamma_{e i}$.

In dielectrics and semiconductors, ultrafast thermal modeling should involve the adequate mechanisms of the photoexcited electron generation and recombination. The electron density in the conduction band $N_{e}$ should be calculated considering absorption term, carrier diffusion, impact ionisation and Auger recombination by solving the following partial differential equation:

$$
\partial N_{e} / \partial t=\nabla\left(k_{B} T_{e} \mu_{e} \nabla N_{e}\right)+G_{e}-R_{e}
$$

the term $G_{e}$ describes the electron generation rate (can be nonlinear), and $R_{e}$ corresponds to relaxation processes such as Shockley-Read-Hall effect, radiative recombination and Auger recombination. The first term on the righthand side of Eq. 2 describes the carrier transport due to diffusion where $k_{B}$ is the Boltzmann constant and $\mu_{e}$ is the electron mobility in the conduction band. Also, the Eq. 2 determines such a basic property as electron specific heat capacity:

$$
C_{e}=\frac{3}{2} k_{B} N_{e}
$$

and electron-phonon coupling factor [78, 79]:

$$
\gamma_{e i}=\frac{C_{e}}{\tau_{\gamma}}=\frac{C_{e}}{\tau_{0}\left[1+\left(N_{e} / N_{t h}\right)^{2}\right]}
$$

where $\tau_{0}$ is the hot carrier relaxation time and $N_{t h}$ is the critical carrier density for screening of electron-phonon interaction. It means that the screening increases total effective time of electron-phonon relaxation in semiconductors $\tau_{\gamma}$. For example, in such semiconductors as $\mathrm{Si}$ and $\mathrm{GaAs}, \tau_{\gamma}$ is typically 
between 0.1 ps and 2 ps depending on photogenerated free electron density [79, 80].

Figure 2 a shows solution of Eqs 14 for Si surface irradiated by an intense fs-laser pulse, where typical scenario of ultrafast electron subsystem heating and subsequent energy transfer to lattice is presented. Remarkably, temperatures of electron and lattice become equal at the picosecond scale. However, further cooling of the heated bulk material is strongly dependent on its thermal conductivity.

Cooling rate $K_{c}$ of semiconductor-based nanoparticles supporting optical resonances is around $0.1-1 \mathrm{~K} / \mathrm{ps}$ [81, 82], being strongly dependent on thermal conductivity of the substrate or hosting medium. Cooling rate of plasmonic nanoparticles under pulsed illumination is discussed in detail elsewhere [76]. As a result, despite the ultrafast character of optical heating upon fs-laser pulses, the effect of temperature localization at nanoscale can be diminished due to too high repetition rate of the coming laser pulses.

\subsection{Slow optical heating: One-temperature regime}

In many cases and practical applications, quasi-CW and CW optical heating are more preferable or realistic regimes of optical heating. In this regard, it is crucial to understand what are the stages in the time-evolution of gradual temperate growth and what are the main limitations in this process.

For solving a problem of optical heating lets start with general equation for the lattice temperature only:

$$
\rho c_{p} \partial T / \partial t=\nabla(\kappa \nabla T)+(\partial Q / \partial t)
$$

where $\rho$ is material density, $c_{p}$ is heat capacity, $\kappa$ is thermal conductivity, $T$ is temperature and $Q$ is the amount of heat received per unit volume of material. Temperature dependence of $\rho, c_{p}, \kappa$, and $(\partial Q / \partial t)$ is negligible in many cases. Thus, Eq.5 can be rewritten to the form:

$$
\partial T / \partial t=\chi \Delta T+\left(\rho c_{p}\right)^{-1}(\partial Q / \partial t)
$$

where $\chi=\kappa / \rho c_{p}$ is thermal diffusivity. Laser beam propagation along the axis $\mathrm{z}$ and fall on xy plane of material create volume heat source:

$$
\partial Q / \partial t=\alpha I(r, t)
$$


(a)
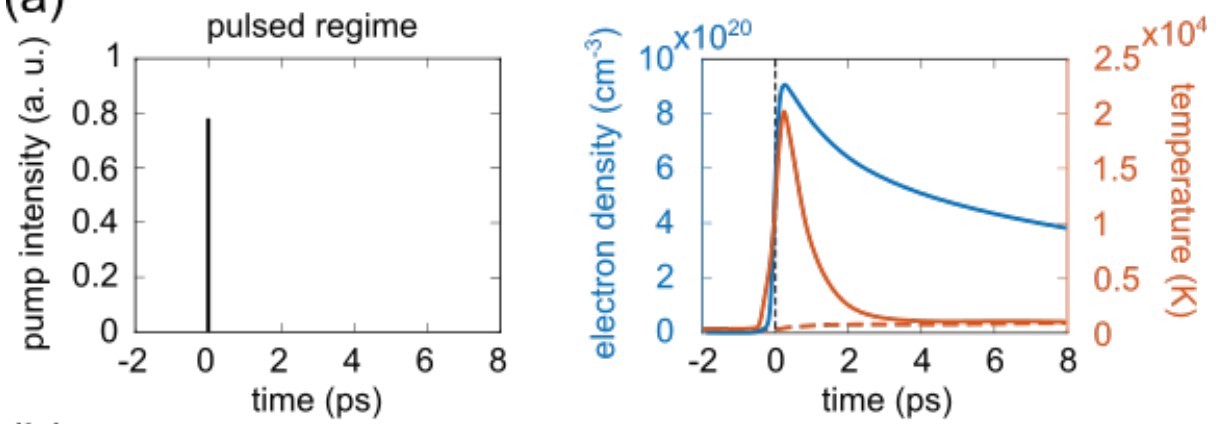

(b)
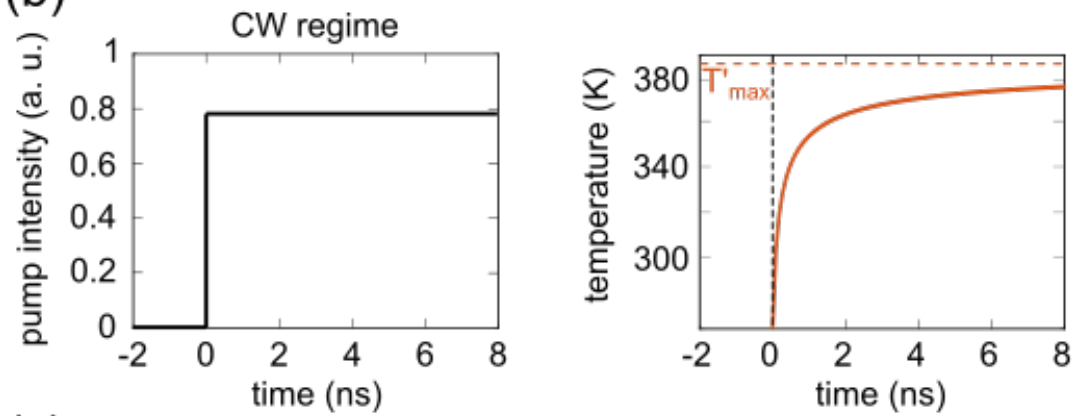

(c)
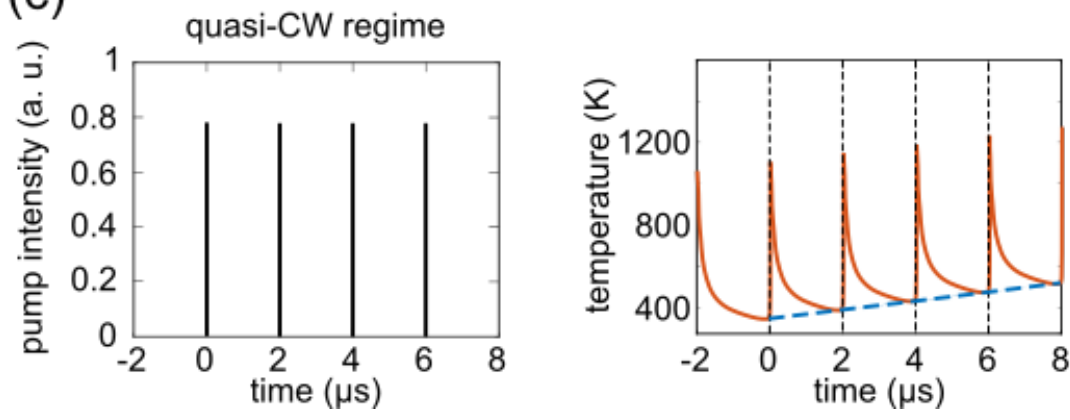

Figure 2: Regimes of optical heating. (a) Calculated carrier number density, carrier temperature, and lattice temperature for bulk silicon surface irradiated by 350 -fs laser pulse with fluence $\sim 0.5 \mathrm{~J} / \mathrm{cm} 2$ schematically shown in upper inset. 83] (b) Calculated solution of Eqs. 16 17 for the case of silicon surface irradiated by $515-\mathrm{nm} \mathrm{CW}$ laser with intensity $50 \mathrm{~mW} / \mu \mathrm{m}^{2}$ and Gaussian beam radius $0.7 \mu \mathrm{m}$. (c) Calculated surface maximum temperature of bulk silicon versus time upon irradiation by a train of 350-fs laser pulses with fluence $\sim 0.5 \mathrm{~J} / \mathrm{cm} 2$ coming with repetition rate $500 \mathrm{kHz}$ schematically shown in upper inset. Red dash line indicates growth of background temperature 83 . 
where $\alpha$ is absorption coefficient and $I(r, t)$ is distribution of light intensity (in region zi0). One of the most widely used case is Gaussian beam, which can be written as:

$$
I(r, t)=(1-R) I_{0} \exp (-\alpha z) \exp \left[-\left(x^{2}+y^{2}\right) / a^{2}\right] f\left(t / \tau_{p}\right),
$$

where $I_{0}$ is the intensity of radiation incident on the absorbing medium from the outside along $z$ direction, $R$ is optical reflection coefficient and $a$ is radius of Gaussian beam. Function $f\left(t / \tau_{p}\right)$ is a general form of temporal shape of laser pulse with duration $\tau_{p}$.

If Eq. 5 is linear, it is also valid for the increase of the sample temperature $T^{\prime}=T-T_{0}$, where $T_{0}$ is the sample temperature before the optical irradiation. Also, in many cases, the process of heating a material can be considered in the absence of heat exchange with the environment using the following boundary condition:

$$
\left.\chi \frac{\partial T^{\prime}}{\partial z}\right|_{z=0}=0
$$

Method of Green function is convenient for solving Eq.5. Indeed, let's assume that the Green function $G\left(r-r^{\prime}, t-t^{\prime}\right)$ is the solution for a heat source perfectly localized in $r=0$ and in the form of instant pulse (i.e. defined by delta-functions $\delta$ ):

$$
\frac{1}{\rho c_{p}}\left(\frac{\partial Q}{\partial t}\right) \rightarrow A \delta\left(\boldsymbol{r}-\boldsymbol{r}^{\prime}\right) \delta\left(t-t^{\prime}\right)
$$

When Eq.6 with boundary conditions Eq.9 is linear, the solution of the heat problem with arbitrary source has the form:

$$
T^{\prime}(r, t)=\frac{1}{\rho c_{p}} \int_{-\infty}^{t} d t^{\prime} f\left[\frac{\partial Q}{\partial t}\left(r^{\prime}, t^{\prime}\right)\right] G(r-r, t-t) d^{3} r^{\prime}
$$

For a point source, Eq.6 can be rewritten as:

$$
\partial T^{\prime} / \partial t=\chi \Delta T^{\prime}+A \delta(r) \delta(t)
$$

Solving this equation with taking into account the boundary conditions gives the following expression:

$$
T^{\prime}(r, t)=\frac{A}{(4 \pi \chi t)^{3 / 2}} \exp \left(-\frac{r^{2}}{4 \chi t}\right)
$$


According to this expression, after instant point-like heat source switching on, the temperature rise in the heated area has temporal dependence like $T^{\prime} \sim t^{-3 / 2}$, while characteristic size of the heated zone grow with time as $\sim(\chi t)^{1 / 2}$.

General solution of Eq 6 for the continuous wave laser excitation with Gaussian intensity profile (see Eq.8) can be written as:

$$
\begin{aligned}
& \frac{\partial T^{\prime}}{\partial t}=\frac{\alpha(1-R) I_{0}}{2 \rho_{0} c_{p}} \frac{1}{1+4 \chi t / a^{2}} \exp \left(-\frac{x^{2}+y^{2}}{a^{2}+4 \chi t}\right) \exp \left(\alpha^{2} \chi t\right)\left\{\exp (\alpha z) \operatorname{erfc}\left[\alpha(\chi t)^{1 / 2}+\frac{z}{(4 \chi t)^{1 / 2}}\right]+\right. \\
& \left.+\exp (-\alpha z) \operatorname{erfc}\left[\alpha(\chi t)^{1 / 2}-\frac{z}{(4 \chi)^{1 / 2}}\right]\right\} \theta(t)
\end{aligned}
$$

where $\theta(t)$ is Heaviside function and erfc $x=\frac{2}{\sqrt{\pi}} \int_{x}^{\infty} e^{-t^{2}} \mathrm{~d} t$ is complementary error function.

The maximum rate of temperature increase is on the surface of the irradiated substrate $(z=0)$ and on the axis of the laser beam incidence $(x=y=0)$ :

$$
\frac{\partial T^{\prime}}{\partial t}(r=0, t)=\frac{\alpha(1-R) I_{0}}{\rho_{0} c_{p}} \frac{\exp \left(\alpha^{2} \chi t\right) \operatorname{erfc}\left[\alpha(\chi t)^{1 / 2}\right]}{1+4 \chi t / a^{2}}
$$

At the initial stage $\left(t \leqslant \min \left\{a^{2} / \chi, 1 / \alpha^{2} \chi\right\}\right)$ thermal conductivity does not affect the heating rate, which is almost constant $\left(\partial T^{\prime} / \partial t=\alpha(1-R) I_{0} / \rho_{0} c_{p}\right.$ from Eq. 15 at $t=0)$. The physical meaning of the quantity $a^{2} / \chi$ is the characteristic time during which the temperature transfer occurs up to the distance corresponding to lateral size of the laser beam $a$.

For the case of highly absorbing materials like metals or semiconductors at wavelengths in the interband absorption range, penetration depth of light in the material $\left(d=\alpha^{-1}\right.$ absorption length) manifests itself in the sample heating after some characteristic time $d^{2} / \chi=1 / \alpha^{2} \chi$. Thus, at the times $t \leqslant \min \left\{a^{2} / \chi, 1 / \alpha^{2} \chi\right\}$ the maximum temperature of the substrate increases over time according to the linear law: $T^{\prime} \sim t$ as follows from Eq.14.

Let us assume that the transverse size of the laser beam is significantly greater than the absorption length $(a \gg d)$. Then, with time increasing the heat transfer into the medium "switch on" of the first time is included which reduces the rate of heating. For the condition $1 / \alpha^{2} \chi \leqslant t \leqslant a^{2} / \chi$ Eq. 15 takes the form, where the temperature increment grows sub-linearly $\left(T^{\prime} \sim t^{1 / 2}\right)$. During the time $t \geq a^{2} / \chi$ after the laser exposure, thermal conductivity in the direction along the surface begins to influence the temperature increase. Heating rate decreases rapidly. At $t \geq a^{2} / \chi$ the solution for temperature 
$t \gg a^{2} / \chi$ is established by equation:

$$
T^{\prime} \approx T_{\max }^{\prime}-\frac{(1-R) I_{0} \pi a^{2}}{2 \rho_{0} c_{p} \pi \chi(4 \pi \chi t)^{1 / 2}}
$$

The maximum temperature $T_{\max }^{\prime}$ are defined as integral Eq.15. $T_{\max }^{\prime}=$ $T^{\prime}(r=0, t=\infty)$. In case $\alpha a \gg 1$, it can be estimated as:

$$
T_{\max }^{\prime} \sim \frac{(1-R) I_{0} a}{\rho_{0} c_{p} \chi}=\frac{(1-R) \mathfrak{P}}{\kappa a},
$$

where $\mathfrak{P}=a^{2} I_{0}$ is total power of Gaussian beam with $a$ radius and $I_{0}$ intensity.

For the opposite case, when $(d \gg a)$ slowing of the heating rate starts at $t \geq a^{2} / \chi$ and yielding the logarithmic trend $T^{\prime} \sim \ln (t)$, when the relatively slow temperature growth caused by more efficient lateral heat flow to the bulk. Further heating in the regime of $t \gg 1 / \alpha^{2} \chi$ leads to saturation of temperature following the law Eq 16 at $\ln (1 / \alpha a) \gg 1$ with maximum temperature:

$$
T_{\max }^{\prime} \sim \frac{(1-R) \mathfrak{P}}{\kappa} \alpha \ln \frac{1}{\alpha \mathrm{a}},
$$

Solution of Eq. 16 for Si substrate and 515-nm CW laser with intensity $50 \mathrm{~mW} / \mu \mathrm{m}^{2}$ and Gaussian beam radius $0.7 \mu \mathrm{m}$ is given in Fig. 2b. The maximum temperature increase from the $273 \mathrm{~K}$ in this case is around $120 \mathrm{~K}$, which is at least one order of magnitude less than that for the pulsed regime (see Fig. 2a).

In many cases, laser irradiation of targets is carried out by train of short pulses. This regime is a mixture of pulsed optical heating and slow continuous growth of temperate, which is usually called as quasi continuous wave (quasi$\mathrm{CW}$ ) regime. In Fig. 2p, the calculated optical heating of Si substrate is shown for the case of fs-pulse train with inter-pulse distance $2 \mu \mathrm{s}$, revealing considerable and relatively slow heat accumulation after each pulse. Such an increase of background temperature is governed mainly by average intensity of incident light both inter-pulse duration and

Also, it is important to mention that strong thermal localization in zdirection (i.e. in the case of $a \gg d$ ) yields much more efficient optical heating. Further optimization can be done with light source localization in lateral direction by reducing $a$. However, the diffraction limit does not allow for strong and local optical heating, making it crucial to employ nanoparticles. 
On this way, one of the most crucial differences between the optical heating of plain surface and nanoparticles is in importance of the optical losses. Indeed, on one hand, for the case of surface, higher absorption is almost always leads to stronger heating, whereas in resonant nanoparticles. In opposite, the most optimal cases for optical heating of the resonant particles can be achieved with low-loss materials, which we discuss in the next Sections.

\section{Optical heating of dielectric nanoparticles}

In order to understand the mechanism of resonant optical heating of optically resonant nanoparticle, one should study the light absorption cross-section of the nanoscale object. For this purpose, we consider light scattering by a spherical nanoparticle in homogeneous loss-free environment as basic primer widely utilized in nanophotonics. The exact solution of this electromagnetic problem was described by Gustav Mie back in the early XX ${ }^{\text {th }}$ century, when he solved the problem on the plane wave scattering by a subwavelength sphere or infinite cylinder [84]. The detailed solution of the Mie problem is provided elsewhere [85].

\subsection{Absorption of light by resonant nanoparticles}

\subsubsection{A brief summary of the Mie theory}

In general case, a dielectric spherical resonator supports, so-called, Miemodes of both electric and magnetic nature. The total scattering $C_{s c a}$, extinction $C_{\text {ext }}$ and absorption $C_{a b s}$ cross-section with predefined incident intensity $I_{i}$, wave vector $k$ are defined as follows

$$
\begin{array}{r}
C_{s c a}=\frac{2 \pi}{k^{2}} \sum_{l=1}^{\infty}(2 l+1)\left(\left|a_{l}\right|^{2}+\left|b_{l}\right|^{2}\right) \\
C_{\text {ext }}=\frac{2 \pi}{k^{2}} \sum_{l=1}^{\infty}(2 l+1) \operatorname{Re}\left(a_{l}+b_{l}\right) \\
C_{a b s}=C_{e x t}-C_{s c a},
\end{array}
$$

where $a_{l}$ are the electric modes and $b_{l}$ are the magnetic ones, that are defined as: 


$$
\begin{aligned}
a_{l} & =\frac{m \psi_{l}(m x) \psi_{l}^{\prime}(x)-\psi_{l}(x) \psi_{l}^{\prime}(m x)}{m \psi_{l}(m x) \xi_{l}^{\prime}(x)-\xi_{l}(x) \psi_{l}^{\prime}(m x)} \\
b_{l} & =\frac{\psi_{l}(m x) \psi_{l}^{\prime}(x)-m \psi_{l}(x) \psi_{l}^{\prime}(m x)}{\psi_{l}(m x) \xi_{l}^{\prime}(x)-m \xi_{l}(x) \psi_{l}^{\prime}(m x)},
\end{aligned}
$$

and Riccati-Bessel functions are:

$$
\psi_{l}(\rho)=\rho j_{l}(\rho) \quad \xi_{l}(\rho)=\rho h_{l}^{(1)}(\rho) .
$$

For the following coefficients $x$ is the diffraction parameter, $\rho=k r$, and $m$ is the relative refractive index.

$$
\begin{aligned}
x=k a=\frac{2 \pi \mathrm{n} a}{\lambda} & m=\frac{k_{1}}{k}=\frac{\mathrm{n}_{1}}{\mathrm{n}} \\
j_{l}(\rho)=\sqrt{\frac{\pi}{2 \rho}} J_{l+1 / 2}(\rho) & y_{l}(\rho)=\sqrt{\frac{\pi}{2 \rho}} Y_{l+1 / 2}(\rho)
\end{aligned}
$$

$J_{n}(\rho)$ and $Y_{n}(\rho)$ are Bessel functions of first and second order.

The typical scattering and absorption cross-section spectra obtained with help of Eq. 19 are shown in Fig. 3. The obtained spectra take into account only a single magnetic dipole (MD) eigenmode of a spherical nanoparticle of $215 \mathrm{~nm}$ diameter corresponding to the coefficient $b_{1}$ only in Eq. 20. One can see that the spectra have a resonance at a wavelength $\lambda_{0}=2 \pi c / \omega_{0}$ corresponding to the fundamental MD Mie mode. The position of the resonance can be roughly estimated from the condition $2 \pi R / \lambda_{0} \approx 1$. While the real part of the permittivity $\varepsilon^{\prime}$ is fixed at 15.3 which roughly corresponds to parameters of crystalline silicon in the visible range, the imaginary part is varied: $\varepsilon^{\prime \prime}=0$ (Fig. 3a), $\varepsilon^{\prime \prime}=0.28$ (Fig. 3b), $\varepsilon^{\prime \prime}=1$ (Fig. 3c), and $\varepsilon^{\prime \prime}=4$ (Fig. $\left.3 \mathrm{~d}\right)$. The increase of imaginary part $\varepsilon^{\prime \prime}$ provides the increase of non-radiative losses of the MD mode characterized by the rate $\gamma_{\text {nrad }}$, while the radiative losses channel is always present and its rate $\gamma_{\text {rad }}$ scales with the size $\gamma_{\mathrm{rad}} / \omega_{0} \sim n^{3}$, where $n$ is the refractive index of the material.

The gradual increase of Ohmic losses $\gamma_{\text {nrad }}$ results in broadening of the scattering spectra which is schematically shown in the upper row of Fig. 3 with red (radiative loss channel) and green (Ohmic losses channel) arrows. However, one can notice that the absorption spectra, which has the key importance form optothermal effects in nanostructures, has a non-monotonous 

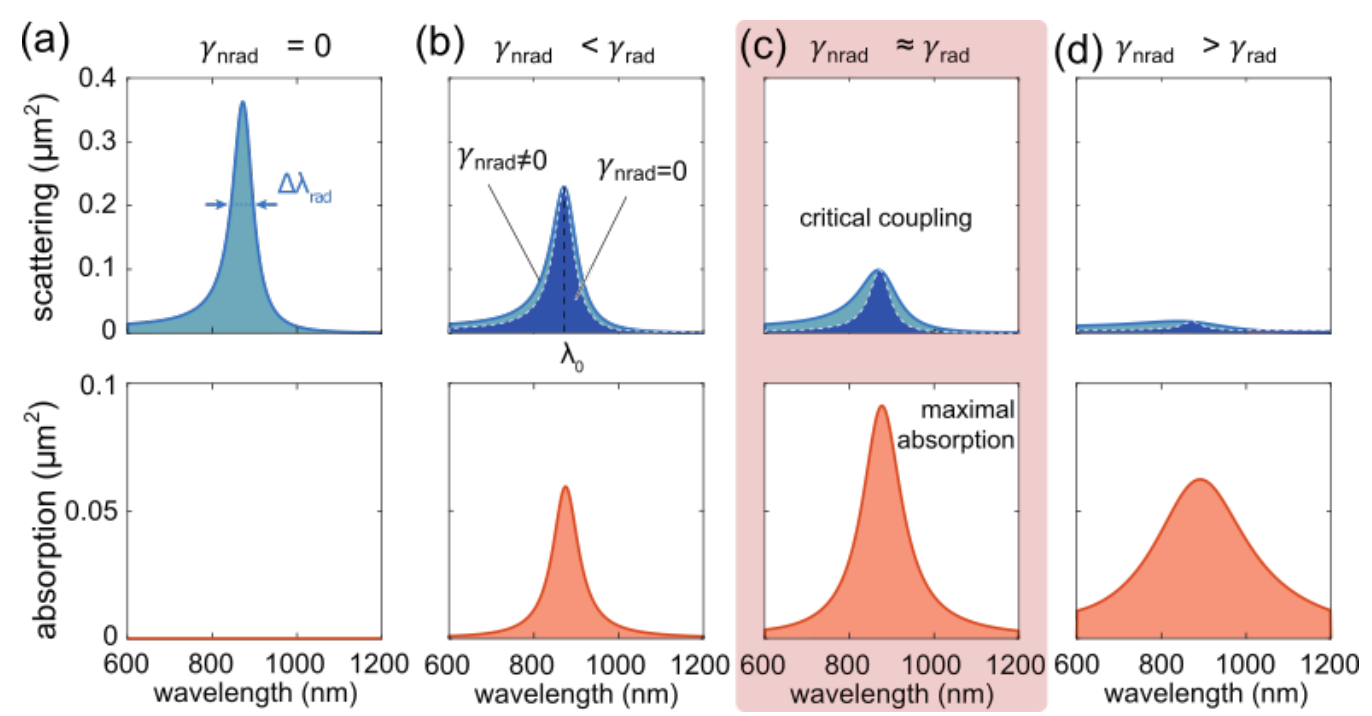

Figure 3: Radiative and Ohmic losses in dielectric Mie resonators. Analytically calculated scattering (upper row) and absorption (lower row) cross-section through Mie theory for spherical nanoparticle of $215 \mathrm{~nm}$ diameter with fixed real part of the permittivity $\varepsilon^{\prime}=15.3$, which correspond to crystalline silicon in the visible range and imaginary part of the permittivity: (a) $\varepsilon^{\prime \prime}=0$, (b) $\varepsilon^{\prime \prime}=0.28$, (c) $\varepsilon^{\prime \prime}=1$, and (d) $\varepsilon^{\prime \prime}=4$.

dependence on the losses, while the scattering intensity gradually decreases. At certain value of $\varepsilon^{\prime \prime}$, further increase of the nonradiative losses results in even decrease of the absorption cross-section. Therefore, the optimized regime is realized in Fig. 3(c), which corresponds to maximal absorption cross section and so-called 'critical-coupling' regime provided by $\gamma_{\mathrm{rad}}=\gamma_{\mathrm{rad}}$ and can condition $C_{s c a}=C_{a b s}$ at the resonance as shown in Fig. 3r. The conclusions in this sections correlated with those described in frameworks of other approaches [86, 87]. Despite that we have considered an example of a spherical particle, the coupling condition can be applied to any resonant nanostructure and its generalized form will be considered below in Section 3.3 .

\subsubsection{Mechanism of heat generation}

The energy absorbed by the nanostructures goes for increasing its the temperature. The density of the heat power $q(\mathbf{r})$ inside the NP can be derived 
basing on the Ohmic absorption light power:

$$
q(\mathbf{r})=\frac{1}{2} \operatorname{Re}\left[\mathbf{J}^{\star}(\mathbf{r}) \cdot \mathbf{E}(\mathbf{r})\right]
$$

where $\mathbf{J}(\mathbf{r})$ is the complex amplitude of the electronic current density inside the NP. As $\mathbf{J}(\mathbf{r})=i \omega \mathbf{P}$ and $\mathbf{P}=\varepsilon_{0}(\varepsilon(\omega)-1) \mathbf{E}$ one ends up with

$$
q(\mathbf{r})=\frac{\omega}{2} \operatorname{Im}(\varepsilon(\omega)) \varepsilon_{0}|\mathbf{E}(\mathbf{r})|^{2}=\frac{1}{2} \sigma|\mathbf{E}(\mathbf{r})|^{2},
$$

where the latter expression is obtained in terms of the electric conductivity $\sigma$ owing to the relation between imaginary part of the permittivity determines the conductivity as $\sigma=\varepsilon_{0} \omega \operatorname{Im}(\varepsilon)$, where $\varepsilon_{0}$ is the dielectric permittivity of vacuum. The total dissipated power $Q$ can then be expressed:

$$
Q=\int_{V} q(\mathbf{r}) \mathrm{d}^{3} r
$$

where the integral runs over the NP volume $V$.

Generally, the larger the volume of an arbitrary nanoparticle, the stronger the heating. In general case, integration in Eq.26 over an arbitrary nanoparticle volume supporting eigenmodes allows to rewrite it in terms of an effective mode volume $V_{\text {eff }}$ and spatially averaged field enhancement factor $<|E|>$, which determines how much energy can be accumulated inside the nanoparticle. Thus, we can rewrite Eq 26 for the absorbed power as [88]:

$$
Q \sim \sigma<|E|>^{2} V_{\text {eff }}
$$

From this expression, on can see that the effective mode volume inside the nanoparticle $V_{\text {eff }}$ is very important for the optical heating of NP. Also, the effective mode volume can be very different from the physical volume of NP. For plasmonic nanoparticles $(\operatorname{Re}(\epsilon)<0)$, the effective volume is defined by a skin depth, which is less than $\delta \approx 20 \mathrm{~nm}$ for most of metals in the visible range [89]. Thus, the effective mode volume of plasmonic nanoparticles is $V_{\text {eff }} \approx \pi D^{2} \delta$. In opposite to metals, dielectrics support optical penetration depth much larger than the diameter of the nanoparticle in the visible range. The effective volumes of Mie-type modes are typically of the order of nanoparticle volume $V_{\text {eff }} \approx \pi D^{3} / 6$. It means that the increase of the nanoparticle size is effective for the temperature increase in the case of dielectrics and less effective for the plasmonic nanoparticles. 
On the other hand, in the case of a spherical geometry in homogeneous media, the absorbed power $Q$ can be calculated basing on the absorption cross-section (see Eq,19) from the Mie theory:

$$
Q=C_{\mathrm{abs}} I_{0}
$$

This expression is quite useful for simple estimation of NP heating basing on the results of Fig, 3, as well as for temperature distribution analysis which we discuss below.

\subsection{Temperature distribution in nanostructures}

\subsubsection{Spherical nanoparticles in homogeneous media}

We now focus on the temperature distribution inside a nanostructure in the steady state regime under CW laser illumination. Assuming that $R$ is the typical size of the nanostructure (sphere as a primer) and thermal conductivity $\kappa$, we consider that is embedded in homogeneous media or placed on substrate with thermal conductivity $\kappa_{s}$. According to Eq.5 the time required to reach the stationary regime in a nanostructure is of the order of $\sim c_{p} \rho R^{2} / \kappa$ which is order of $1 \mu \mathrm{s}$ for $200 \mathrm{~nm}$ particle.

The temperature distribution can be obtained by solving Eq.5 in the whole region of interest, which is valid as soon as diffusion mechanism of heat transport is applicable. Its violation occurs at the nanoscale when the mean free path of phonons $l$ becomes larger than the characteristic nanostructure size $l \gg R$. For the most materials of dielectric nanophotonics the phonon mean free path varies in the range 10-200 $\mathrm{nm}$ depending on the particular phonon models [90], which is comparable with the typical size of nanoparticles. Beyond the heat diffusion regime, one should consider Boltzman transport equation and possible ballistic phonon propagation, which is well summarized in the recent paper [91. The steady-state temperature distribution $T(\mathbf{r})$ inside and outside the NP can be, thus, obtained as a solution of the heat diffusion equation:

$$
\begin{array}{ll}
\nabla \cdot[\kappa \nabla T(\mathbf{r})]=-q(\mathbf{r}) & \text { inside the NP } \\
\nabla \cdot\left[\kappa_{S} \nabla T(\mathbf{r})\right]=0 & \text { outside the NP }
\end{array}
$$

The power density distribution $q(\mathbf{r})$ can be strongly inhomogeneous as the field distribution is defined by the particular structure of the resonant mode. At the same time, the temperature appears to be almost constant 
across the nanoparticle volume [92] in case when the thermal conductivity of the nanoparticle material is much larger than the thermal conductivity of the environment $\kappa \gg \kappa_{s}$ (air, glass, liquid, etc.). Indeed, for a spherical NP of radius $R$, the calculations for homogeneous power density $q(\mathbf{r})=Q / V$ leads to an analytical temperature dependence:

$$
\begin{array}{ll}
T_{\text {out }}=\frac{Q}{4 \pi \kappa_{2} r}+T_{0}, & r>R \\
T_{N P}=\frac{Q}{8 \pi \kappa R}\left(1-\frac{r^{2}}{R^{2}}\right)+\frac{Q}{4 \pi \kappa_{s} R}+T_{0}, & r<R
\end{array}
$$

As one can see, the first term corresponding to temperature non-homogeneity vanishes as soon as $\kappa_{s} \ll \kappa$. Finally, the nanoparticle temperature increase $\Delta T_{\mathrm{NP}}$ can be simplified to the following expression:

$$
\Delta T_{N P}=T_{N P}-T_{0} \approx \frac{Q}{4 \pi \kappa_{s} R}=\frac{C_{\mathrm{abs}} I_{0}}{4 \pi \kappa_{s} R} .
$$

As one can see from Eq. 31, that the temperature elevation is directly defined by the absorption cross section $C_{\text {abs }}$ reaching its maximum in critical coupling regime as shown in Fig. 6 .

Also, one can notice that Eq. 31 resembles the expression for maximum temperature of surface heating Eq. 17, where nanoparticle's radius $R$ can be much smaller than the radius of the diffraction-limited focused Gaussian beam $a$. Moreover, the analytical consideration with Eq 30 was done for the case of a spherical particle in homogeneous surrounded by a uniform media. Nevertheless, it can be easily extended to other geometries by introducing an effective conductivity parameter $\kappa_{s}$ in Eq. 31, which depends on particular geometry of the structure. Baffou et al. have numerically extracted this effective parameter for a number of simple geometries of nanostructures geometries [10].

\subsubsection{Effect of substrate: non-uniform thermal field distribution}

The thermal conductivity of solid materials utilized in nanoplasmonics is normally much higher than of the environment one for aqueous or gas surrounding. In this regard, homogeneous thermal distribution within NPs becomes a good approximation in the most cases. However, this approximation for all-dielectric nanostructures is more critical and it may fail for many particular geometries. For example, if one considers crystalline silicon nanosphere optical heating in homogeneous aqueous media, under the assumptions that

the thermal conductivity of silicon $(156 \mathrm{~W} /(\mathrm{m} \cdot \mathrm{K}))$ is much greater than the 
water's one (order of $\sim 1 \mathrm{~W} /(\mathrm{m} \cdot \mathrm{K})$ ), allows one to use the Eq. 31. However, thermal conductivity of amorphous silicon is two orders of magnitude lower than of crystalline silicon $1.8 \mathrm{~W} /(\mathrm{m} \cdot \mathrm{K})$ and is comparable to glass $(\sim 0.8$ $\mathrm{W} /(\mathrm{m} \cdot \mathrm{K})$ ) often used as a substrate materials in nanofabrication process, while sapphire has even much higher thermal conductivity of $34 \mathrm{~W} /(\mathrm{m} \cdot \mathrm{K})$. Thus, the problem of thermal isolation between nanostructure and the substrate becomes important for effective heating of all-dielectric nanostructures.

Figure 4 shows schematically the temperature distribution inside nanoparticles placed over dielectric substrates. As one can see, the substrate material and the shape of the nanoparticle plays a significant role for thermal transfer under CW laser heating, as was already studied elsewhere [93, 94]. For two cases when nanoparticle made of crystalline silicon of spherical and cylindrical shapes (Fig. 4a,b) the area of thermal contact does not affect the homogeneity of temperature distribution inside the nanoparticle for glass substrate with low thermal conductivity. The opposite case occurs when the substrate is made of sapphire with a significantly larger thermal conductivity. One can see in Fig. 4c, that the upper and bottom parts of the nanocylinder have distinguishable homogeneity in temperature values. For this particular reason, the color-bar is in logarithmic scale to demonstrate more clearly the presence of non-uniform temperature distribution pattern in such case.

\subsection{Effect of radiative losses on optical heating}

Here, we provide the basics of nanoresonators heating. We start with a primer example of a resonators with a single resonant mode in the spectral region of interest. The mode of the resonator can be described within the coupled mode theory. The field inside the resonator $\mathbf{E}(\mathbf{r}, t)=a(t) \mathbf{u}(\mathbf{r}) / \sqrt{\varepsilon_{0}}$, where $a(t)$ is the mode amplitude and $u(\mathbf{r})$ is the mode spatial distribution.

$$
\frac{d a(t)}{d t}=\left(-i \omega_{0}-\gamma\right) a(t)+i \sqrt{\Gamma_{r m}} f(t)
$$

Here, $\omega_{0}$ is the resonant frequency of the mode, $\gamma=\gamma_{r}+\gamma_{n r}$ is the total losses of the mode consisting of radiative $\left(\gamma_{r}\right)$ and nonradiative $\left(\gamma_{n r}\right)$ losses. The system is driven by the external field with mode amplitude $f(t)$ and $\Gamma_{r m}$ is the radiative losses of the resonator into this mode. Assuming a continuous wave excitation, we can consider the spectral representation of the problem 


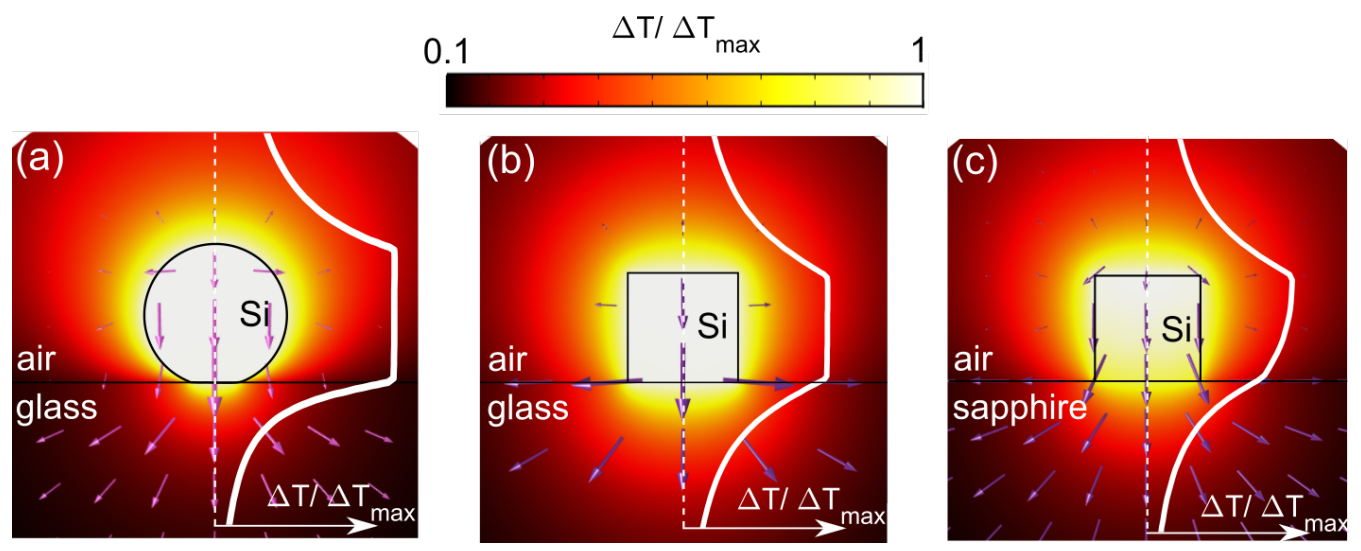

Figure 4: Effect of substrate on temperature distribution in nanoresonators. Numerical simulation of the temperature distribution under CW laser heating of a single silicon (a) nanosphere on a glass substrate, (b) nanocylinder on a glass substrate and (c) nanocylinder on a sapphire substrate. The color-bar bar represents the scale with temperature increase normalized over maximum value of temperature distribution along the structure. White line corresponds to temperature profile along the linear section through the z-axis. Purple arrows demonstrate the heat flux. The arrow size is proportional to the flux magnitude. 


$$
\begin{gathered}
f(t)=\hat{f}(\omega) e^{-i \omega t} \text { and } a(t)=\hat{a}(\omega) e^{-i \omega t}: \\
-i \omega \hat{a}(\omega)=\left(-i \omega_{0}-\gamma\right) \hat{a}(\omega)+i \sqrt{\Gamma_{r m}} \hat{f}(\omega) \Rightarrow \\
\hat{a}(\omega)=\frac{i \sqrt{\Gamma_{r m}} \hat{f}(\omega)}{i\left(\omega_{0}-\omega\right)+\gamma}
\end{gathered}
$$

The optical heating of the nanostructure is provided by the ohmic losses of material. Indeed, the energy dissipated due to Joule heating in the nanostructure can be estimated as follows:

$$
Q=\int_{V} \sigma|E(r)|^{2} d V=|a(t)|^{2} \int_{V} \sigma|u(r)|^{2} d V,
$$

where $\sigma$ is the conductivity of material. The total energy losses can be derived from (32) relying on $Q_{t o t}=d|a|^{2} / d t=-\left(\gamma_{r}+\gamma_{n r}\right)|a|^{2}$, thus $Q=\gamma_{n r}|a|^{2}$, which immediately provides us with the expression for nonradiative losses $\gamma_{n r}=\frac{1}{\varepsilon_{0}} \int_{V} \sigma|u(r)|^{2} d V$.

The temperature, which acquires the resonator in the stationary regime is homogeneous across its volume and has a simple expression:

$$
\Delta T=\frac{Q}{4 \pi \kappa_{s} R}=\frac{1}{4 \pi \kappa_{s} R} \frac{\gamma_{n r} \Gamma_{r m}|\hat{f}(\omega)|^{2}}{\left(\omega_{0}-\omega\right)^{2}+\left(\gamma_{r}+\gamma_{n r}\right)^{2}},
$$

where $\kappa_{s}$ is the thermal conductivity of the surrounding media, and $R$ is the nanoparticle radius. The maximal temperature can be achieved at the resonance:

$$
\Delta T_{\max }=\frac{1}{4 \pi \kappa_{s} R} \frac{\gamma_{n r} \Gamma_{r m}|\hat{f}(\omega)|^{2}}{\left(\gamma_{r}+\gamma_{n r}\right)^{2}}
$$

For a case of a plane wave the amplitude $\hat{f}$ is related to the field intensity as $\hat{f}=\sqrt{c_{0} \varepsilon_{0} \omega R^{2}} E_{0}$.

Critical coupling. One can see that the maximal temperature is limited by the losses factor but also defined by the ratio between radiative and nonradiative losses, which is often referred to as a critical coupling regime: the maximal heating of the structure will be observed one radiative and nonradiative losses will be balanced $\gamma_{r}=\gamma_{n r}=\gamma_{o p t}$. In the critical coupling regime, the maximal temperature will inverse proportional to the overall losses $\Delta T_{\text {max }} \sim 1 / \gamma_{\text {opt }}$, clearly showing that with the decrease of the total losses the heating efficiency will be monotonically increased. 


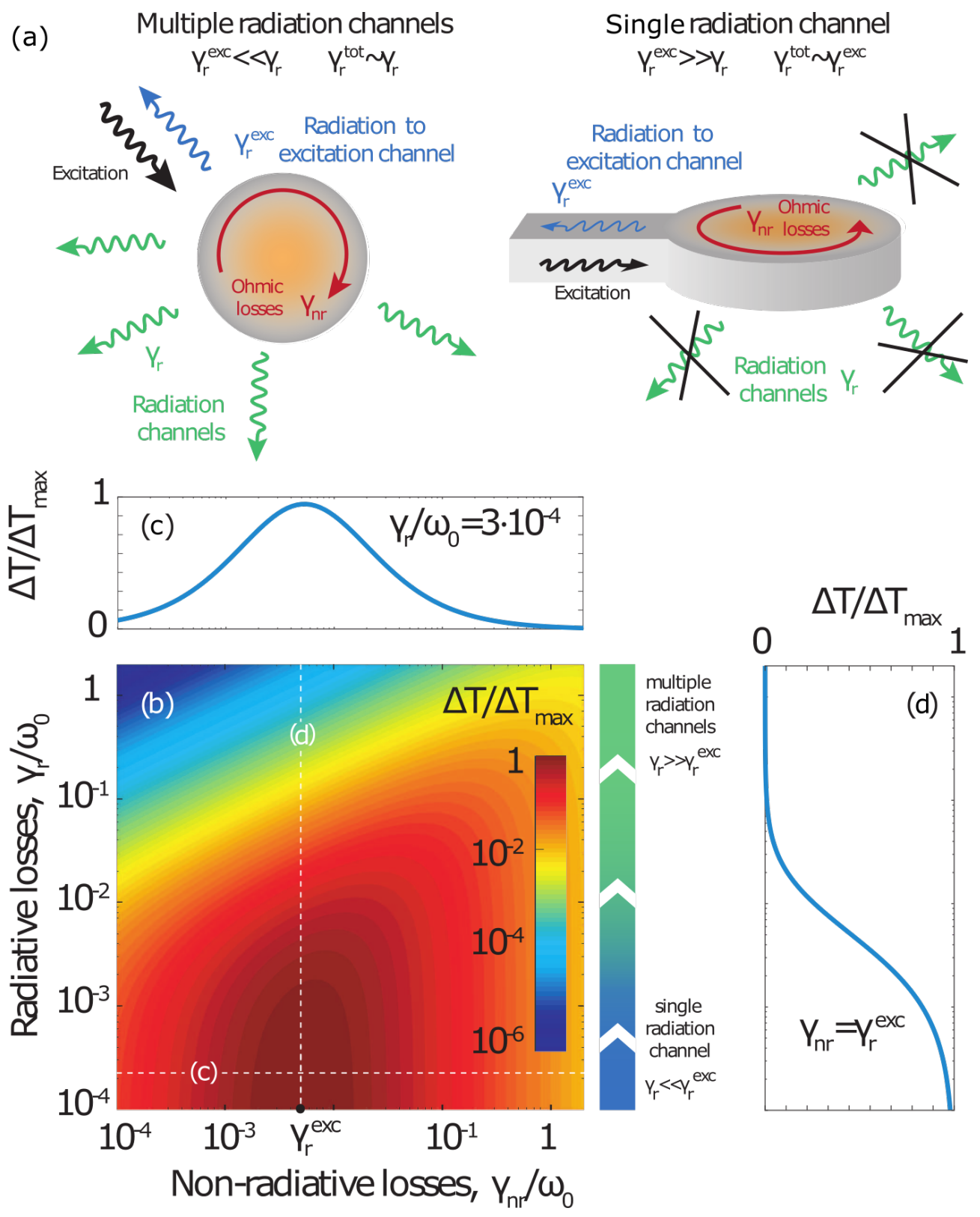

Figure 5: (a) Different excitation channels and (b) calculated losses for the optical heating optimization. The excitation channel coupling constant is taken $\Gamma_{r m} / \omega_{0}=5 \cdot 10^{-3}$. One-dimensional plots (c) and (d) correspond to cross-sections of the map along the white dash lines. 
Single radiative channel. Another important issues is that the heating efficiency is also proportional to the far-field coupling constant $\Gamma_{r m}$. Open subwavelength nanophotonic structures are usually coupled to many radiative channels, providing that $\gamma_{r}=\gamma_{r}^{\prime}+\Gamma_{r m}$ and $\gamma_{r}^{\prime} \gg \Gamma_{r m}$ (see Fig. 5). However, in particular cases the number of radiative channels can be very limited and even only one, thus making $\gamma_{r}^{\prime}=0$. In this limiting case (see Fig. 5 ) the total radiative losses are associated with the excitation channel only $\gamma_{r}=\Gamma_{r m}$ and then the maximal temperature in the critical coupling regime will be equal to $\Delta T_{\max }=|\hat{f}(\omega)|^{2} /\left(16 \pi \kappa_{s} R\right)$.

\subsection{Effect of nonradiative losses on optical heating}

The origin of optical losses in non-plasmonic materials in visible and infrared region is mainly related to the mechanism of inter-band or intraband electron absorption. While the former are related to valence-to-conductance band transitions or to excitonic transitions, the latter are connected to absorption of light by free carriers present in semiconductors.

\subsubsection{Losses in natural materials}

The results of the calculations basing on Eq. 31 are shown in Fig, 6, where the heating of dielectric and metallic nanoparticles of different sizes are compared. Namely, the dependence of temperature increase inside a nanoparticle with defined real $(\operatorname{Re}(\epsilon))$ and imaginary $(\operatorname{Im}(\epsilon))$ parts of permittivity is presented. These results give general conclusion that the relatively large dielectric nanoparticles can be heated as effective as plasmonic ones, whereas their $\operatorname{Im}(\epsilon)$ can be significantly smaller. Indeed, Figure 6 shows that low optical losses inherent for most of dielectrics do not necessarily result in weak photo-induced heating of nanoparticles.

For very small nanoparticles where $\lambda \gg D$ one can see significant optical heating in the region of negative $\operatorname{Re}(\varepsilon)$, which means only metals sup-

port heating in small structures. However, upon increasing the diameter of the nanoparticle, one can observe efficient optical heating of dielectric with even relatively low amount of optical losses $\operatorname{Im}(\varepsilon)$. If the size of the structure reaches the order of the incident wavelength and larger, optical heating mechanism becomes similar to the absorption governed by the Beer's law for bulk samples, except the cases of excitation of high-order optical modes in perfectly shaped resonators. 
From the Eq. 27) it is clear that the increasing Ohmic losses does not necessary lead to the rise of the light absorption by the nanoparticle. Basing on the performed calculations, we stress that the dielectric nanoparticles can be efficiently heated by light illumination when their radiative losses are equal to the Ohmic ones. Since dielectrics have low nonradiative losses, this condition is fulfilled for relatively big nanoparticles. Plasmonic nanoparticles, on the contrary, are expected to show the most effective heating for significantly smaller sizes [11].

\subsubsection{Doping of resonant dielectric nanoparticles}

A beneficial property of non-plasmonic materials is their ability for tuning the optical losses. Though the inter-band losses are normally provided by the band structure of the solid and hardly can be varied, the excitonic absorption can be tuned in the wide range by tuning the excitonic transition is perovskite materials [39, 38, 95]. More conventional methods of intraband absorption tuning require doping - intentional introduction of impurities into an intrinsic semiconductor for the purpose of modulating its electrical, optical and structural properties. Thus allowing effective alternation of the band structure [96, 97]. In Fig.7a the spectral dependencies of the optical absorption in c-Si and GaAs are shown for different values of the doping level. One can see that for the short wavelength region the interband indirect optical transition govern the optical losses and are almost independent on the doping level. For the photon energy below the band gap the intraband free carrier absorption becomes dominant and doping drastically increases the absorption. Despite of that in the whole spectral region of the interest the optical losses of gold prevail over the losses in the semiconductors. The doping of semiconductors also results in the change of the real part of the refractive index, which stays relatively low in the visible near-IR spectral regions. The ability to tune the losses can be efficiently used for controlling the optical heating of the nanostructure, which will be discussed in detail below.

One of the possible ways to vary nonradiative losses of a nanoresonator, thus the nonradiative losses of an optical mode, is to dope the nanoresonator material before fabrication with free carriers. Doping of a single conventional semiconductor nanoparticles is still quite a challenging task except some nanostructures based on hybrid halide perovskites, where one can achieve a drastic change in the optical and conductive properties by in-situ nanoparti-

cles doping [39]. In this regard, for the doping one should consider the films 


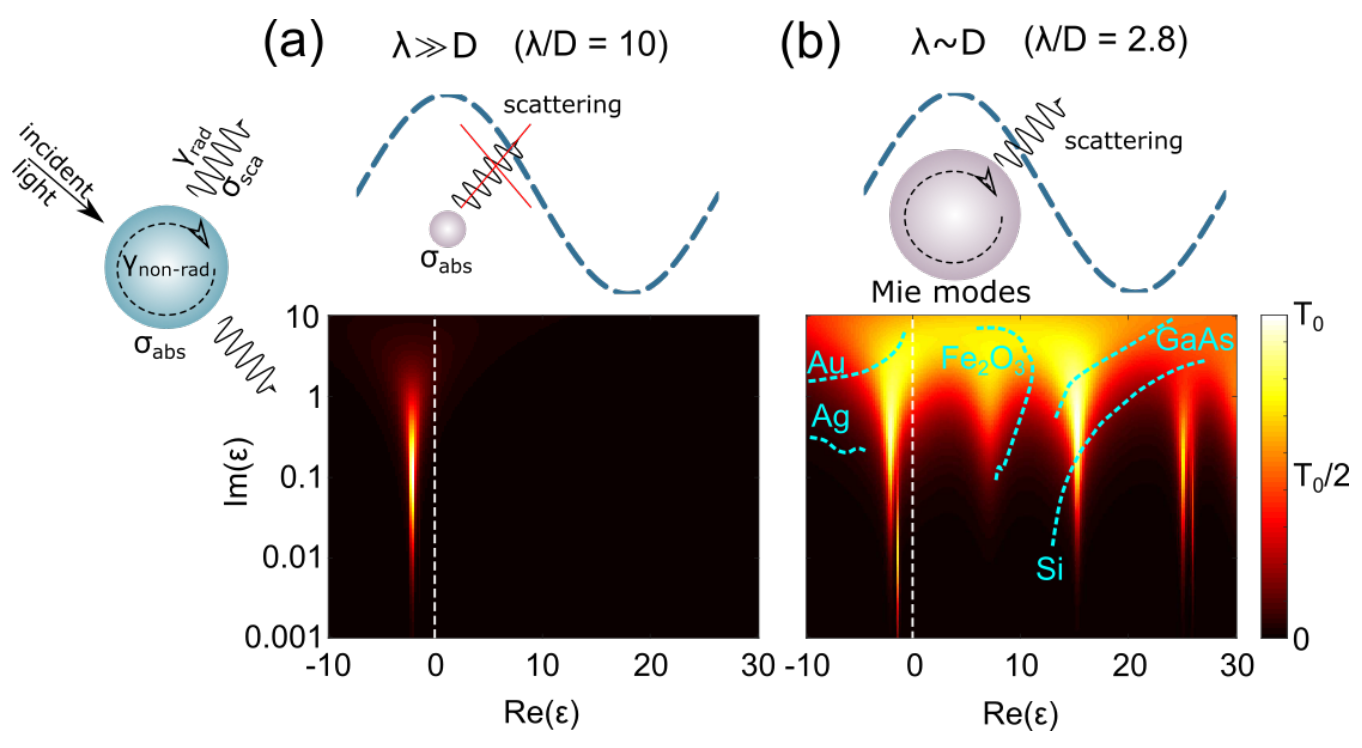

Figure 6: Effect of complex dielectric permittivity on heating. Theoretically calculated (from Mie theory) heating maps for spherical nanoparticles with deeply subwavelength (a) $(\lambda) /(D)=10$ and near-wavelength (b) $(\lambda) /(\mathrm{D})=2.8$ diameters for different real and imaginary parts of permittivity in homogeneous medium (air). Insets on top of the heating maps schematically show the size of the nanoparticle comparing to the excitation wavelength and depicts the mechanism of light-structure interaction. The scale of an imaginary part of permittivity is logarithmic. The white dashed vertical lines correspond to zero permittivity (metal-dielectric transition) and cyan dashed lines correspond to $\operatorname{Im}(\varepsilon)$ and $\operatorname{Re}(\varepsilon)$ values for given materials in the visible range. (adopted from [55]) Left inset: Schematic representation for radiative and non-radiative losses in a nanoresonator at electromagnetic wave excitation. 
which subsequently would be reshaped by lithography techniques into metasurfaces and nanoparticles. Thus, a resonant silicon nanodisk is a reasonable structure for consideration.

To start with, it is necessary to estimate the affection of doping on optical and conductive properties of the material, since it impacts significantly on optical properties [98]. The free carrier contribution to the semiconductors permittivity is described by a Drude model [99]:

$$
\begin{gathered}
\varepsilon^{\prime}=\varepsilon_{\infty}\left(1-\frac{\omega_{p}^{2} \tau^{2}}{1+\omega^{2} \tau^{2}}\right) \\
\varepsilon^{\prime \prime}=\frac{\varepsilon_{\infty} \omega_{p}^{2} \tau}{\omega\left(1+\omega^{2} \tau^{2}\right)}
\end{gathered}
$$

where the plasma frequency $\omega_{p}$ and scattering time $\tau$ are defined as $\omega_{p}=$ $\sqrt{\frac{N e^{2}}{m_{c} \varepsilon_{\infty} \varepsilon_{0}}}$ and $\tau=\frac{\mu m_{c}}{e}$, where $N$ is the free carrier concentration, $e$ is the electron charge, $m_{c}$ is the conductivity effective mass and for n-type doping of silicon $m_{c}=0.26 \cdot m_{e}, m_{e}$ is the electron mass, $\varepsilon_{0}$ and $\varepsilon_{\infty}$ are the permittivity of free space and the high frequency permittivity, respectively, and $\mu$ is the free carrier mobility.

The electron mobility and hole mobility have a similar doping dependence: for low doping concentrations the mobility is almost constant and is primarily limited by phonon scattering. At higher doping concentrations, the mobility decreases due to ionized impurity scattering with the ionized doping atoms. The actual mobility also depends on the type of dopant.

The mobility at a particular doping density is obtained from the following empiric expression:

$$
\mu=\mu_{\min }+\frac{\mu_{\max }-\mu_{\min }}{1+\left(\frac{N}{N_{r}}\right)^{\alpha}}
$$

where fitting parameters for phosphorous doping of silicon $\mu_{\min }=68.5 \mathrm{~cm}^{2} / \mathrm{V}$. $s, \mu_{\max }=1414 \mathrm{~cm}^{2} / \mathrm{V} \cdot s, N_{r}=9.2 \cdot 10^{16} \mathrm{~cm}^{-3}, \alpha=0.711$.

The mobility of the carriers and concentration affects the permittivity of the material according to the Eq. 35. Slight change of the real part of permittivity affects the spectral position and quality factor of the resonance as one can see in Fig. 7(d) where temperature of the nanosphere of 315nm radius under plane wave illumination (as schematically depicted in Fig. 7(a) is shown. The imaginary part of refractive index defines the absorption in bulk material and, therefore, optical heating. One can notice blueshift of 


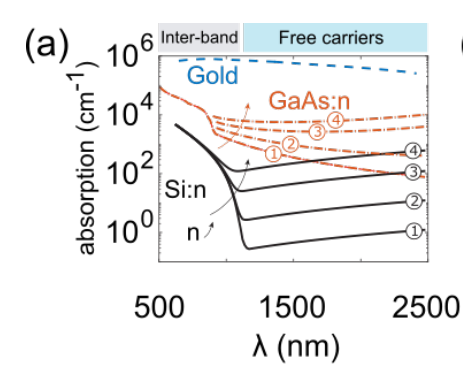

(d)

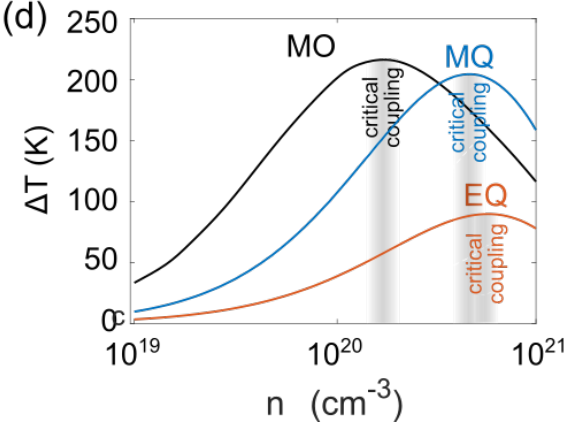

(b)
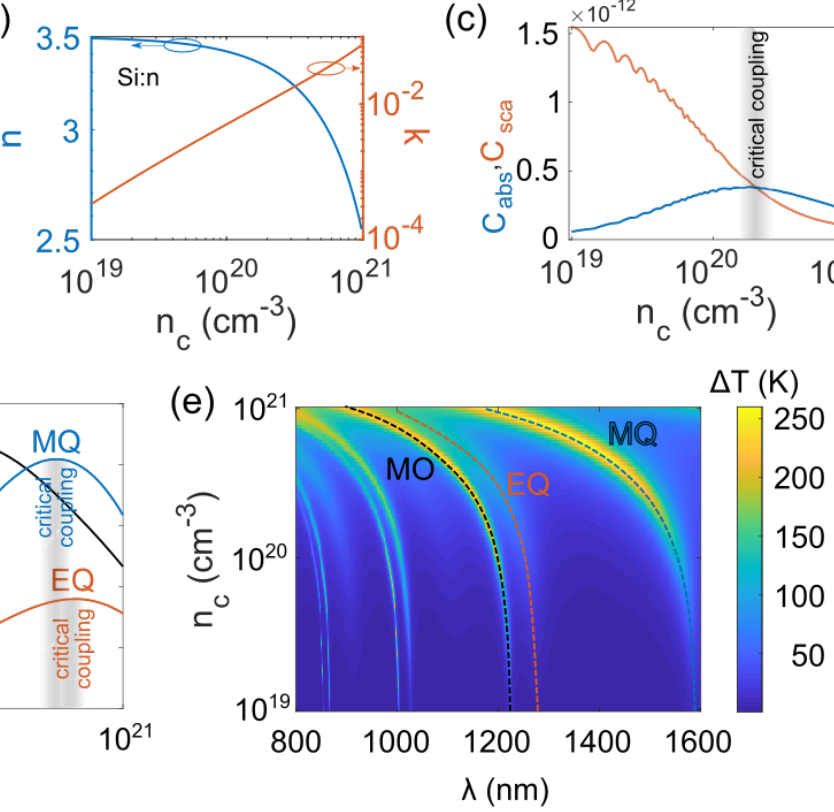

Figure 7: Loss engineering via doping. (a) Light absorption in Au, cSi:n, and GaAs:n. The absorption curves for different level of doping are shown: $1-10^{17} \mathrm{~cm}^{-3} ; 2-10^{18} \mathrm{~cm}^{-3} ; 3-10^{19} \mathrm{~cm}^{-3} ; 4-5 \cdot 10^{18} \mathrm{~cm}^{-3}$. (b) Real (blue) and imaginary (red) parts of refractive index as a function of doped free carriers concentration. (c) Maximum values of scattering (red) and absorption (blue) cross-sections in 800 to $1600 \mathrm{~nm}$ excitation wavelength at different doping levels for magnetic octupole (MO) mode only. (d) Optical heating temperature of single spherical nanoparticle with $315 \mathrm{~nm}$ radius with refractive index defined by b) and c) as a function of doping level. (e) Optical heating of spherical nanoparticle as a function of doped carriers concentration at a certain optical mode - MO, MQ, EQ - magnetic octopole, magnetic quadrupole, electric quadrupole modes, respectively.

the excited optical modes with increase of the doping level. This occurs due to decrease of the refractive index as one can see from Fig. 7(b). On the other hand, the increase of the free carriers with doping level increases dramatically the imaginary part of the refractive index, thus lowering the Q-factor of the mode, therefore spectrally broaden the resonance. However, after reaching certain level of carriers concentration, further doping does not boost optical heating, therefore it means that higher nonradiative losses does 
not necessarily lead to enhanced absorption and optical heating.

Explicitly the latter one is shown in Fig. 7(e) where optical heating at certain optical modes MO, MQ, EQ (magnetic octopole, magnetic quadrupole, electric quadrupole modes, respectively) for different levels of doping is shown. Every particular optical modes is described by its radiative losses $\gamma_{\text {rad }}$ and non-radiative, which are mostly Ohmic losses due to Joule heating $\gamma_{\text {Ohmic }}$. The latter one is defined by imaginary part of refractive index, therefore by doped carriers concentration. In general, the higher the optical mode order, the better its quality factor and lower the radiative losses $\gamma_{\text {rad }}$. Thus, the radiative and non-radiative losses balance occur at lower doping concentrations. Indeed, for higher order and higher Q-factor MO mode, the optimal optical heating is being reached at lower doping levels, whereas for MQ and EQ losses match at higher doping concentration. This result is consistent with previous predictions for plasmonic [86] and all-dielectric [87] nanoparticles described by Prof. Tribelsky and co-authors. The so-called ultimate absorption regime is being realized, where absorption matches scattering as manifested in [87], therefore the most efficient optical heating occurs at the same conditions. The Fig. 7)(c) depicts the maximum scattering cross-section and the maximum absorption cross-section of a single spherical nanoparticle of $315 \mathrm{~nm}$ radius at different values of doping for MO contribution only.

\subsubsection{Loss control via multiphoton absorption}

In nonlinear optics, it is well known effect when optical absorption becomes a function of incident light intensity [100]. In this case, several photons initiate an interband transition and generation of free carriers in the conduction band of dielectric. Once this process involves the transitions through virtual states in the band gap, it becomes considerable at relatively high light intensities. The most often case in semiconductors pumped by near-IR light is two-photon absorption (TPA), which was observed in various designs and materials at intensities $>1 \mathrm{MW} / \mathrm{cm}^{2}$. Generally, total absorption $(\alpha)$ with linear $\alpha_{0}$ and non-linear $\alpha_{N L}$ parts is written as

$$
\alpha=\alpha_{0}+\alpha_{N L}=\alpha_{0}+\beta I
$$

where $\beta$ is the two-photon absorption coefficient, which is directly connected with third-order permittivity $\chi^{(3)}$ as $\chi^{(3)}=(c \beta \varepsilon) /(8 \pi \omega), \varepsilon$ is the linear dielectric permittivity, $\omega$ is the radial frequency of light, and $c$ is the speed of light in vacuum. However, in dielectric nanoparticles, TPA should be taken 
into account in Equation 2 via free carriers generation rate written as the following terms [101]:

$$
G_{e}=\frac{\operatorname{Im}(\varepsilon)}{8 \pi \hbar}\left|E_{i n}\right|^{2}+\frac{\operatorname{Im}\left(\chi^{(3)}\right)}{16 \pi \hbar}\left|E_{i n}\right|^{4}
$$

where $E_{i n}$ is the averaged electric field over the nanoparticle volume calculated from the Mie coefficients 19, 20.

As a result, solving the Equations 1,4 together with 19,20 one can reveal the critical coupling conditions for nonlinear light absorption. Remarkably, that in the infrared range, TPA is often much stronger than linear absorption, and, thus, unexpected reaching the most optimal conditions for high quality factor resonances can result in the nanostructures damage as compared with anticipated damage threshold for bulk materials. On the other hand, such prompt processes as TPA open the new avenues for ultrafast optical switching by heating of resonant dielectric nanostructures.

\subsection{Variation of physical properties at elevated tem- peratures}

As one can see from Fig. 4, substrate materials with different thermal conductivities can dramatically affect both the heating temperature and the temperature profile. It should be noted, that upon reaching elevated temperatures, the thermal properties of materials can drastically change. Such approach is well described by Y. Li et al. [102] through macro- and microengineering of the structures and materials.

\subsection{Nonuniform near-field distribution}

Nanoantennas based on either plasmonic or all-dielectric materials can serve efficiently for electromagnetic field localization at the nanoscale, thereby can significantly enhance the field across small volumes [111, 112, 113, 114]. One should distinguish two main cases in near-field localization: external and internal relatively to the material of NPs. The first one corresponds to the localization of the field outside the nanostructure near its surface, whereas the second one accumulates energy inside the nanoobject.

The first approach is beneficial for the cases when one aims to avoid overheating of the design and provide sensing experiments with the materials deposited on the nanostructure. In this case, employing all-dielectric 


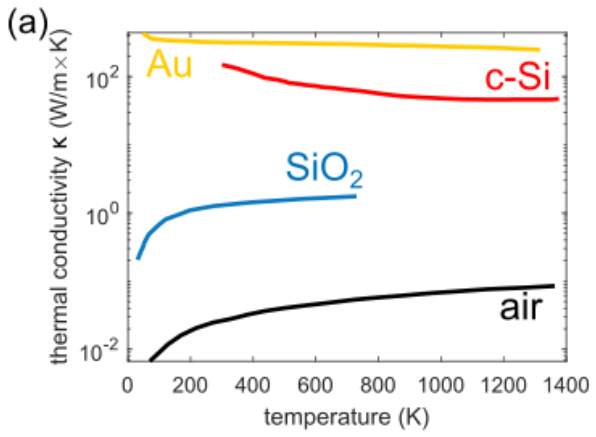

(b)

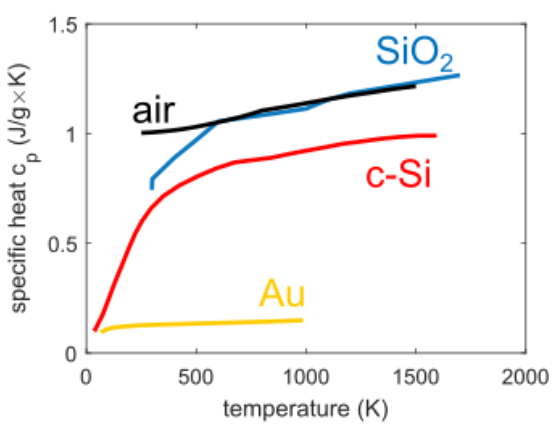

(c)

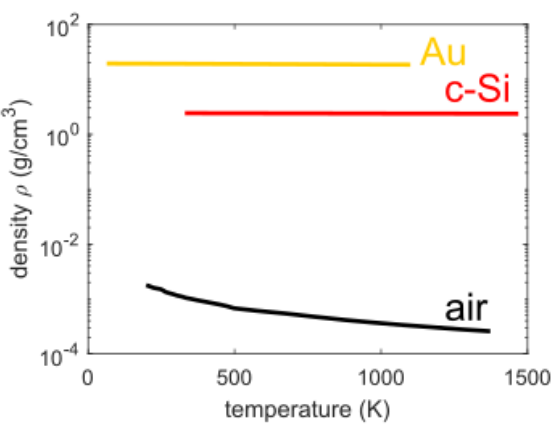

Figure 8: Thermal physical properties at elevated temperatures. (a) Thermal conductivity for $\mathrm{Au}$ [103], c-Si [104], a-SiO $\mathrm{Si}_{2}$ [105] and air [106]. (b) Specific heat for $\mathrm{Au}$ [107], c-Si [108], $\mathrm{SiO}_{2}$ [109] and air [110] . (c) Density of $\mathrm{c}-\mathrm{Si}, \mathrm{Au}$ and air at elevated temperatures.

nanophotonic designs has some advantage over metallic-based ones as shown in Fig. 9a adopted from [113. All-dielectric nanostructures with external near-field enhancement can be also designed as nanotips [115, 116, 59] or metasurfaces supporting optical modes with high quality factors [58, 61].

Designing the hot-spots inside the material of nanoparticles and initiation 
of efficient optical heating is prospective for many applications discussed in Section 5 .
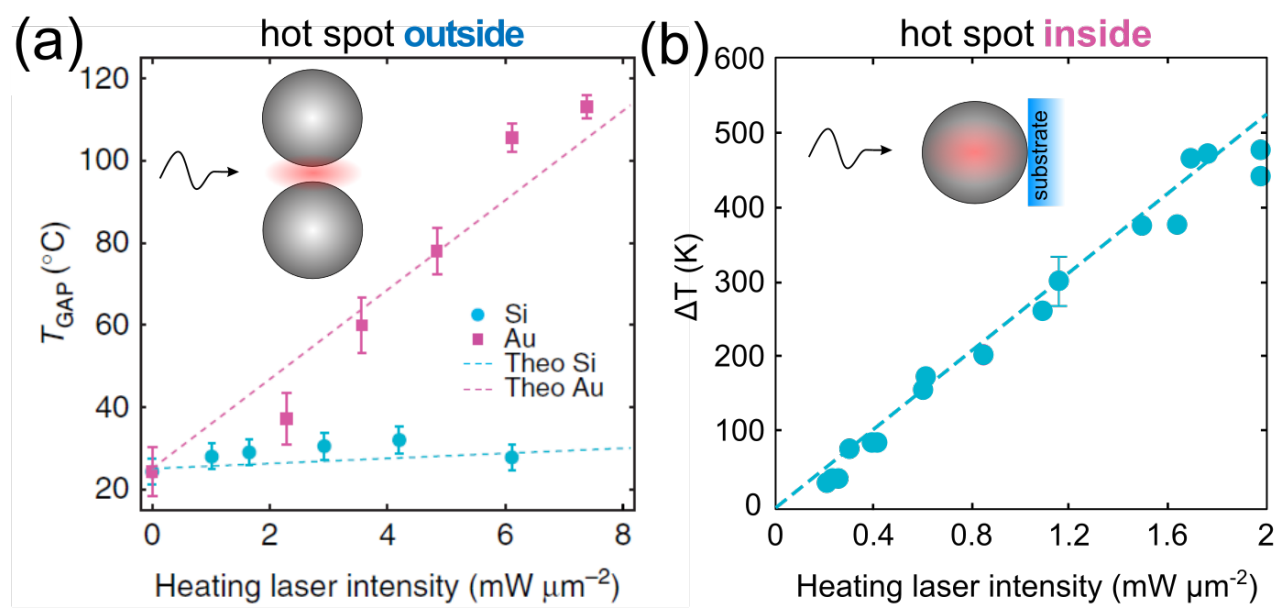

Figure 9: Cold and hot regimes of light interaction with nanoparticles. (a) Extracted temperature in the gap for selected silicon (cyan) and gold (magenta) nanoantennas as a function of the heating laser intensity at $860 \mathrm{~nm}$. The dashed lines show the numerical calculations for the temperature at the gap, presenting good agreement with the experimental data. [113]. (b) Experimental data (red circles) and numerical calculations (solid lines) for a spherical silicon nanoparticle with diameter $350 \mathrm{~nm}$ on glass. [55] 


\section{Thermometry with all-dielectric nanoanten- nas}

Measuring temperature at the nanoscale is a challenging problem in modern nanoscale science. [117, 118] Generally, an all-optical approach based on temperature-sensitive optical response of nanostructures is likely less invasive than one requiring the reading of an electrical signal, making it more suitable for uses such as measuring temperature inside a living cell of living tissue. In this regard, the all-dielectric photonics gives additional tools based on the light emission from the non-metallic material itself rather than from any additional external markers commonly utilized in thermoplasmonics.

Nanothermometry with nanoantennas can be done in different ways. As schematically shown in Fig. 10a, thermal sensitivity can arise from the material of resonant nanoparticle or from the surrounding material.

\subsection{Raman scattering}

Generally, Raman response is inelastic scattering of incident light on crystal lattice phonon, thus higher the purity and cristallinity of the nanostructure - higher the quality factor and intensity of the Raman response. The Raman scattering allow to measure temperature either to the spectral shift of the Stokes signal [53, 55] or by Stokes/anti-Stokes ratio [119].

Unlike metals, crystalline dielectrics and semiconductors possess pronounced Raman signal at room temperature, making it possible to provide direct Raman nanothermometry during optical heating even of single nanoparticle as shown in Fig,10p for the case of silicon. Indeed, the spectral position of a Raman line is known to be thermo-sensitive due to anharmonic and multiphonon-interaction effects in lattice vibrations. [53] Therefore, the frequency of an optical phonon $(\Omega)$ responsible for Raman signal is dependent on temperature as

$$
\begin{aligned}
& \Omega(T)=\Omega_{0}+A\left(1+\frac{2}{e^{x}-1}\right)+ \\
& +B\left(1+\frac{3}{e^{y}-1}+\frac{3}{\left(e^{y}-1\right)^{2}}\right),
\end{aligned}
$$

where $\Omega_{0}$ is photon frequency at zero temperature, $A$ and $B$ are constants, 
$x=\hbar \Omega_{0} / 2 k T, y=\hbar \Omega_{0} / 3 k T$, which works for crystalline silicon [53]. This function is plotted in Fig. 10c fitting experimental values calibrated with using standard heat plate. However, dielectric nanoparticles often are not monocrystalline and consist of nanoscale grains affecting (spectral shift and broadening) the Raman signal. Careful comparison of the Raman spectral shift fittings for different silicon samples based on the Eq. 40 and simpler linear approximation were carried out in the work [120], revealing considerable differences between silicon bulk wafer and nanoparticles.

The temperature resolution of Raman-based method is fundamentally limited by the spectral width of the phonon line $\left(\sim 1 \mathrm{~cm}^{-1}\right)$, and some signal post-processing could be applied to extract information on temperature more precisely.

Another Raman nanothermometry approach is based on the comparison of Stokes and anti-Stokes signal intensities. It is known that antiStokes/Stokes Raman signals ratio increases with growth of the temperature of crystallized dielectrics (e.g. silicon [121]). Because of Boltzman distribution of phonons population, an exponential dependence on temperature is observed for this ratio:

$$
\frac{I_{A}}{I_{S}}=e^{-\frac{\hbar \omega_{0}}{k T}}
$$

where $I_{A}$ and $I_{S}$ are intensities of anti-Stokes and Stokes respectively, $\hbar$ is reduced Planck constant, $\omega_{0}$ is optical phonon frequency of silicon, $k$ is Boltzmann constant and $T$ is temperature.

Remarkably, Raman signal can be strongly enhanced at optical resonances in all-dielectric designs [122, 123, 124, 125, 126]. However, this property can reduce an accuracy of such intensity-based method as Stokes/antiStokes thermometry at high temperatures, because of thermo-refractive effects changing resonant properties of dielectric nanostructures and, thus, efficiencies of Raman scattering at different wavelengths in a different manner.

\subsection{Photoluminescence}

The materials like dielectrics possessing band-gap and supporting direct interband transitions usually possess efficient photoluminescence (PL). PL process starts when material of nanostructure absorbs incident light with subsequent electron-hole pair generation, relaxation to the bottom of conduction band (or exciton formation), and further re-emission of photon with energy of the band gap (or excitonic energy). PL contains information on the emitting 

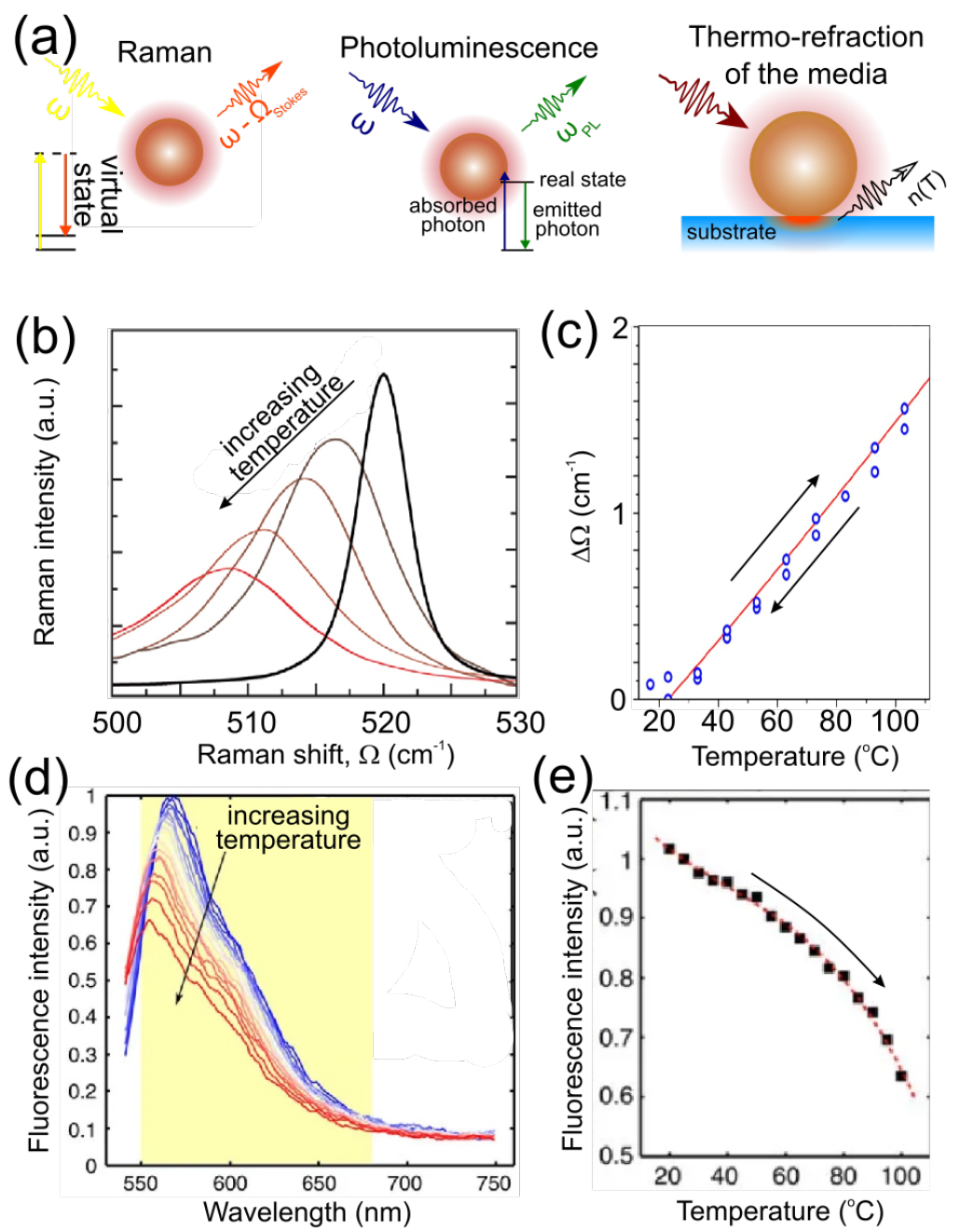

Figure 10: Nanothermometry with all-dielectric nanoantennas. (a) Schematic illustration of nanothermometry with thermally sensitive nanoparticle only (left) and with additional thermal sensitivity from surrounding material. (b) Raman spectra of c-Si NPs at different intensities [55]. (c) Connection between Raman shift and temperature as well as the fit used as a calibration curve to extract the corresponding temperature values. (d) Nile Red emission spectra taken at different temperatures. The marked zone shows the detection spectral range $(550-680 \mathrm{~nm})$. (e) The integrated intensity in the detection spectral range as well as the fit used as a calibration curve to extract the corresponding temperature values. 
material temperature, because PL spectral position $\left(\hbar \omega_{P L}\right)$, life-time $\left(\tau_{P L}\right)$, and quantum yield $\left(Q Y_{P L}\right)$ are dependent on temperature as

$$
\begin{gathered}
\hbar \omega_{P L}(T) \sim E_{g}(T)=E_{g}(0)-\frac{\xi_{1} T^{2}}{T+\xi_{2}}, \\
\frac{1}{\tau_{P L}(T)}=k_{\text {tot }}=k_{\text {rad }}+k_{\text {rad }}(T) \\
Q Y_{P L}(T)=\frac{k_{\text {rad }}}{k_{\text {tot }}}=\frac{k_{\text {rad }}}{k_{\text {rad }}+k_{\text {rad }}(T)}
\end{gathered}
$$

where $E_{g}$ is the band gap, $k_{\text {rad }}$ and $k_{\text {nrad }}$ are radiative and nonradiative recombination rates, respectively. In turn, $k_{\text {nrad }}$ is temperature-sensitive

following Arrenous-like behaviour $k_{\text {nrad }} \sim k_{\text {nrad }, 0} e^{-\delta E / k_{B} T}$, where $\delta E$ is the constant the activation energy for the nonradiative process and $k_{B}$ is the Boltzmann constant. $\xi_{1}$ and $\xi_{2}$ are material dependent constants.

Additionally, linewidth of the light-emitting system is temperature-dependent parameter. For instance, in various excitonic materials homogeneous broadening is due to scattering of the excitons by optical phonons and acoustic phonons [127]:

$$
\Gamma(T)=\sigma T+\Gamma_{L O}\left[e^{\left(E_{L O} / k_{B} T\right)}-1\right]^{-1},
$$

where $\sigma$ is the exciton-acoustic phonon coupling coefficient, $\Gamma_{L O}$ is the exciton-longitudinal optical (LO) phonon coupling coefficient, $E_{L O}$ is the LOphonon energy.

As shown in Fig. 10d,e, PL of dye enhanced by silicon nanoantennas is getting quenched exponentially with increase of temperature owing to the growth of non-radiative recombination rate in the dye molecules [113]. The achieved resolution is around few degrees. Also, thermal shift of PL line can be observed in Fig. 10d, but it can not be extracted owing to filtering of the short-wavelength wing. Thermally-sensitive PL of $\mathrm{WS}_{2}$ flakes coupled with Si nanoparticles were also employed for nanothemometry with resolution around $5-10 \mathrm{~K} .[128$

\subsection{Nonlinear scattering}

Silicon nanoparticles placed on phase changing materials (e.g. $\mathrm{VO}_{2}$ ) demonstrated considerably tunable scattering spectra owing to strong variation of 
optical properties of their surrounding media, [128] yielding temperature resolution around $1 \mathrm{~K}$. Because of high thermo-refractive coefficients for such materials as silicon, light scattering from Si nanoparticles at high temperatures can be also used for nanothermometry with high spatial resolution. [129] In contrast to plasmonic nanoparticles, which are also demonstrated thermaldependent scattering behavior due to strong increase of imaginary part of metal dielectric permittivity [130], the all-dielectric approach might have higher potential because of more degrees of freedom related to thermal tunability of far-field response caused by the interplay between magnetic and electric Mie-like resonances.

\subsection{Comparison of different nanothermometers}

Signal level and acquisition time. Efficiency of the light-emission process is one of crucial parameters because it determines the applicability of the nanothermometry based on this process. Indeed, low-efficient emission would require highly-sensitive detectors, long acquisition times, and expensive detection optical schemes. 34] Typically, photoluminescence is several orders of magnitude more efficient process as compared to Raman scattering, while elastic scattering is strongly dependent on optical contrast. However, the defect-intolerant materials can possess low PL quantum efficiency owing to high defect concentration and, thus, fast nonradiative channels of electrons relaxation. In this regard, light-emitting nanoantennas made of defect-tolerant materials can be good candidates for nanothermometry applications. 38] Moreover, achieving lasing regime in nanoantennas would accelerate radiative recombination via stimulated mechanism, making the quantum yield almost maximum. [50, 131] On the other hand, Raman scattering can be enhanced by several orders of magnitude at Mie resonances, [122, 124] making the signal acquisition time as fast as several seconds. As a result, PL, Raman, and elastic scattering from resonant all-dielectric nanostructures can give comparable seconds-level signal strongly dependent on various conditions.

Sensitivity. Sensitivity is an absolute quantity: it specifies the smallest, absolute amount of change that can be detected by the sensor. in general, different nanothermometry techniques can be compared using the relative sensitivity, defined as 132

$$
S_{r}=\frac{d X / d T}{X}
$$


which allows for standardizing the various methods regardless of the difference in underlying working principle and measured observable, $X$. According to this definition, the sensitivity is expressed in $\% \mathrm{~K}^{-1}$ units. Typical values of the relative sensitivity are on the level of $0.1-10 \% \mathrm{~K}^{-1}$ for PL-based nanothermometers around room-temperature. In turn, Raman-based techniques exhibit $\mathrm{S}$ values around $0.01-0.1 \% \mathrm{~K}^{-1}$ as shown in Figure 11 .

Temperature range, robustness, and stability. One of the main shortcomings of thermosensing techniques based on organic dyes is their limited operating range - a few tens of degrees around room temperature. For example, dye molecules integrated with a resonant silicon dimer were burned at relatively low temperatures. [113] Record-high temperature range up to $\Delta \mathrm{T}=750 \mathrm{~K}$ for PL-based nanothermometers was demonstrated in a specific nanomaterial based on oxide NPs doped with rare earth and covered by Au NPs [32]. In turn, silicon nanoparticles were successfully tested in the range of $\mathrm{T}=300-1000 \mathrm{~K}$ [55, 133, 134, 120, 135], where any thermally-sensitive organics would die or PL would vanish because of exponentially increased nonradiative losses. Also, Raman-emitting approaches are more stable and robust in general, because they are not affected by any additional FRETbased or oxygen-based channels of nonradiative recombination during the measurement process.

\section{Applications of all-dielectric thermonanopho- tonics}

The described in previous sections physical phenomena can be employed in variety of applications which are illustrated in Fig. 12. The applications will appear according to the enhancement of temperature from low to high, which is generally correlated with the range of light sources irradiating the nanostructures: from broadband solar light to highly intensive laser radiation.

\subsection{Basic properties of materials employed for ther- monanophotonics}

The main physical parameters of bulk dielectric materials shown in Table 2 play key role in the relation to real applications of thermo-photonics. Indeed, according to the above mentioned theoretical models, the knowledge 


\begin{tabular}{|c|c|c|c|}
\hline & Materials for nanothermometry & $\mathrm{S}_{r},\left[\mathrm{~K}^{-1}\right]$ at $300 \mathrm{~K}$ & $\Delta \mathrm{T}[\mathrm{K}]$ \\
\hline \multirow[t]{5}{*}{ Organic dyes (ODs) } & Ruphen (PL intensity) 136 & 0.0093 & $280-315$ \\
\hline & Bis (pyrene) propane (PL ratio) 137 & 0.387 & $310-465$ \\
\hline & Rhodamine-B (PL Intensity) 138] & 0.02 & $287-363$ \\
\hline & Fluorescein (PL anisotropy) 139] & 0.057 & 293-352 \\
\hline & Triarylboron (wavelength shift) 140 & 0.005 & $223-373$ \\
\hline \multirow[t]{5}{*}{ Quantum dots (QDs) } & CdSe (wavelength shift) 141 & $1.61 \times 10^{-4}$ & $293-323$ \\
\hline & $\mathrm{CdSe} / \mathrm{ZnS}$ (PL intensity) 51] & 0.019 & 278-313 \\
\hline & CdSe/Zns (wavelength shift) 142] & $2.67 \times 10^{-4}$ & $284-320$ \\
\hline & $\mathrm{Zn}_{1-x} \mathrm{Mn}_{x} \mathrm{Se} / \mathrm{ZnCdSe}$ (PL ratio) 143] & 0.018 & $134-400$ \\
\hline & CdTe (PL lifetime) 144 & 0.008 & 293-333 \\
\hline \multirow[t]{7}{*}{ Up-conversion nanoparticles (UCNPs) } & $\mathrm{Er}^{3+} / \mathrm{Yb}^{3+} \mathrm{CaF}_{2}$ (PL ratio) 145 & 0.015 & $293-318$ \\
\hline & $\mathrm{Tm}^{3+} / \mathrm{Yb}^{3+} \mathrm{CaF}_{2}$ (PL ratio) 145 & 0.002 & 293-318 \\
\hline & $\mathrm{NaYF}_{4}: \mathrm{Er}^{3+}, \mathrm{Yb}^{3+}$ (PL ratio) 146 & 0.0114 & $298-334$ \\
\hline & $\mathrm{NaLuF}_{4}: \mathrm{Yb}, \mathrm{Er}$ 147] & 0.009 & $273-348$ \\
\hline & $\mathrm{ZnO}: \mathrm{Er}^{3+}$ (PL ratio) 148 & 0.0098 & $278-463$ \\
\hline & $\beta-\mathrm{NaYF}_{4}: 20 \% \mathrm{Yb}_{2} \% \operatorname{Er}(\mathrm{PL}$ ratio) 149 & 0.0157 & 294-334 \\
\hline & $(\mathrm{Gd}, \mathrm{Yb}, \mathrm{Er})_{2} \mathrm{O}_{3}$ (PL ratio) 32 & 0.017 & $300-1050$ \\
\hline \multirow[t]{5}{*}{ Nanodiamonds (NDs) } & NV (PL intensity) 150] & 0.01 & $295-400$ \\
\hline & $\mathrm{GeV}$ (ZPL linewidth) 151] & 0.0064 & $150-400$ \\
\hline & $\operatorname{SiV}$ (ZPL shift) [152] & $1.61 \times 10^{-5}$ & $285-305$ \\
\hline & SnV (ZPL shift) 153] & $8.66 \times 10^{-5}$ & 295-315 \\
\hline & $\mathrm{GeV}$ (anti-Stokes) [154] & 0.014 & $150-400$ \\
\hline \multirow[t]{3}{*}{ Optically resonant nanoparticles (RNPs) } & Au nanorod (anti-Stokes PL) 34] & $10^{-3}$ & $300-1300[155$ \\
\hline & Si (Stokes Raman shift) [55] & $2 \times 10^{-4}$ & $0-1685$ 156. 157. 158 \\
\hline & $\alpha-\mathrm{Fe}_{2} \mathrm{O}_{3}$ (Stokes Raman shift) 159 & $4 \times 10^{-4}$ & $0-1840 \quad 160$ \\
\hline
\end{tabular}

Table 1: Nanothermometers. List of materials and nanothermometry techniques based on light-emitting materials, nanostructures, and nanomaterials. Adopted from [118] and extended by resonant plasmonic and non-plasmonic nanoparticles. 


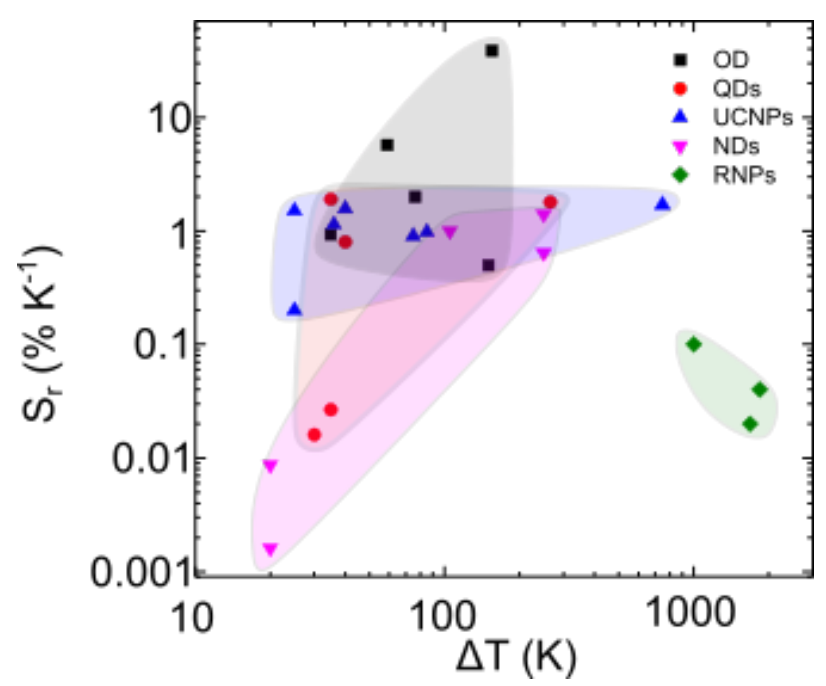

Figure 11: Comparison of nanoscale thermometers. Experimental parameters relative sensitivity $S_{r}$ and working temperatures range $\Delta \mathrm{T}$ ) for the known nanothermometers summarized in Table 1 (dots). Organic dyes (OD, black), quantum dots (QDs, red), upconversion nanoparticles (UCNPs), nanodiamonds (NDs), resonant nanoparticles (RNPs).

of thermal conductivity, thermal capacity and band gap determine the heating efficiency in the given spectral range, while thermo-optical coefficient and thermal expansion coefficient are important for additional nonlinear feedback of the heated nanostructure.

\subsection{Optical cooling}

In 1929, it was suggested that solids could cool through anti-Stokes fluorescence in which a substance absorbs a photon and then emits one of greater energy [185] (so-called up-conversion) via absorption of an additional phonon for energy conservation (see Fig. 13 a).

For decades the advantages of laser cooling of solids were connected with RE-doped glasses and crystals. In 1950, it was proposed the use of rareearth ions in transparent solids as a fluorescent cooler because of their high quantum efficiencies and narrow spectral lines [186]. The main advantage of $\mathrm{RE}$ ions is the optically active $4 f$ electrons shielded by the filled $5 \mathrm{~s}$ and $5 p$ outer shells, which limit interaction with the lattice surrounding the RE ion 


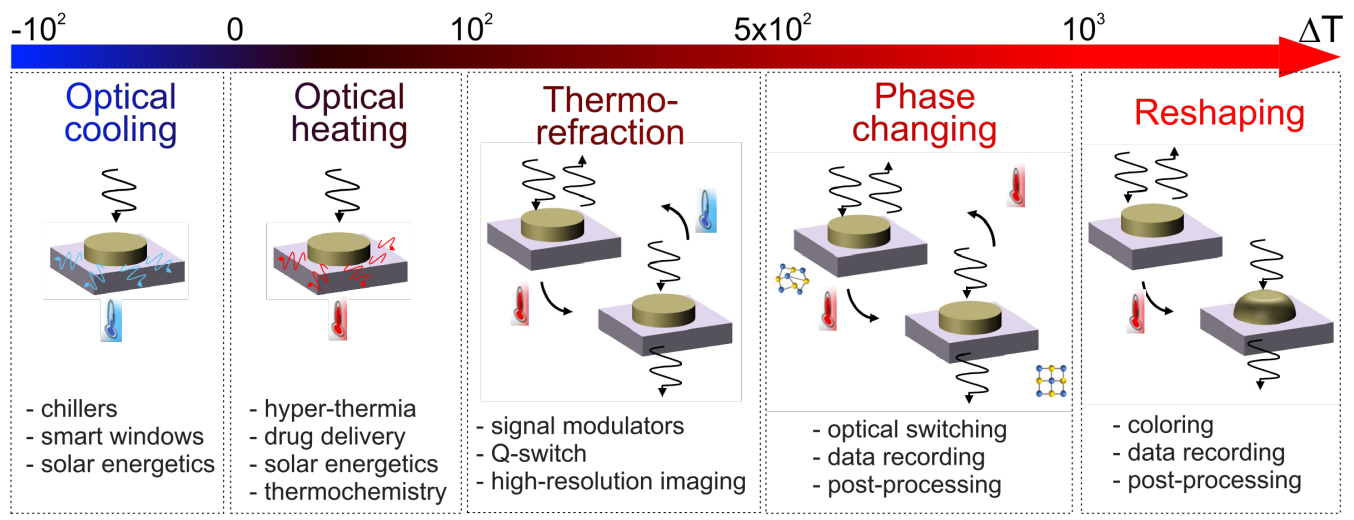

Figure 12: Applications of thermally-induced processes. Conceptual images of different thermally-induced processes presented on the temperature scale, from lower to higher.

\begin{tabular}{|c|c|c|c|c|c|c|}
\hline \multirow{2}{*}{$\begin{array}{c}\text { Material } \\
\text { (Crystalline state) }\end{array}$} & $\begin{array}{c}\text { Melting } \\
\text { Temperature }\end{array}$ & $\begin{array}{c}\text { Heat } \\
\text { Capacity }\end{array}$ & $\begin{array}{c}\text { Thermal } \\
\text { Conductivity }\end{array}$ & $\begin{array}{l}\text { Thermo-optical } \\
\text { coefficient }\end{array}$ & $\begin{array}{c}\text { Thermal expansion } \\
\text { coefficient }\end{array}$ & Band gap \\
\hline & $\mathrm{K}$ & $\mathrm{J} /(\mathrm{g} \mathrm{K})$ & $\mathrm{W} /(\mathrm{m} \mathrm{K})$ & $10^{-6} \mathrm{~K}^{-1}$ & $10^{-6} \mathrm{~K}^{-1}$ & $\mathrm{eV}$ \\
\hline $\mathrm{Si}(\mathrm{c})$ & 1687 161] & 0.713161 & 156161 & $\begin{array}{l}250(>2 \mu \mathrm{m}) \\
150(>2 \mu \mathrm{m})\end{array}$ & $2.616 \square 161$ & 1.14(i) 163 \\
\hline $\mathrm{Si}$ (a) & 1400164 & $21 \mathrm{~J} / \mathrm{mol} / \mathrm{K}$ & 1.8165 & $\begin{array}{l}-147+485 \mathrm{i}(@ 633 \mathrm{~nm}) \\
271+225 \mathrm{i}(@ 752 \mathrm{~nm}) \text { 166 } \\
\quad \text { +imaginary part }\end{array}$ & $\sim 1$ & 1.14(i) 163 \\
\hline $\mathrm{Ge}$ & 1210.4161 & 0.3295161 & $60 \square 1$ & $\begin{array}{c}400(>2 \mu \mathrm{m}) \\
400-500(>2 \mu \mathrm{m})\end{array}$ & 5.75161 & $0.67(\mathrm{i}) 163$ \\
\hline GaAs & $1513[161]$ & $0.327[161]$ & $45[161]$ & $250(@ 1.15 \mu \mathrm{m})[162]$ & $6.03[161]$ & $1.43(\mathrm{~d})[163$ \\
\hline $\mathrm{GaP}$ & $1730[161]$ & $0.313[161$ & $77[161$ & $160(@ 0.63 \mu \mathrm{m}) \quad 162$ & 4.89161 & 2.26 (d) 163 \\
\hline CdTe & 1365 161] & $0.211[161$ & $7.5[161$ & $147(@ 1.15 \mu \mathrm{m})$ 162 & $4.7[161]$ & 1.49 (d) 163 \\
\hline $\mathrm{PbTe}$ & 1197 & $0.0031[168]$ & $1.98[168]$ & $-1.4 \times 10^{-3}(>6 \mu \mathrm{m})[169]$ & $19.8[170]$ & $0.32(\mathrm{~d}) \quad 170$ \\
\hline $\mathrm{Ge}_{2} \mathrm{Sb}_{2} \mathrm{Te}_{5}(\mathrm{a}, \mathrm{c})$ & $\begin{array}{c}900 \\
(\mathrm{a}->\mathrm{c} \sim 400)\end{array}$ & 0.212173 & $\begin{array}{c}0.19, \\
0.57-1.5\end{array}$ & $\begin{array}{c}\text { a: } \Delta \mathrm{k}=11.7 \\
\Delta \mathrm{n}=35 \\
\text { c: } \Delta \mathrm{k}=113 \\
\Delta \mathrm{n}=-65(@ 1.55 \mu \mathrm{m})\end{array}$ & $2-7 \quad 176$ & $0.95(d) \quad 163$ \\
\hline $\mathrm{MAPbCl}_{3}$ & $650 \quad[177]$ & $0.492[178]$ & $0.46[178]$ & $-300[178$ & 30 [179] & $3.15[178]$ \\
\hline $\mathrm{Au}$ & $1337 \quad 76$ & 0.129 76] & $318[76]$ & $-300--700[180$ & $14[181]$ & \\
\hline $\mathrm{Ag}$ & $\begin{array}{ll}1235 & 76 \\
\end{array}$ & $0.24[76]$ & 42976 & $-300--700[182$ & $18.9[181]$ & Metals \\
\hline $\mathrm{Al}$ & $933[76]$ & $0.9[76]$ & $237[76]$ & $\mathrm{C}_{T R}=114(@ 780 \mathrm{~nm})$ & $23.3[181]$ & \\
\hline
\end{tabular}

Table 2: Properties of the materials usually used for thermophotonic applications. Parameters are taken at ambient conditions if not noted otherwise. Indices in brackets for band gap denote direct (d) or indirect (i) band. Indices in brackets for material names are denoted crystalline phase - amorphous (a) or crystalline (c). $\mathrm{C}_{T R}$ stands for thermoreflectance coefficient. 
(a)

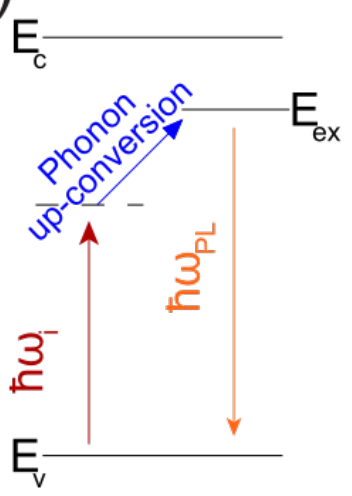

(c)

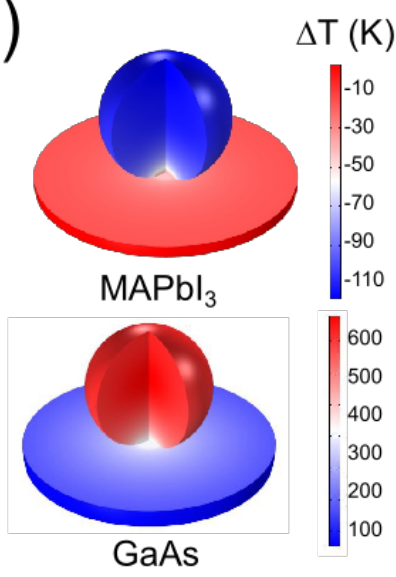

(b)

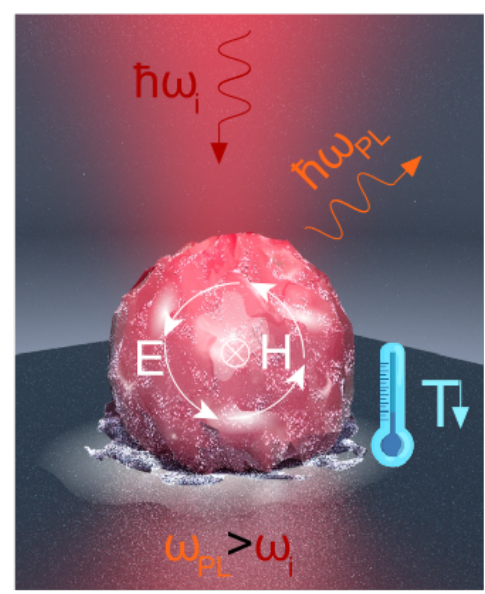

(d)

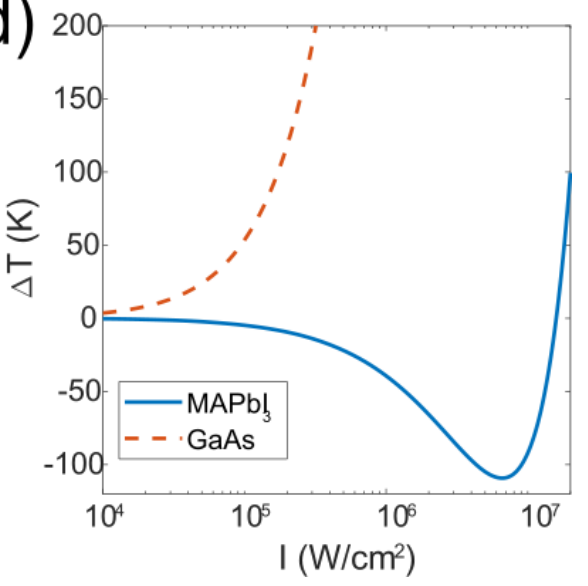

Figure 13: Optical cooling. (a) Scheme of photoluminescence upconversion mechanism. (b) Sketch of optical cooling via enhanced, upon excitation of Mie-modes, upconversion photoluminescence. (c) Temperature distributions for $530 \mathrm{~nm} \mathrm{MAPbI}{ }_{3} \mathrm{NP}$ under $980 \mathrm{~nm}$ laser illumination with $7 \cdot 10^{6} \mathrm{~W} / \mathrm{cm}^{2}$ intensity and $340 \mathrm{~nm}$ GaAs NP under laser illumination with the same wavelength and $10^{6} \mathrm{~W} / \mathrm{cm}^{2}$ intensity. (d) The dependence of temperature change for $530 \mathrm{~nm} \mathrm{MAPbI}_{3} \mathrm{NP}$ (blue solid line) and $340 \mathrm{~nm}$ GaAs NP (red dashed line) on substrate on laser intensity at wavelength $\lambda=980 \mathrm{~nm}$. [184] 
and suppress nonradiative decay. The successful realization of laser cooling of rare-earth-doped solids was demonstrated in 1995 [187]. Since then, laserinduced cooling has been observed in a wide variety of glasses and crystals doped with ytterbium $\left(\mathrm{Yb}^{3+}\right)$, thulium $\left(\mathrm{Tm}^{3+}\right)$ and erbium $\left(\mathrm{Er}^{3+}\right)$. [188, 189. Among the various dielectric materials, low trap-state density, high PL quantum yield, as well as pronounced excitonic states at room temperature in bulk halide perovskites resulted in high efficiency of up-conversion, and, thus, in the decrease of local temperature by $20 \mathrm{~K}$ upon laser irradiation [52].

There are two ways to enhance optical cooling as it follows from Eq. 47. Firstly, one can increase external radiative quantum efficiency via decreasing the radiation lifetime. Secondly, one can decrease frequency of absorbed light. Balance of these two effects determines the cooling efficiency, which can be expressed by the ratio introduced in Ref. [190] as following:

$$
\eta_{c}=\frac{P_{P L}}{P_{P L}+P_{a b s}}=\eta \frac{\omega_{P L}}{\omega_{i}}-1
$$

where $\eta$ is luminescence quantum efficiency, $\omega_{P L}$ is $\mathrm{PL}$ frequency, $P_{P L}\left(P_{a b s}\right)$ is the emitted (absorbed) power of light.

In order to determine the temperature change of the NP under laser illumination, one should consider the stationary heat transfer equation and take into account only radial heat distribution:

$$
\nabla(\kappa \nabla T)=\eta_{c} \frac{I \sigma_{a b s}}{V}
$$

where $\kappa$ is thermal conductivity.

Further optimization of the optical cooling requires both enhanced quantum yield of emission and improved absorption of incident light in the material. Recently, it was proposed that the resonant phonon-assisted upconversion photoluminescence optical cooling approach can be optimized via enhancement of the emission rate and photoexcitation with Mie resonances in nanoparticles at pump and emission wavelengths (see schematic illustration in Fig. 13b). [184] In this case, the expression for the temperature variance inside the NP can be found by solving the equation for thermal diffusion and appears to be following:

$$
\Delta T=-\eta_{c} \frac{\sigma_{a b s} I}{2 \pi \kappa D},
$$


where $\kappa$ is the thermal conductivity of the surrounding medium and $D$ is the diameter of a nanoscale sphere. Remarkably, that $\eta_{c}$ is directly connected with Purcell effect and, thus, cooling efficiency can be enhanced when emission spectra is overlapped with a resonant mode in the nanoparticle.

The numerical and analytical modeling revealed that the highest cooling efficiencies for a halide perovskite spherical NP correspond to the excitation of magnetic-type Mie modes. [184] Namely, magnetic octupole at the emission and magnetic quadrupole at absorption allow for cooling a single nanocavity by $\Delta T \approx-110 \mathrm{~K}$ at realistic conditions. In opposite, GaAs nanoparticles with less efficient PL can not be optically cooled with this mechanism as shown in Fig. 13r, d.

\subsection{Solar energy and heat conversion}

In our everyday life, the lowest irradiation intensities capable to heat various objects are coming from the Sun, i.e. around $100 \mathrm{~W} / \mathrm{m}^{2}$. A typical sunlight application is a solar water heater placed usually on a roof to collect solar energy. Meanwhile, many other applications recently appeared for photothermal devices based on solar energy: water desalination, surface sterilization, deicing, water evaporators, etc. [191, 192]. One of the typical design is to use separate sunlight absorbers (some black surfaces), which convert solar energy into thermal energy that is then transferred to water. However, a more efficient way of water heating is heating through nanoparticles dispersed in water, which is called nanofluid [193]. In this case, absorbing and generating heat nanoparticles transfers it directly to the water. Thus, the main challenge here is to provide efficient sunlight harvesting in as broad spectral range as possible and high light-to-heat conversion efficiency.

Resonant nanoparticles made of semiconductor with indirect band gap absorption (like Si [194], Ge [195], and Te [196]) are the best candidates for such spectrally broadband light energy conversion to heat. Indeed sunlight on the Earth surface is quite spectrally broadband $(\lambda \approx 300-2000 \mathrm{~nm})$ source of energy. An example of water evaporation upon sunlight illumination is shown in Fig. 14. Figure 14f depicts the evolution of water evaporation at different Si nanoparticles concentrations [194]. The ability of Si NPs for broadband light absorption with subsequent conversion to thermal energy by

means of Mie-resonances results in high light energy conversion efficiency of the nanofluid (up to 55\%), which is the sum of energies consumed to vaporize 
$E_{v}$ water and heat $E_{h}$ water divided to the energy of incident light $E_{i n}$ :

$$
\eta_{\text {heat }}=\frac{E_{v}+E_{h}}{E_{\text {in }}}
$$

The light energy conversion efficiencies for various types of nanofluids based on Mie-resonant NPs are summarized in Table 3

\begin{tabular}{|l|l|l|}
\hline nanofluid type & $\eta_{\text {heat }}$ & ref. \\
\hline water & $29 \%$ & {$[\mathbf{1 9 4}$} \\
\hline Si NPs@water & $55 \%$ & {$[194$} \\
\hline Ge NPs@water & $67 \%$ & {$[195]$} \\
\hline Te NPs@water & $85 \%$ & {$[196$} \\
\hline
\end{tabular}

Table 3: Energy conversion efficiencies for various types of nanofluids upon sunlight illumination.

Generally, Si and Ge NPs are non-toxic materials, and can be used in liquids for many of real-life applications. Although tellurium NPs were also utilized for efficient water heating upon sunlight illumination [196], tellurium is considered to be toxic. However, it plays an important role in many biological systems, while the content of tellurium in the human body is more than $0.5 \mathrm{~g}$, being the fourth most abundant trace element after Fe, Zn, and $\mathrm{Rb}$ in the human body and it is unusually abundant in human food and plants [197]. Further progress in this field can be done with hybrid metaldielectric NPs, where Mie-like and plasmonic resonances coupling results in high light energy conversion efficiencies (e.g. with $\mathrm{TiO}_{2} / \mathrm{Au}$ NPs [198]).

\subsection{Biomedical applications}

All-dielectric resonant nanostructures are widely used for optical sensing of various bio-objects like proteins, antibodies, and related molecules [199, 58], where the role of Mie resonances is crucial [60]. Despite thermal effects are ignored in the most of works on this topic, temperature growth can be considerable if the nanostructures absorb high enough power of the incident light.

According to Sections 3 and 4, once local temperature can be measured directly, two main regimes of light interaction with all-dielectric nanoresonators can be distinguished. Fig. 9 shows experimental data for the cases 

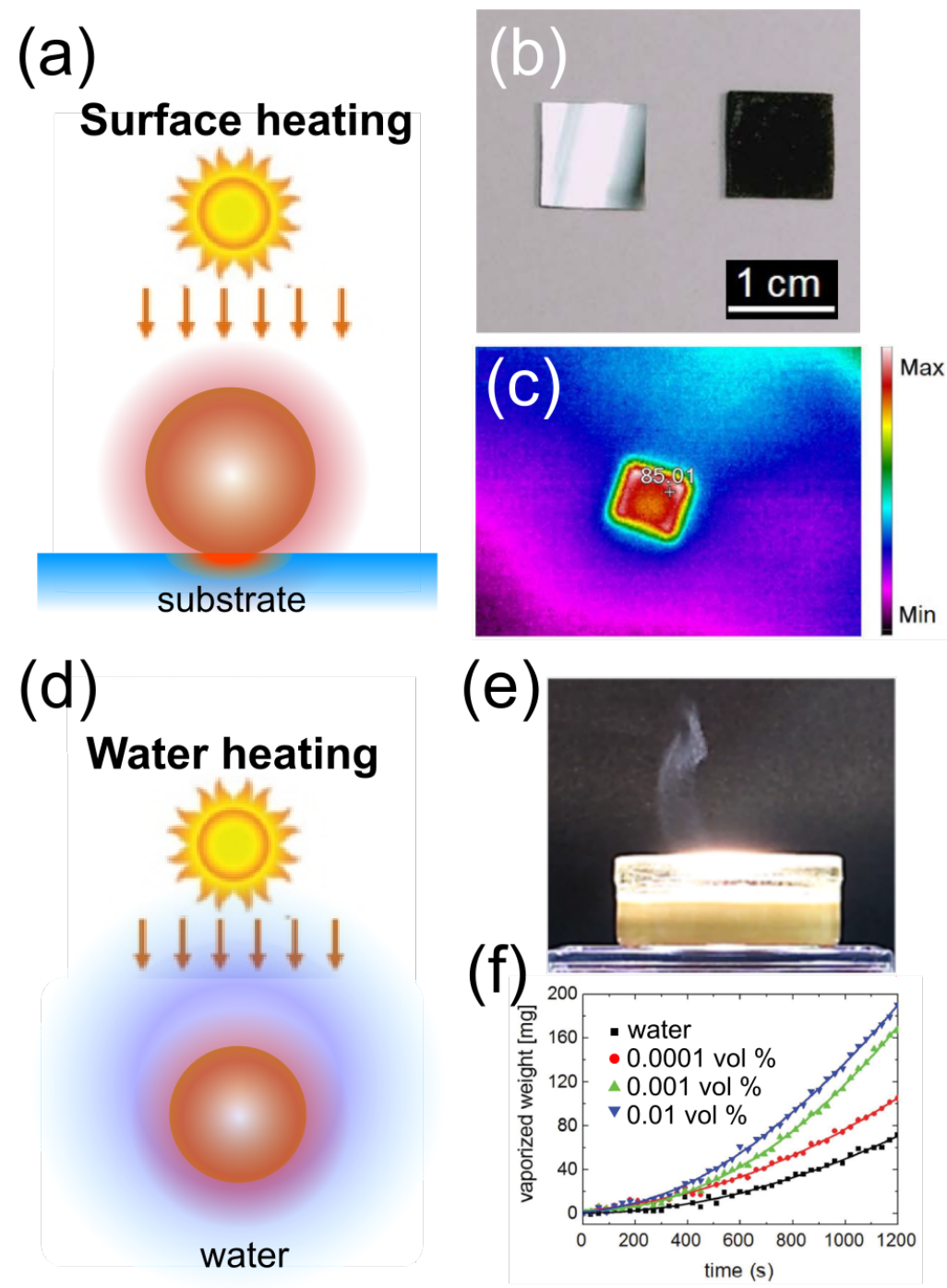

Figure 14: Solar energy applications. (a) Schematic diagram of a nanoparticle on surface irradiated by sunlight. (b) Photograph of a bare Si wafer (left) and Te nanoparticle layer deposited on Si substrate (right). (c) Steady-state thermal image of the Te nanoparticle absorber. [196] (d) Schematic diagram of a nanoparticle in water irradiated by sunlight. (e) The photo of $0.1 \mathrm{vol} \%$ Si nanofluid at the irradiation of $800 \mathrm{~mW} / \mathrm{cm}^{2}$ from the solar simulator [194] (e) Vaporized weight of the water under the illumination of simulated sunlight at $80 \mathrm{~mW} / \mathrm{cm}^{2}$. The concentration of Si nanoparticles in nanofluid is varied from 0 to 0.01 vol $\%$ where 0 vol\% means pure water [195]. 
when two optically coupled silicon nanoparticles are not heated upon highintense laser illumination (Fig. 9a), and strongly heated a single resonant silicon nanoparticle at much lower intensity (Fig. 9b). The reason of such big difference lies in the amount of light energy accumulated inside and outside the nanoresonators. Indeed, the silicon nanodimer shown in the work [113] is an optimal design for PL and SERS in the cold regime when parasitic heating is diminished, because hot spot is concentrated in the gap between nanoparticles, while much less energy accumulated in the silicon parts. However, once nanoparticles are coupled with light resonantly, the opposite situation can be observed when the nanoparticle undergoes strong heating, which was shown experimentally [55].

Similar transition between 'cold' and 'hot' regimes of interaction was realized for a silicon nanoparticle on gold [134, behaving like a dimer because of formation of 'mirror image' modes [200]. Moreover, this design is prospective for sensing applications [201, 202] owing to high near-field concentration in the gap between a nanoparticle and a metal. First SERS measurements in the gap between a resonant dielectric nanoparticle and metal was carried out by Huang et al. [203] and showed up to $10^{7}$ Raman signal enhancement. The advantage of existence of thermally sensitive part in this design is in the ability to study in situ local Raman response of the material (vibrational strength and spectral changes) in the gap between nanoparticle and metal with simultaneous temperature control in broad range [134]. Controllable local heating and modification of surrounding material with all-dielectric nanoantennas was also employed for intracellular optical opening of microcapsules for drug delivery applications [159].

Despite the ability of dielectric resonant nanostructures to be heated efficiently by light in the visible range, biological applications related to IR sources are not affected by any thermal effects, and can be safely employed [58, 61].

\subsection{Thermorefractive optical nonlinearities}

With the increase of the temperature the optical constants of the solids can not be generally considered as constant and start to depend on temperature, which is referred to as thermo-optical effect. There are several physical mechanisms lying in the origin of this effect. One of the most important is related to the dependence of the electronic band gap on the temperature [207] due to thermal expansion of lattice. That, along with the temperature dependent 

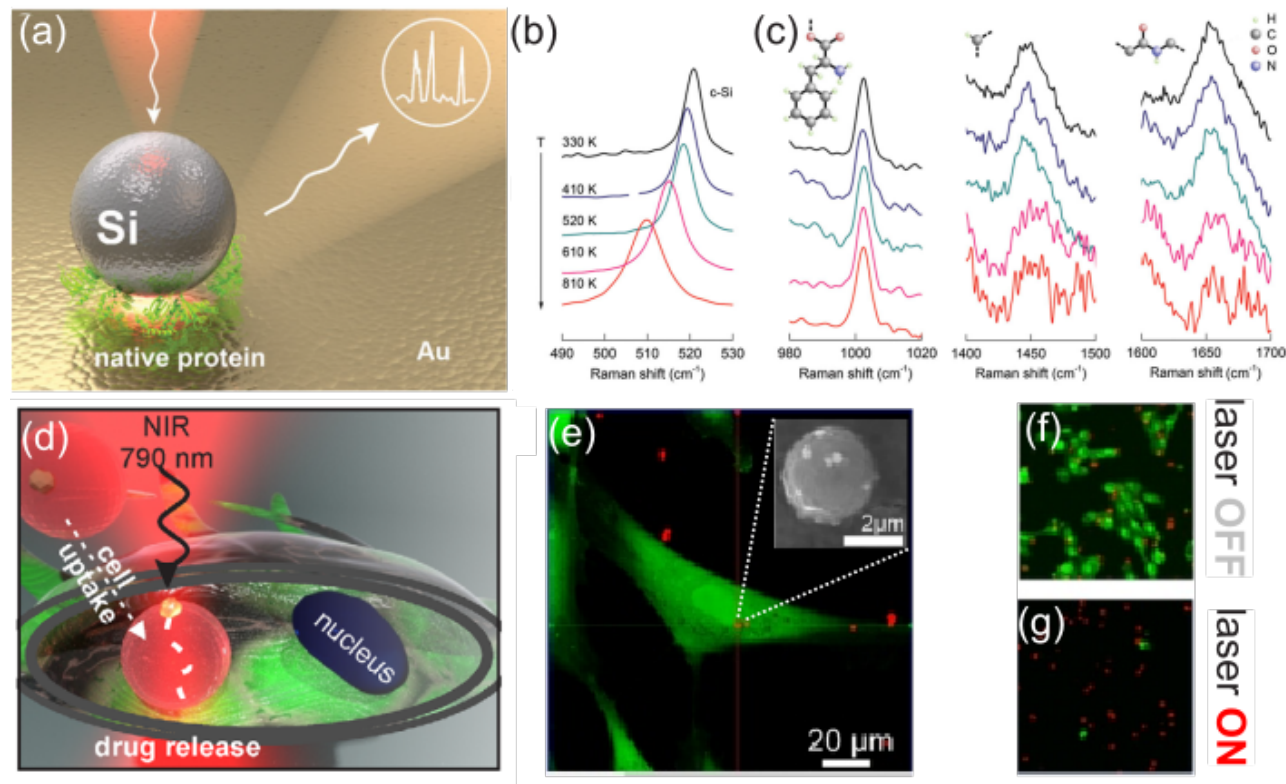

Figure 15: Biomedical applications. (a) Schematic illustration of the hybrid nanocavity for simultaneous molecular sensing, nanoscale thermometry, and tracing the heat-induced events through the change in Raman scattering signal. (b) Heat-induced evolution of normalized Raman spectra for $190 \mathrm{~nm}$ c-Si NP of the nanocavity and (c) BSA molecules inside. (d) Schematic of cancer cell uptake of polymer capsules loaded with anti-tumour drug with subsequent thermally triggered release via heating of $\alpha-\mathrm{Fe}_{2} \mathrm{O}_{3}$ NPs embedded into capsules walls with real-time temperature control by Raman scattering. (e) Confocal laser scanning microscopy images of the polymer capsules with $\alpha-\mathrm{Fe}_{2} \mathrm{O}_{3}$ nanoparticles in living cells. Inset shows SEM image of the capsule with $\alpha-\mathrm{Fe}_{2} \mathrm{O}_{3}$ nanoparticles. Cancer cells viability after incubation with polymer capsules modified with $\alpha-\mathrm{Fe}_{2} \mathrm{O}_{3}$ NPs and loaded with antitumor drug VCR (vincristine) for $24 \mathrm{~h}$ before (f) and after (g) irradiation with NIR laser. 
(a)
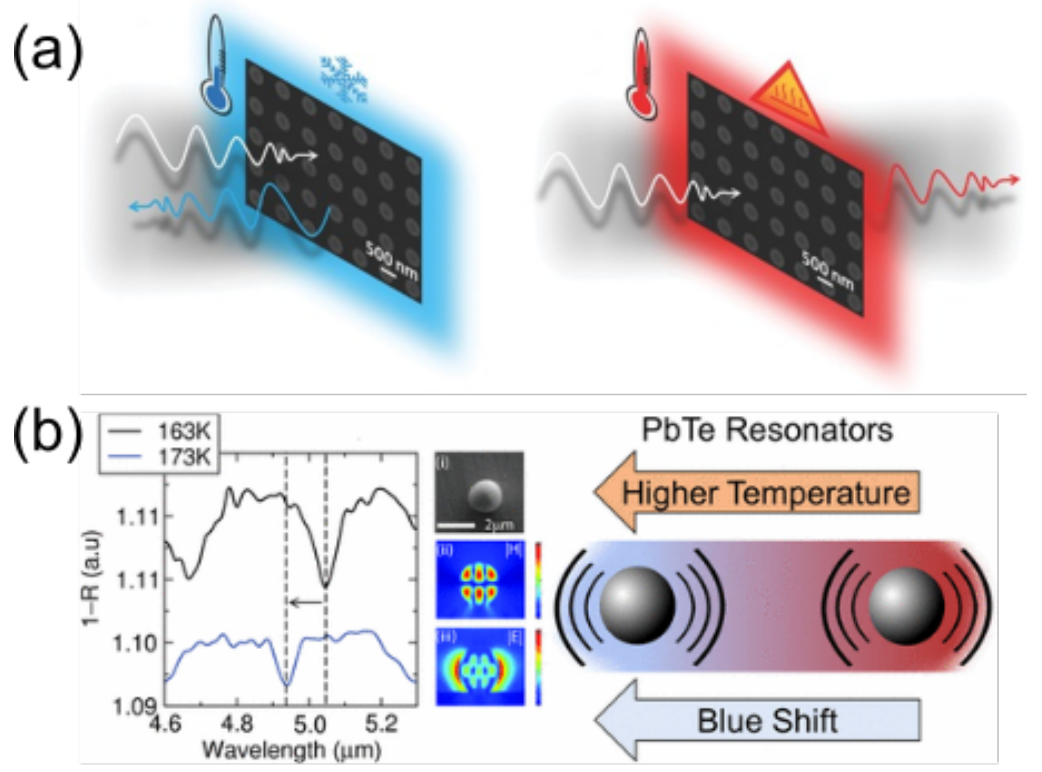

(c)
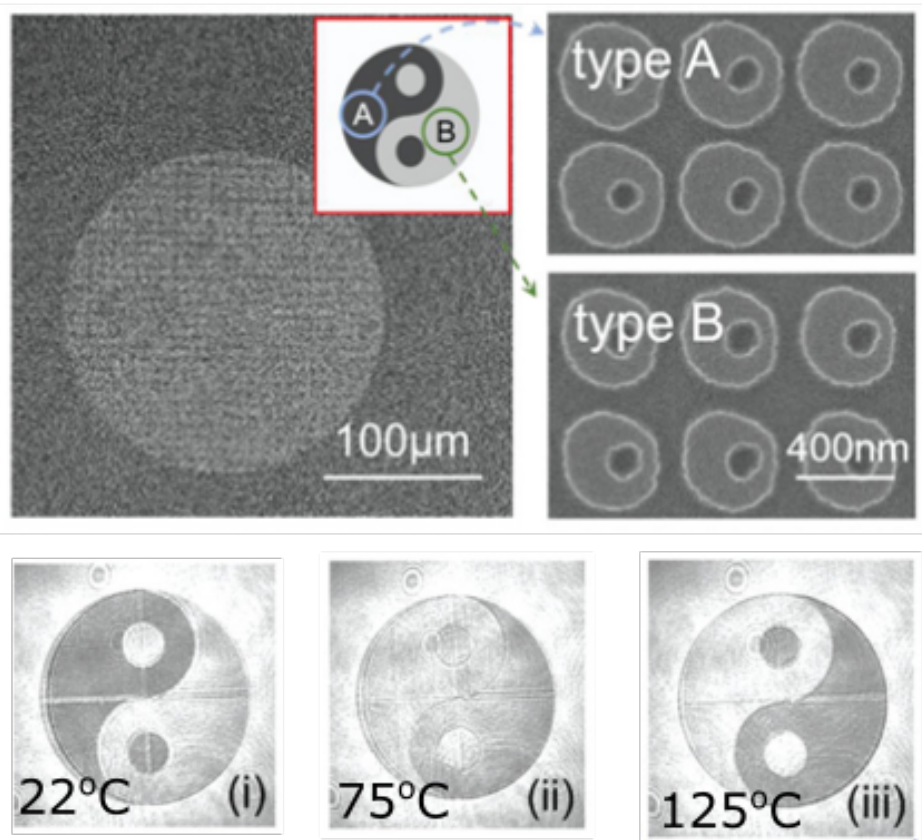

Figure 16: Thermal tuning of metaphotonic devices. (a) Illustration of the temperature effect on the far-field optical properties of metasurfaces by employing the actual scanning electron microscope (SEM) image. By cooling or heating the metasurface sample, one can operate in either the reflection or transmission regime, respectively [204]. (b) Ultrawide spectral tuning of resonances via heating of semiconductor meta-atoms [205]. (c) The SEM images of the fabricated Yin-Yang pattern with slightly different geometries of the two parts. The optical images of the metasurfaces obtained at $784 \mathrm{~nm}$ at different temperatures [206]. 
Fermi level, gives the most significant contribution in thermo-optical effect in semiconductor materials. Another contribution is related to the increased scattering rate of electrons in solids due to the enhanced phonon scattering, however this mechanism in more important in metals where the electron concentration stays almost constant.

The thermally induced refractive index change has real and imaginary parts being linearly dependent on temperature given by the following expression:

$$
\Delta n=\Gamma\left(T-T_{0}\right), \quad \Gamma=\left.\frac{d n}{d T}\right|_{T_{0}}
$$

where $\Gamma$ is the complex thermo-optical coefficient. Silicon has relatively high $\Gamma$, the real part of the $\Gamma$ is equal to $4.5 \cdot 10^{-4} \mathrm{~K}^{-1}$ and the imaginary part is equal to $0.1 \cdot 10^{-4} \mathrm{~K}^{-1}$ [208, whereas GaAs has four times smaller corresponding values. The values of the thermo-optical coefficients are summarized in Table 1. One can notice that the most of the semiconductor materials have positive real part of $\Gamma$. Thermal expansion leads to longer interatomic distances and, thus, weaker interaction between the electronic states and consequent decrease in the band gap. The well-known general law describing the temperature dependence of the band gap is as follows:

$$
E_{g}(T)=E_{g}(0)-\frac{\xi_{1} T^{2}}{T+\xi_{2}},
$$

where $\xi_{1}$ and $\xi_{2}$ are material dependent constants. The simple reasons based on thermal expansion provide that for the majority of materials $\xi_{1}>0$, which results in positive thermo-optical coefficient $\Gamma^{\prime}>0$. However, there is a number of materials where the band gap temperature dependence has anomalous form and, for instance, $\xi_{1}<0$ resulting in $\Gamma^{\prime}<0$. One can see that $\mathrm{PbTe}$ is one of that type of materials with negative of thermo-optical constant being several times higher in absolute values [205, 209], comparing to others materials. Recently, high negative thermo-optical constant of lead halide perovskites was also reported [178].

From the point of resonant nanoscale heating structure, the temperature change of the refractive index leads to thermal drift of the resonant wavelength:

$$
\frac{\Delta \lambda_{0}}{\lambda_{0}} \sim\left(\frac{1}{n} \frac{d n}{d T}+\frac{1}{D} \frac{d D}{d T}\right) \Delta T .
$$


Here, the first term corresponds to thermo-optical coefficient, while the second one is related to thermal expansion coefficient and related change in geometrical size of the nanostructure. From Table 1 one can see that normally the thermal expansion coefficient is smaller than the thermo-optical constant, but the contributions to the relative wavelength shift may be comparable. The recent findings show that among other materials perovskite structures may posses quite high value of thermal expansion coefficient $250 \cdot 10^{-6}$ $\mathrm{K}^{-1}[179]$.

Remarkably, typical raising time of the thermal nonlinearity is governed by electron-phonon scattering timescale laying in the interval of 1-10 ps [210]. The relaxation time in this case is slower than for optically induced nonlinearities caused by Kerr effects [211] or free carriers [212, 213] generation being usually about 1-100 ns [129] strongly depending on the thermal conductivity of surrounding medium.

In the case of a single resonant nanoparticle, it was shown theoretically that the photo-induced thermo-optical effect can lead to a self-consistent nonlinear heating [214].

Thermo-refractive optical nonlinearity was successfully utilized in various applications related to dramatic reversible changes of the optical properties of nanophotonic designs during temperature modulation.

In work [205], optical properties of microspheres from $\mathrm{PbTe}\left(\Gamma \approx-15 \cdot 10^{-4} \mathrm{~K}^{-1}\right)$ materials with Mie resonances were tuned in infrared range by varying temperature in range of $80-573 \mathrm{~K} 16 \mathrm{~b}$. This approach allowed to reversibly reconfigure all-dielectric nanoantennas resonances over their full-width, providing strong modulation of scattered/transmitted optical signal. In particular, it was demonstrated that high-quality factor Mie resonances can be tuned by several linewidths with temperature modulations as small as $\Delta \mathrm{T} \sim 10 \mathrm{~K}$.

Next step was done for all-dielectric metasurfaces, where the temperaturedependent change of the refractive index of silicon was employed to tune light transmission (see scheme in Fig. 16a) in a spectral window of $75 \mathrm{~nm}$ around the telecom wavelength. 204 The heating process resulted in a significant changes in the forward to backward light power propagation ratio from around 1 to more than 50 times. This is one of the highest reported value for tuning directionality by means of a reversible technique.

Basing on this approach, reversible image tuning was demonstrated with temperature tunable dielectric metasurfaces. [206] A metasurface with the encoded transmission Yin-Yang pattern (see Fig. 16k) was heated up by just $100 \mathrm{~K}$. Thermo-refractive nonlinear optical modulation of silicon properties 
was strong enough to change spectrally sharp Fano resonances of two sets of nanoresonators composed of nonconcentric silicon disks with holes (see SEM images in Fig. 16c). The authors achieved full control of the contrast of the Yin-Yang image in the reversible manner. Such thermally sensitive designs might be prospective for power limiters [215].

Stronger laser-induced heating of silicon allowed more than $+400 \%$ to $-90 \%$ nonlinear deviation of scattering from a single Mie-resonant silicon nanoparticle under continuous-wave (CW) illumination via enhanced thermorefractive nonlinearity. [129] The nanostructure can exhibit a strong nonlinear response (saturation of scattering). With a Gaussian focus, the nonlinear response should start from the center of point-source function, and thus, by extracting the nonlinear part, the resulting point-source function becomes smaller than its linear counterpart. It helped to beat diffraction limit by 2.3-times enhancement of optical imaging resolution enhancement as demonstrated in Fig. 17a.

In work [216], at the anapole wavelength in Si nanodisk the boosted nearfield energy directly contributes to the absorption, leading to a substantial temperature rise within the nanoparticle. In the temperature range from RT to $950{ }^{\circ} \mathrm{C}$, the change of the refractive index in real part $\Delta n$ is extrapolated to be 0.5 at a moderate laser intensity of $1.25 \times 10^{6} \mathrm{~W} / \mathrm{cm}^{2}$. This equivalently gives the effective nonlinear refractive index as $n_{2}(532 \mathrm{~nm})=\Delta n / \mathrm{I}=0.4$ $\mathrm{cm}^{2} / \mathrm{MW}$. Compared with the measured temperature rise in bulk Si, optical anapole significantly enhanced photothermal nonlinearity by three orders of magnitude.

Finally, Raman thermometry can be applied for mapping the non-uniformly heated Si pillars with the sub-diffraction lateral resolution. [133] As shown in Figure 17k, Raman maps obtained at incident beam intensities of 4 and $42 \mathrm{~mW} / \mu \mathrm{m}^{2}$ for Si pillar dimer. At $\mathrm{I}=4 \mathrm{~mW} / \mu \mathrm{m}^{2}$, which corresponds to almost the "cold" structure, no specific features can be resolved in the acquired Raman map. On the other hand, at $\mathrm{I}=42 \mathrm{~mW} / \mu \mathrm{m}^{2}$, the Raman signal distribution at $520 \mathrm{~cm}^{-1}$ shows a decrease in the intensity in the central area of each Si pillar, which is attributed to the pronounced temperature dependent spectral shift of the corresponding c-Si band in this particular area upon its more efficient heating. Remarcably, the Raman imaging technique also allows for simultaneous monitoring of the material properties for complex compositions (e.g. like SiGe [217]).

All these works on thermal-nonlinearity enhanced optical imaging demonstrated comparable resolution improvement down to $\lambda / 10$, extending the ap- 


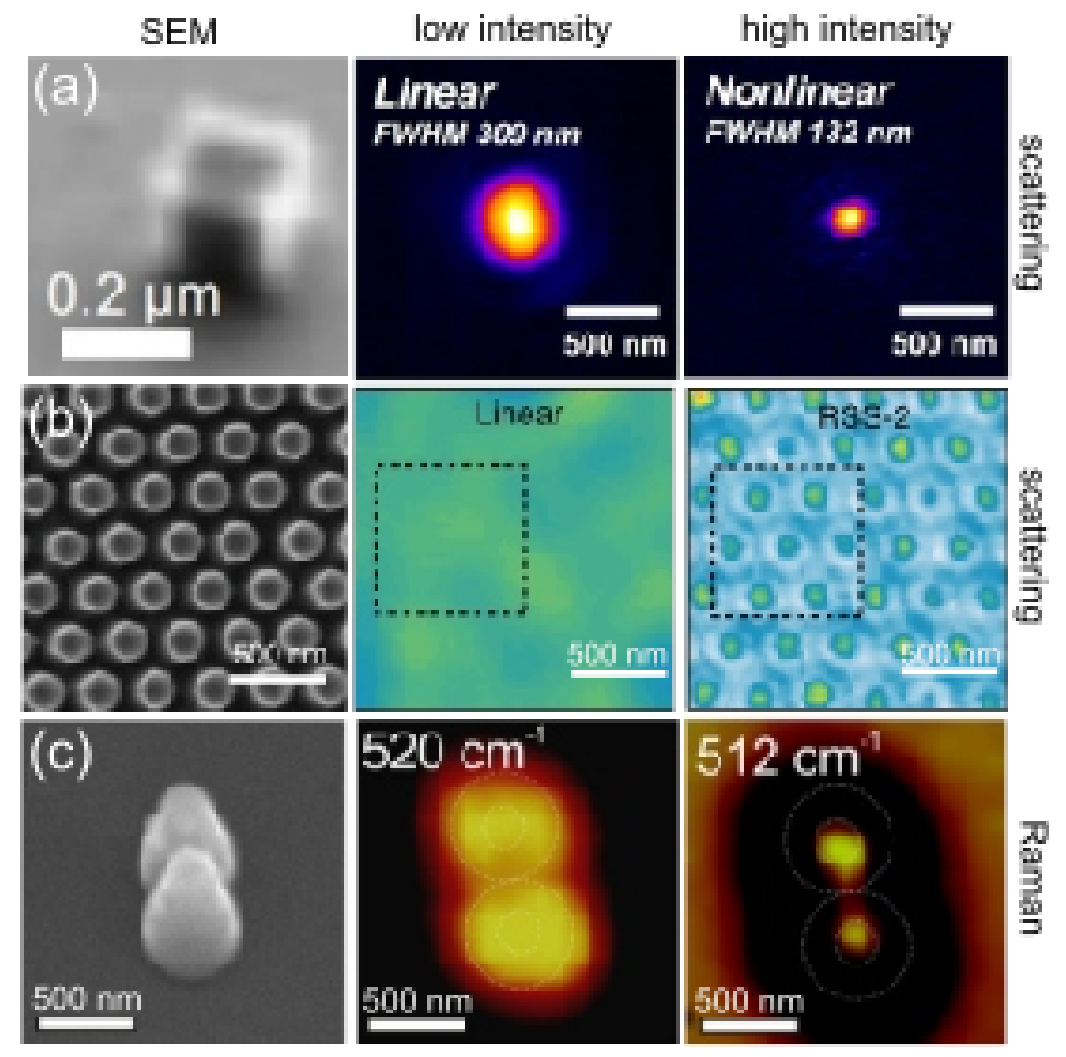

Figure 17: Superresolution imaging of Si nanoparticles with thermooptical nonlinearity. (a) SEM image of a Si nanocube on a quartz substrate, and optical images of scattered signal from a nanoscale cube at low (linear regime) and high (nonlinear regime, $\sim 6 \mathrm{~mW} / \mu \mathrm{m}^{2}$ at wavelength $592 \mathrm{~nm}$ ) intensities of incident CW laser. [129] (b) Confocal optical images of nonlinear scattering from periodic Si nanodisk arrays with anapole states evolve with increasing excitation intensities and correlated SEM image. The optical super-resolution image is a differential between two images, where the nonlinear regime corresponds to $15 \mathrm{~mW} / \mu \mathrm{m}^{2}$ at wavelength $532 \mathrm{~nm}$. [216] (c) Side-view SEM images of the Si pillar dimer, as well as corresponding Raman scattering maps at $520 \mathrm{~cm}^{-1}$ and $512 \mathrm{~cm}^{-1}$ measured at the incident intensities of 4 and $42 \mathrm{~mW} / \mu \mathrm{m}^{2}$ at wavelength $532 \mathrm{~nm}$, respectively. [133] 
plication of super-resolution microscopy not only to label-free silicon nanostructure observations but also further into biomedical applications with silicon nanoparticles.

\subsection{Thermally-induced phase transitions}

Further increase of the temperature range leads to inherent phase transitions in the materials associated with a change of material parameters, when the local temperature of a sample exceeds a threshold value. The most of such phase transitions are volatile so that a material returns to its initial phase upon cooling. One of the most common example is melting/crystallization process.

Another class of phase transitions, namely transitions between crystalline and amorphous phases, paves the way for designing a non-volatile photonic nanostructures made of materials with a glass-transition effect. Amorphous phase generally has higher configuration entropy than the lowest free-energy state in crystalline phase. At the same time, below the melting temperature $T_{m}$ viscosity exhibiting strong increase with the decreasing of the temperature suppresses atomic diffusion at the glass-transition temperature $T_{g}$. Thus, a rapid quenching of melted material (the cooling rate higher than crystalline rate) prohibits atoms to form an ordered lattice, resulting in formation of a quasi-stable amorphous phase. In the case of nanophotonic structures, a femtosecond optical pulse that delivers energy to material for heating it above $T_{m}$ succeeded by quenching the sample below the glass-transition temperature $T_{g}$ leads to freeze a disorder making the transition non-volatile. However, this process can be reversed. A train of pulses, each of them heats the antenna to the temperature above $T_{g}$ but below $T_{m}$, allows transforming the material back into a crystalline phase through a sequence of amorphous phases with decreasing disorder.

Non-volatile phase transitions were demonstrated for silicon nanospheres fabricated by a laser ablation method, i.e. a femtosecond laser printing [218, 81. Amorphous (a-Si) to crystalline (c-Si) transitions in silicon leads to a decrease of dielectric permittivity in the spectral range from $500 \mathrm{~nm}$ to 900 $\mathrm{nm}$. The physical origin of this behavior is the following. In c-Si, electron transitions in the interval from 365 to $1130 \mathrm{~nm}$ are indirect, that is they take place between states of different wave vector with the simultaneous absorption or emission of a phonon. At the same time the lack of wave vector conservation in a-Si makes these transitions quasi-allowed resulting in 


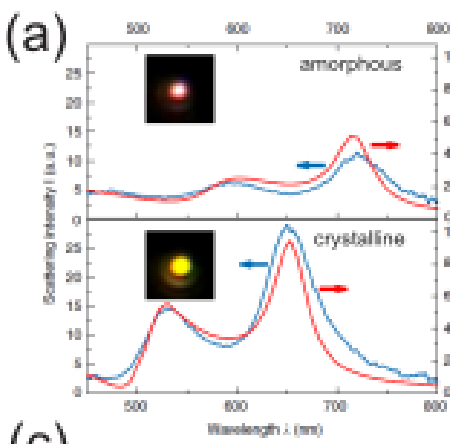

(b)

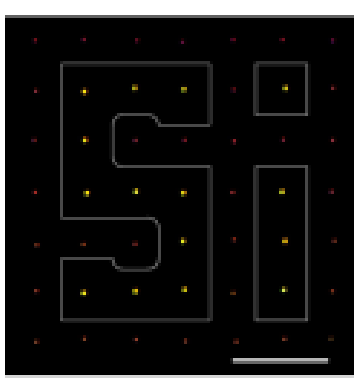

(c)
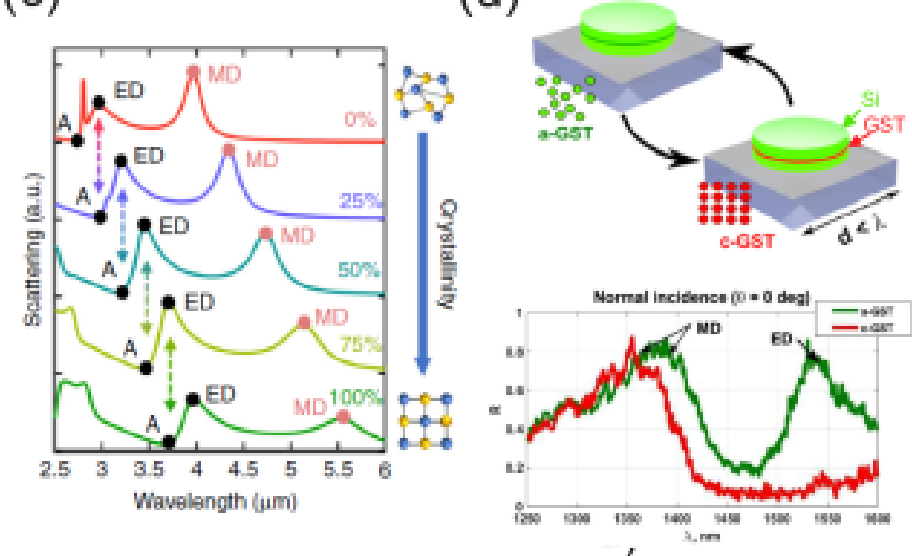

Figure 18: Nanoparticles made of phase-changing materials. (a) Experimental (blue curves) and theoretical (red curves) scattering spectra, calculated using Mie theory, of spherical Si nanoparticles. (b) Dark-field microscopic image of the laser-printed Si nanoparticles. The nanoparticles within the white lines are crystallized by additional laser pulse irradiation, producing a visible colour change (scale bar, $10 \mu \mathrm{m}$ ) [218]. (c) Numerically solved conditions different bright and dark states under different crystallinities of a GST nanodisk [219]. (d) hybrid Si/GST cylinders effectively behave as Sionly when the GST is amorphous, and the resonant modes supported by the array (thus its optical response) can be modified on demand by switching the GST layer between its amorphous and crystalline states and experimentally obtained reflectance spectra for the as-fabricated device with the GST layer in both amorphous and crystalline states [220]. 
stronger optical response. Also there are differences in the density of valence states, which also account for the longer wavelength shift in the maximum of the imaginary part of $\varepsilon$ [221]. This moderate change of the permittivity (from $\varepsilon_{\mathrm{a}-\mathrm{Si}}=16.5$ to $\varepsilon_{\mathrm{c}-\mathrm{Si}}=14$ at wavelength $\lambda \approx 700 \mathrm{~nm}$ ) can shift a position of the Mie resonance up to $70 \mathrm{~nm}$ [218]. By using the femtosecond laser printing, Zywietz et al. [218] fabricated a square lattice (lattice spacing 5 $\mu \mathrm{m})$ of similar a-Si nanoparticles that have the magnetic dipole Mie resonance at $\lambda \approx 720 \mathrm{~nm}$. Next, the laser-induced crystallization in the array of these a-Si nanoparticles allowed for selective change of the properties of single nanoparticles. After the crystallization, the Mie resonance is shifted to $\lambda \approx$ $650 \mathrm{~nm}$ since the silicon permittivity is decreased due to the laser-induced phase transition (see Fig. 18a). This behavior was also employed to modify locally optical properties of individual a-Si nanoparticles to create a picture "Si" as shown in Fig. 18b.

Further, the approach of local thermally-induced phase switching was applied to record various complicated colorful patterns with amorphous $\mathrm{Si}$ nanoparticles prepared by nanolithography. 222] This method is quite useful, because it allows for creation of c-Si nanoparticles on an arbitrary substrate (i.e. like glass) without employing silicon-on-insulator or silicon-on-sapphire technologies, as well as any transfer techniques. Additionally, this technique allowed for local laser annealing of amorphous silicon nanoparticles with in situ control of temperature and crystalline state via generated Raman signal analysis [223].

By using another important compound based on germanium (Ge) - antimony ( $\mathrm{Sb}$ ) - tellurium (Te) alloys, and often referred to as GST, one can also achieve non-volatile transitions. Recently, such GST alloys catch a lot of attention in photonics since their dielectric properties demonstrate a very strong modulation. In contrast to the moderate change in optical and electrical properties of amorphous and crystalline phases of $s p^{3}$-bonded semiconductors such as silicon, GST alloys possess unsaturated covalent bonds leading for a resonant bonding to exist in crystalline phase [224]. The resulting ground state can be explained as a superposition of symmetricallyequivalent states with saturated-bond configurations. Thus, electrons are effectively delocalized resulting in a high dielectric permittivity values. On the other hand, the resonant bonding requires the long-range ordering and in amorphous phase this ordering is not possible causing a strong contrast in optical properties of a-GST and c-GST alloys [225. Due to low losses for $\lambda>1.5 \mu \mathrm{m}$ the GST-based nanophotonic designs employing optical reso- 
nances can operate in the near-infrared range.

Wang et al. 226] reported on a dipolar metasurface operating around a wavelength of $\lambda=2 \mu \mathrm{m}$, where absorption in GST is low enough to achieve resonances. They designed the metasurface comprising a two-dimensional array of rectangular crystalline inclusions in the amorphous GST film. Both transmission and reflection spectra show the resonant feature at $\lambda=2 \mu \mathrm{m}$ for light polarized along the inclusion and no dips or peaks for the orthogonal polarization. For wavelengths $\lambda>1.78 \mu \mathrm{m}$ the structure does not scatter light in the non-zero diffraction orders [227] demonstrating a true metamaterial nature [226].

In work [219], it was demonstrated that the structured phase-change alloy $\mathrm{Ge}_{2} \mathrm{Sb}_{2} \mathrm{Te}_{5}$ (225-GST) can support a diverse set of multipolar Mie resonances with active tunability. By harnessing the dramatic optical contrast of GST, broadband $(\Delta \lambda / \lambda \sim 15 \%)$ mode shifting between an electric dipole resonance and an anapole state (see Fig. 18c). Active control of higher-order anapoles and multimodal tuning were also investigated, which make the structured GST serve as a multi-spectral optical switch with high extinction contrasts $(>6 \mathrm{~dB})$.

In order to shift from IR to visible range, a new concept in all-dielectric optical metasurfaces introduced and experimentally validated based on a hybrid combination of high-index and low-loss dielectric building blocks with embedded subwavelength inclusions of chalcogenide phase-change materials. [220] By using this hybrid approach, the authors were able not only to provide on-demand dynamic control of light amplitude, but also to deliver a very high efficiency of operation over a very wide spectral range by a judicious material choice. The authors demonstrated the flexibility and universality of our approach by the design and development of hybrid metasurfaces for applications as switchable spectral filters in the near-infrared and dynamic color generation in the visible spectrum.

\subsection{Optical reshaping}

One of the most promising applications of dielectric nanoparticle reshaping is the data storage, color printing, and data storage, where the present memory limit due to the current density has to be surpassed for the substantial growth of data traffic and archive applications. Indeed, according to many studies, all-dielectric nanophotonic designs exhibit optical properties very sensitive to their shape. [230, 231, 232, 233] 

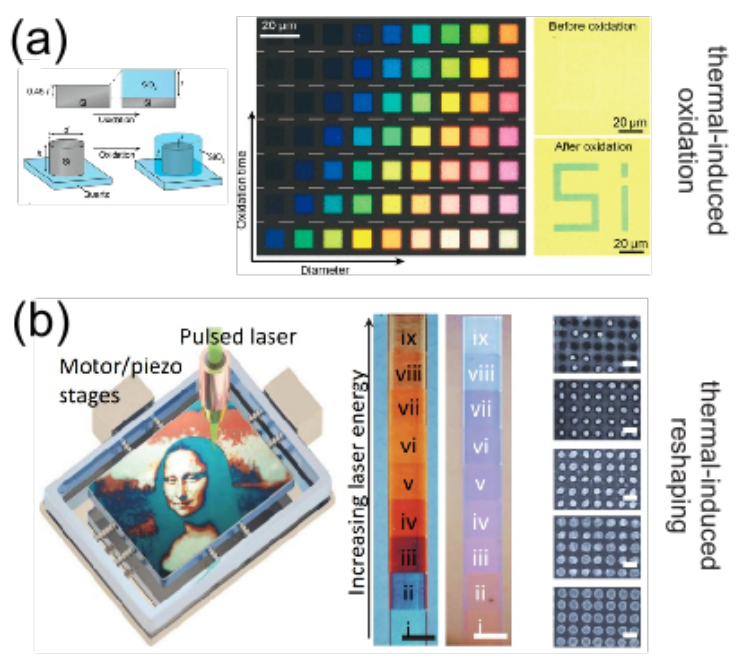

(c)
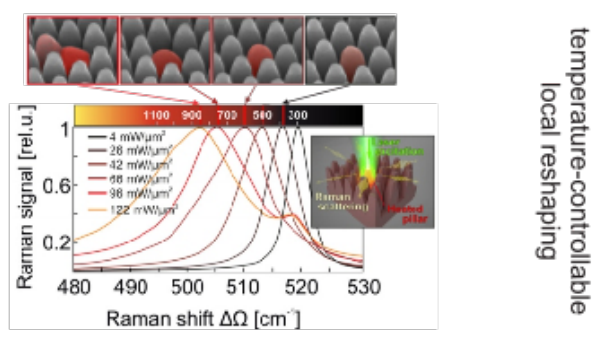

Figure 19: (a) Schematic of the calculation model for Si nanostructure oxidation. Reflection image of oxidized arrays through a $20 \times$ objective $(\mathrm{NA}=0.45)$ irradiated with linear polarized white light. The diameter was systematically changed from $90 \mathrm{~nm}$ to $250 \mathrm{~nm}$ in $20 \mathrm{~nm}$ increments. The vertical axes show total oxidation time and $\mathrm{SiO}_{2}$ layer thickness. Each individual color area is $10 \mu \mathrm{m} \times 10 \mu \mathrm{m}$. The black frame corresponds to unstructured regions. The structures shown in each row are the same, with the extent of oxidation increasing toward the top of the image. Scale bar, $10 \mu \mathrm{m}$. [228] (b) Schematic setup of resonant laser printing. Synchronous motion solution with the laser pulses is provided by computer-controlled motor or piezostages. Reflection and transmission micro-images of multicolored structures generated by gradually increasing laser powers. Microstructures (i to ix) are generated under gradually increasing laser power strengths from 0.2 to $1.8 \mathrm{~mJ}$ in steps of $0.2 \mathrm{~mJ}$, controlled using a liquid crystal attenuator. Scale bars, $0.5 \mathrm{~mm}$. Corresponding SEM images of the microstructures (i, iii, v, vii, and ix), showing the change of the morphology of the unit cell from disk to sphere and eventually a hole. Scale bars, $200 \mathrm{~nm}$. Gamut loop of laser-printed structural colors, which covers CMY colors. [229] (c) Temperature-feedback laser reshaping of Si resonators. SEM\$mages show evolution of the geometric shape of the $\mathrm{Si}$ nanoparticle upon heating with a CW laser at intensity increasing from 4 to $122 \mathrm{~mW} / \mu \mathrm{m}^{2}$. Bottom: Corresponding thermal-induced shift of the c-Si Raman band upon laser heating of the isolated Si resonator. The inset shows schematic of the laser heating and Raman signal generation processes. [133] 
At higher temperatures, some intermediate case can be realized, when irreversible phase transition (e.g. oxidation) causes change of nanoparticle shape and design. In work [228], lithographically fabricated Si nanoparticles were thermally oxidized in a furnace at $750{ }^{\circ} \mathrm{C}$ with introducing saturated steam and air at atmospheric pressure to form an oxidized layer on the nanostructure surface. At room temperature, the Si interior is protected by the formation of a native thin oxide film $\left(\mathrm{SiO}_{2}\right)$, even if exposed to air for a long time. 234 However, with a sufficient amount of steam and oxygen at high temperatures, molecules diffuse through the oxide film and react with $\mathrm{Si}$ at the $\mathrm{Si} / \mathrm{SiO}_{2}$ interface according to the reactions $\mathrm{Si}+\mathrm{O}_{2} \rightarrow \mathrm{SiO}_{2}$ and $\mathrm{Si}+2 \mathrm{H}_{2} \mathrm{O} \rightarrow \mathrm{SiO}_{2}+2 \mathrm{H}_{2}$, resulting in an approximately 2.2-fold volume expansion of the oxide film, as shown in schematic of Fig. 19a. The Si nanostructure arrays with controllable oxide layer exhibited distinct and vivid colors, which were strongly dependent on the oxidation time. Optical image in Figure 19 a shows wide gamut of the created Si metasurface colors, as well as the ability to the hidden pattern development after applying high temperature. Generally, compared to plasmonic analogs, color surfaces with high-index dielectrics, such as $\mathrm{Si}$ or Ge, have a lower reflectance, yielding a superior color contrast. [235, 236, 237, 238, 239, 240, 241]

In the case of absence of considerable chemical reactions during nanostructure heating, a dewetting mechanism related to the minimization of the total energy surfaces occurs [242, 243, 244]. The dewetting is a prospective tool for large-scale nanofabrication and thermally-induced reshaping of dielectric nanoparticles. [245, 246, 238, 247] The laser-matter interaction responsible for the reshaping via dewetting is also related to local melting [248, 249]. This concept was successfully realized for laser-postprocessing of a Ge metasurfaces with morphology-dependent resonances and color [229], as shown in Fig. 19b. Moreover, the authors used a polarization-sensitive color palette to create complex colorized images at resolutions beyond the diffraction limit. By elongating the disks into bars they showed that the asymmetric structures can support tunable color under polarized incident light in the visible spectrum. The patterns were printed with a resolution of 100000 DPI. 229] Similar concept was demonstrated with Si nanostructures. [250] Laser-induced dewetting was also applied for fabrication of metasurface spanning across $10^{2}-10^{4} \mu \mathrm{m}^{2}$ and, subsequently, consisting of thousands of polycrystalline Si nanostructures distributed in an ordered rectangular lattice. 251.

Remarkably, that according to Section 4 on nanothermometry, the laser- 
induced nanoparticles reshaping can be carried out with precise temperature control by doing nanothermometry via measuring in situ Raman signal during the process. Figure 19 c shows the correlation between shape of Si nanoparticle and its Raman spectrum exhibiting temperature-driven shift allowing to determine actual temperature during the reshaping. [133] Additional spatial Raman mapping of the heated nanoparticle with subdiffractional resolution revealed stronger heating of their top parts relatively the bottom ones.

\section{Conclusion and outlook}

We have demonstrated how all-dielectric nanophotonics can be employed as a promising low-loss platform for light-matter manipulation at the nanoscale, more specifically as a powerful tool for subwavelength optical heating. The ability to tune precisely optical losses in dielectrics in a broad spectral range allows to achieve optimal conditions for light absorption in nanostructures employing the critical coupling concept when radiative and nonradiative losses become equal. Here, we have reviewed different advanced techniques recently emerged in nanothermometry dealing with all-dielectric nanoscale structures, which make such nanostructures perfect candidates for the development of 'all-in-one' platforms for simultaneous optical heating, thermom-

etry, and additional photo-induced manipulation of various objects at the nanoscale.

We anticipate a rapid progress of the field of all-dielectric thermonanophotonics and its applications in photonics, optoelectronics, chemistry, and biomedicine. Below, we mention just a few example of the expected developments.

First, further progress should be demonstrated in nanothermometry, where novel designs and materials for highly sensitive temperature measurements at the nanoscale (such as resonant nanodiamonds with NV-centers [252, 253] empowered by Mie resonances) can play an important role. For example, the optimization of optical tweezers for operating with various nanoscale objects would enable simultaneous temperature control [120, 135] providing a novel platform for advanced changing and probing of local temperature via all-optical manipulation with nanothermometry.

In nonlinear optics, thermo-modulated nonlinear nanophotonic designs for harmonics generation and multiphoton photoluminescence can be developed based on thermally-driven variation of the refractive index or light 
emission quantum efficiency. It can open new directions such as thermally tunable nonlinear flat optics for IR imaging and LIDAR applications.

In optoelectronics, smart nanostructuring of semiconductors can lead to a breakthrough in the optimization of thermo-electrical effects, [254] where the optimal absorption and temperature distribution at nano-/microscales are highly desirable. Moreover, thermal management is crucial for optimization of nanolasers and nano-LEDs, where overheating is a parasitic effect reducing light-emitting properties.

Strong subwavelength near-field localization and fast optical heating are highly desirable for heat-assisted data storage (e.g. magnetic recording [255]). In this regard, advanced all-dielectric nanostructures may be useful for the precise optical heating with high spatial and temperature resolution, and they may allow for near-threshold operation of a recording device making the storage as dense as possible.

For applications dealing with functional liquids, we mention that a precise control on variation of local temperature in dielectric nanoparticles can be beneficial for temperature-induced liquid flows. Besides that, the generation of temperature gradients by nanoparticles along solid/liquid interfaces can lead to thermo-osmotic flows with the speeds exceeding $100 \mu \mathrm{m} \mathrm{s}^{-1}$, also being strongly confined to the interfacial region that is important for thermophoresis. Stronger heating is useful for photothermally-induced microbubble formation around nanoparticles, where a bubble can nucleate at the interface above a certain power. It can also be used for various biomedical applications such as cancer therapy, photo-acoustic imaging, or optoporation.

Chemistry is also the research field that can benefit from the use of various dielectric nanoparticles. Optically resonant dielectric nanoparticles allowing for in situ nanothermometry might find useful applications in photothermallyassisted chemical vapour deposition, photothermally-assisted catalysis in the gas phase, as well as photothermally-assisted enhanced reactivity in solutions.

Finally, various multidisciplinary directions can emerge from the development of thermonanophotonics employing the approaches from radiophysics, where heating due to electromagnetic waves was very well known for many decades, and now it inspires nanophotonics and multidisciplinary communities to find new effects and completely unexpected applications. For example, we mention the recent studies of the formation of plasma due to electromagnetic hot-spots arising from the cooperative interaction of Mie resonances in the individual spheres, suggesting a method to experimentally model subwavelength field patterns using thermal imaging in macroscopic dielectric 
systems [256]).

\title{
Acknowledgements
}

This work was supported by the Russian Science Foundation (project 21-7530020) and the Australian Research Council (grant DP200101168).

\section{Appendix. Symbols and Acronyms}

\author{
NP Nanoparticle \\ DF Dark-field scattering \\ CW Continuous-wave laser \\ ED, EQ, EO Electric dipole, quadrupole and octopole modes \\ MD, MQ, MO Magnetic dipole, quadrupole and octopole modes \\ SRS Stimulated Raman scattering \\ SEM Scanning electron microscopy \\ TEM Transmission electron microscopy \\ AFM Atomic force microscopy \\ SERS Surface-enhanced Raman scattering \\ J Electric current \\ $C_{e}, C_{i} \quad$ Specific heat capacity of electron and lattice subsystems \\ $T_{e}, T_{i}$ Temperature of electron and lattice subsystems \\ $\gamma_{e i}$ Coupling factor of electron-phonon interaction \\ $N_{e}, N_{t h}$ Conduction band electron density and critical carrier \\ density \\ $G_{e} \quad$ Electron generation rate \\ $R_{e}$ Electron relaxation rate \\ $\mu_{e} \quad$ Electron mobility \\ $\tau_{0}, \tau_{\gamma}$ Hot carrier relaxation time and total electron-phonon \\ relaxation time \\ $K_{c} \quad$ Cooling rate \\ $\rho, c_{p}, \kappa$ Material density, heat capacity at constant pressure and \\ thermal conductivity
}


$\alpha, \chi \quad$ Absorption coefficient and thermal diffusivity $\left(\kappa / \rho c_{p}\right)$

$Q$ and total dissipated power

$R$ Reflection coefficient

$I, I_{0}, \mathfrak{P}$ Intensity distribution inside the medium, incident light intensity and $\mathfrak{P}=a^{2} I_{0}$ is total power of Gaussian beam with $a$ radius and $I_{0}$ intensity

$T, T_{0}, T^{\prime}$ Temperature at specific point of the material, initial temperature and the temperature increase under laser irradiation $\left(T-T_{0}\right)$

$C_{s c a}, C_{e x t}, C_{a b s}$ Scattering, extinction and absorption cross-sections of light

$a_{l}, b_{l} \quad$ Electric and magnetic scattering Mie-coefficients of different order

$\tau_{p}$ Pulse duration

$\lambda$ Wavelength of light in free space

$\varepsilon, \varepsilon^{\prime}, \varepsilon^{\prime \prime}$ Total, real part and imaginary part of dielectric permittivity

$\gamma_{\text {rad }}, \gamma_{\text {Ohmic }}$ Radiative and nonradiative (Ohmic) optical losses of the system

$\mathrm{n}$ Refractive index 


\section{References}

[1] Jeremy J Baumberg, Javier Aizpurua, Maiken H Mikkelsen, and David R Smith. Extreme nanophotonics from ultrathin metallic gaps. Nature Materials, 18(7):668-678, 2019.

[2] Vincenzo Giannini, Antonio I Fernández-Domínguez, Susannah C Heck, and Stefan A Maier. Plasmonic nanoantennas: fundamentals and their use in controlling the radiative properties of nanoemitters. Chemical Reviews, 111(6):3888-3912, 2011.

[3] Jon A Schuller, Edward S Barnard, Wenshan Cai, Young Chul Jun, Justin S White, and Mark L Brongersma. Plasmonics for extreme light concentration and manipulation. Nature Mater., 9(3):193-204, 2010.

[4] Martti Kauranen and Anatoly V Zayats. Nonlinear plasmonics. Nature Photonics, 6(11):737-748, 2012.

[5] Harry A Atwater and Albert Polman. Plasmonics for improved photovoltaic devices. Nature Mater., 9(3):205-213, 2010.

[6] Guillaume Baffou, Frank Cichos, and Romain Quidant. Applications and challenges of thermoplasmonics. Nature Materials, pages 1-13, 2020 .

[7] Xiaohua Huang, Prashant K Jain, Ivan H El-Sayed, and Mostafa A El-Sayed. Plasmonic photothermal therapy (pptt) using gold nanoparticles. Lasers in medical science, 23(3):217-228, 2008.

[8] Umar Aslam, Vishal Govind Rao, Steven Chavez, and Suljo Linic. Catalytic conversion of solar to chemical energy on plasmonic metal nanostructures. Nature Catalysis, 1(9):656-665, 2018.

[9] Guillermo González-Rubio, Pablo Díaz-Núñez, Antonio Rivera, Alejandro Prada, Gloria Tardajos, Jesús González-Izquierdo, Luis Bañares, Pablo Llombart, Luis G Macdowell, Mauricio Alcolea Palafox, et al. Femtosecond laser reshaping yields gold nanorods with ultranarrow surface plasmon resonances. Science, 358(6363):640-644, 2017.

[10] Guillaume Baffou, Romain Quidant, and F Javier García de Abajo. Nanoscale control of optical heating in complex plasmonic systems. ACS Nano, 4(2):709-716, 2010. 
[11] Alexander O Govorov and Hugh H Richardson. Generating heat with metal nanoparticles. Nano Today, 2(1):30-38, 2007.

[12] Guillaume Baffou and Hervé Rigneault. Femtosecond-pulsed optical heating of gold nanoparticles. Physical Review B, 84(3):035415, 2011.

[13] Michael I Tribelsky, Andrey E Miroshnichenko, Yuri S Kivshar, Boris S Luk'yanchuk, and Alexei R Khokhlov. Laser pulse heating of spherical metal particles. Physical Review X, 1(2):021024, 2011.

[14] Guillaume Baffou and Romain Quidant. Nanoplasmonics for chemistry. Chem. Soc. Rev., 43:3898-3907, 2014.

[15] Satoshi Ishii, Ramu Pasupathi Sugavaneshwar, and Tadaaki Nagao. Titanium nitride nanoparticles as plasmonic solar heat transducers. The Journal of Physical Chemistry C, 120(4):2343-2348, 2016.

[16] George Ni, Nenad Miljkovic, Hadi Ghasemi, Xiaopeng Huang, Svetlana V Boriskina, Cheng-Te Lin, Jianjian Wang, Yanfei Xu, Md Mahfuzur Rahman, TieJun Zhang, et al. Volumetric solar heating of nanofluids for direct vapor generation. Nano Energy, 17:290-301, 2015.

[17] Peng Tao, George Ni, Chengyi Song, Wen Shang, Jianbo Wu, Jia Zhu, Gang Chen, and Tao Deng. Solar-driven interfacial evaporation. Nature energy, 3(12):1031-1041, 2018.

[18] Zhiguang Zhou, Enas Sakr, Yubo Sun, and Peter Bermel. Solar thermophotovoltaics: reshaping the solar spectrum. Nanophotonics, 5(1):121, 2016.

[19] Zunaid Omair, Gregg Scranton, Luis M Pazos-Outón, T Patrick Xiao, Myles A Steiner, Vidya Ganapati, Per F Peterson, John Holzrichter, Harry Atwater, and Eli Yablonovitch. Ultraefficient thermophotovoltaic power conversion by band-edge spectral filtering. Proceedings of the National Academy of Sciences, 116(31):15356-15361, 2019.

[20] Peter Zijlstra, James WM Chon, and Min Gu. Five-dimensional optical recording mediated by surface plasmons in gold nanorods. Nature, 459(7245):410-413, 2009. 
[21] Anders Kristensen, Joel KW Yang, Sergey I Bozhevolnyi, Stephan Link, Peter Nordlander, Naomi J Halas, and N Asger Mortensen. Plasmonic colour generation. Nature Reviews Materials, 2(1):1-14, 2016.

[22] Jinxing Chen, Zuyang Ye, Fan Yang, and Yadong Yin. Plasmonic nanostructures for photothermal conversion. Small Science, 1:2000055, 2021.

[23] Miao Li, Theobald Lohmuller, and Jochen Feldmann. Optical injection of gold nanoparticles into living cells. Nano letters, 15(1):770-775, 2015 .

[24] Costas M Pitsillides, Edwin K Joe, Xunbin Wei, R Rox Anderson, and Charles P Lin. Selective cell targeting with light-absorbing microparticles and nanoparticles. Biophysical journal, 84(6):4023-4032, 2003.

[25] Leon R Hirsch, R Jason Stafford, JA Bankson, Scott R Sershen, B Rivera, RE Price, John D Hazle, Naomi J Halas, and Jennifer L West. Nanoshell-mediated near-infrared thermal therapy of tumors under magnetic resonance guidance. Proceedings of the National Academy of Sciences, 100(23):13549-13554, 2003.

[26] Jaber Beik, Maziar Khateri, Zohreh Khosravi, S Kamran Kamrava, Siavash Kooranifar, Habib Ghaznavi, and Ali Shakeri-Zadeh. Gold nanoparticles in combinatorial cancer therapy strategies. Coordination Chemistry Reviews, 387:299-324, 2019.

[27] Guangyu Qiu, Zhibo Gai, Yile Tao, Jean Schmitt, Gerd A KullakUblick, and Jing Wang. Dual-functional plasmonic photothermal biosensors for highly accurate severe acute respiratory syndrome coronavirus 2 detection. ACS nano, 14(5):5268-5277, 2020.

[28] Maria Pihl, Ellen Bruzell, and Martin Andersson. Bacterial biofilm elimination using gold nanorod localised surface plasmon resonance generated heat. Materials Science and Engineering: C, 80:54-58, 2017.

[29] Dilip Y Paithankar, Fernanda H Sakamoto, William A Farinelli, Garuna Kositratna, Richard D Blomgren, Todd J Meyer, Linda J Faupel, Arielle NB Kauvar, Jenifer R Lloyd, Wang L Cheung, et al. Acne treatment based on selective photothermolysis of sebaceous follicles 
with topically delivered light-absorbing gold microparticles. Journal of Investigative Dermatology, 135(7):1727-1734, 2015.

[30] Todd James Harris and Alice Ann Chen Kim. Hair removal with coated metal nanoparticles, August 23 2016. US Patent 9,421,259.

[31] Ariel M Wilson, Javier Mazzaferri, Éric Bergeron, Sergiy Patskovsky, Paule Marcoux-Valiquette, Santiago Costantino, Przemyslaw Sapieha, and Michel Meunier. In vivo laser-mediated retinal ganglion cell optoporation using kv1. 1 conjugated gold nanoparticles. Nano letters, 18(11):6981-6988, 2018.

[32] Mengistie L Debasu, Duarte Ananias, Isabel Pastoriza-Santos, Luis M Liz-Marzán, J Rocha, and Luís D Carlos. All-in-one optical heaterthermometer nanoplatform operative from 300 to $2000 \mathrm{k}$ based on er3+ emission and blackbody radiation. Advanced Materials, 25(35):48684874, 2013.

[33] Guillaume Baffou, Pascal Berto, Esteban Bermúdez Ureña, Romain Quidant, Serge Monneret, Julien Polleux, and Hervé Rigneault. Photoinduced heating of nanoparticle arrays. Acs Nano, 7(8):6478-6488, 2013.

[34] Aquiles Carattino, Martín Caldarola, and Michel Orrit. Gold nanoparticles as absolute nanothermometers. Nano Letters, 18(2):874-880, 2018.

[35] Arseniy I Kuznetsov, Andrey E Miroshnichenko, Mark L Brongersma, Yuri S Kivshar, and Boris Luk'yanchuk. Optically resonant dielectric nanostructures. Science, 354(6314):aag2472, 2016.

[36] Isabelle Staude and Jörg Schilling. Metamaterial-inspired silicon nanophotonics. Nature Photonics, 11(5):274, 2017.

[37] Isabelle Staude, Vyacheslav V Khardikov, Nche T Fofang, Sheng Liu, Manuel Decker, Dragomir N Neshev, Ting Shan Luk, Igal Brener, and Yuri S Kivshar. Shaping photoluminescence spectra with magnetoelectric resonances in all-dielectric nanoparticles. ACS Photonics, 2(2):172$177,2015$. 
[38] EY Tiguntseva, George P Zograf, Filipp E Komissarenko, Dmitry A Zuev, Anvar A Zakhidov, Sergey V Makarov, and Yuri S Kivshar. Light-emitting halide perovskite nanoantennas. Nano Letters, 18(2):1185-1190, 2018.

[39] Ekaterina Y Tiguntseva, Denis G Baranov, Anatoly P Pushkarev, Battulga Munkhbat, Filipp Komissarenko, Marius Franckevicius, Anvar A Zakhidov, Timur Shegai, Yuri S Kivshar, and Sergey V Makarov. Tunable hybrid fano resonances in halide perovskite nanoparticles. Nano letters, 18(9):5522-5529, 2018.

[40] Aleksandr Vaskin, Justus Bohn, Katie E Chong, Tobias Bucher, Matthias Zilk, Duk-Yong Choi, Dragomir N Neshev, Yuri S Kivshar, Thomas Pertsch, and Isabelle Staude. Directional and spectral shaping of light emission with mie-resonant silicon nanoantenna arrays. Acs Photonics, 5(4):1359-1364, 2018.

[41] Sheng Liu, Aleksandr Vaskin, Sadhvikas Addamane, Benjamin Leung, Miao-Chan Tsai, Yuanmu Yang, Polina P Vabishchevich, Gordon A Keeler, George Wang, Xiaowei He, et al. Light-emitting metasurfaces: simultaneous control of spontaneous emission and far-field radiation. Nano letters, 18(11):6906-6914, 2018.

[42] Aleksandr Vaskin, Radoslaw Kolkowski, A Femius Koenderink, and Isabelle Staude. Light-emitting metasurfaces. Nanophotonics, 8(7):11511198, 2019.

[43] Isabelle Staude, Thomas Pertsch, and Yuri S Kivshar. All-dielectric resonant meta-optics lightens up. ACS Photonics, 6(4):802-814, 2019.

[44] Tobias Bucher, Aleksandr Vaskin, Rajeshkumar Mupparapu, Franz JF Löchner, Antony George, Katie E Chong, Stefan Fasold, Christof Neumann, Duk-Yong Choi, Falk Eilenberger, et al. Tailoring photoluminescence from mos2 monolayers by mie-resonant metasurfaces. ACS Photonics, 6(4):1002-1009, 2019.

[45] Son Tung Ha, Yuan Hsing Fu, Naresh Kumar Emani, Zhenying Pan, Reuben M Bakker, Ramón Paniagua-Domínguez, and Arseniy I Kuznetsov. Directional lasing in resonant semiconductor nanoantenna arrays. Nature nanotechnology, 13(11):1042-1047, 2018. 
[46] Ren-Min Ma and Rupert F Oulton. Applications of nanolasers. Nature Nanotechnology, 14(1):12-22, 2019.

[47] Ramón Paniagua-Domínguez, Son Tung Ha, and Arseniy I Kuznetsov. Active and tunable nanophotonics with dielectric nanoantennas. Proceedings of the IEEE, 108(5):749-771, 2019.

[48] Thanh Xuan Hoang, Son Tung Ha, Zhenying Pan, Wee Kee Phua, Ramon Paniagua-Dominguez, Ching Eng Png, Hong-Son Chu, and Arseniy I Kuznetsov. Collective mie resonances for directional on-chip nanolasers. Nano Letters, 20(8):5655-5661, 2020.

[49] Mengfei Wu, Son Tung Ha, Sushant Shendre, Emek G Durmusoglu, Weon-Kyu Koh, Diego R Abujetas, José A Sánchez-Gil, Ramon Paniagua-Dominguez, Hilmi Volkan Demir, and Arseniy I Kuznetsov. Room-temperature lasing in colloidal nanoplatelets via mie-resonant bound states in the continuum. Nano Letters, 20(8):6005-6011, 2020.

[50] Ekaterina Tiguntseva, Kirill Koshelev, Alexandra Furasova, Pavel Tonkaev, Vladimir Mikhailovskii, Elena V Ushakova, Denis G Baranov, Timur Shegai, Anvar A Zakhidov, Yuri Kivshar, et al. Roomtemperature lasing from mie-resonant non-plasmonic nanoparticles. ACS Nano, 2020.

[51] Glen W Walker, Vikram C Sundar, Christina M Rudzinski, Aetna W Wun, Moungi G Bawendi, and Daniel G Nocera. Quantum-dot optical temperature probes. Applied Physics Letters, 83(17):3555-3557, 2003.

[52] Son-Tung Ha, Chao Shen, Jun Zhang, and Qihua Xiong. Laser cooling of organic-inorganic lead halide perovskites. Nature Photonics, 10(2):115, 2016.

[53] M Balkanski, RF Wallis, and E Haro. Anharmonic effects in light scattering due to optical phonons in silicon. Physical Review B, 28(4):1928, 1983.

[54] I Rodriguez, Lei Shi, Xiaotang Lu, BA Korgel, RA Alvarez-Puebla, and F Meseguer. Silicon nanoparticles as raman scattering enhancers. Nanoscale, 6(11):5666-5670, 2014. 
[55] George P Zograf, Mihail I Petrov, Dmitry A Zuev, Pavel A Dmitriev, Valentin A Milichko, Sergey V Makarov, and Pavel A Belov. Resonant nonplasmonic nanoparticles for efficient temperature-feedback optical heating. Nano Letters, 17(5):2945-2952, 2017.

[56] Nicolo Bontempi, Katie E Chong, Henry W Orton, Isabelle Staude, Duk-Yong Choi, Ivano Alessandri, Yuri S Kivshar, and Dragomir N Neshev. Highly sensitive biosensors based on all-dielectric nanoresonators. Nanoscale, 9(15):4972-4980, 2017.

[57] Ozlem Yavas, Mikael Svedendahl, Paulina Dobosz, Vanesa Sanz, and Romain Quidant. On-a-chip biosensing based on all-dielectric nanoresonators. Nano letters, 17(7):4421-4426, 2017.

[58] Andreas Tittl, Aleksandrs Leitis, Mingkai Liu, Filiz Yesilkoy, DukYong Choi, Dragomir N Neshev, Yuri S Kivshar, and Hatice Altug. Imaging-based molecular barcoding with pixelated dielectric metasurfaces. Science, 360(6393):1105-1109, 2018.

[59] E Mitsai, A Kuchmizhak, E Pustovalov, A Sergeev, A Mironenko, S Bratskaya, DP Linklater, A Balčytis, E Ivanova, and S Juodkazis. Chemically non-perturbing sers detection of a catalytic reaction with black silicon. Nanoscale, 10(20):9780-9787, 2018.

[60] Ozlem Yavas, Mikael Svedendahl, and Romain Quidant. Unravelling the role of electric and magnetic dipoles in biosensing with si nanoresonators. ACS nano, 13(4):4582-4588, 2019.

[61] Filiz Yesilkoy, Eduardo R Arvelo, Yasaman Jahani, Mingkai Liu, Andreas Tittl, Volkan Cevher, Yuri Kivshar, and Hatice Altug. Ultrasensitive hyperspectral imaging and biodetection enabled by dielectric metasurfaces. Nature Photonics, 13(6):390-396, 2019.

[62] Jose Garcia-Guirado, Mikael Svedendahl, Joaquim Puigdollers, and Romain Quidant. Enhanced chiral sensing with dielectric nanoresonators. Nano letters, 20(1):585-591, 2019.

[63] Maxim R Shcherbakov, Dragomir N Neshev, Ben Hopkins, Alexander S Shorokhov, Isabelle Staude, Elizaveta V Melik-Gaykazyan, Manuel Decker, Alexander A Ezhov, Andrey E Miroshnichenko, and Igal 
Brener. Enhanced third-harmonic generation in silicon nanoparticles driven by magnetic response. Nano Letters, 14(11):6488-6492, 2014.

[64] Daria Smirnova and Yuri S Kivshar. Multipolar nonlinear nanophotonics. Optica, 3(11):1241-1255, 2016.

[65] Guixin Li, Shuang Zhang, and Thomas Zentgraf. Nonlinear photonic metasurfaces. Nature Reviews Materials, 2(5):1-14, 2017.

[66] Gustavo Grinblat, Yi Li, Michael P Nielsen, Rupert F Oulton, and Stefan A Maier. Efficient third harmonic generation and nonlinear subwavelength imaging at a higher-order anapole mode in a single germanium nanodisk. ACS nano, 11(1):953-960, 2017.

[67] Sergey V Makarov, Mihail I Petrov, Urs Zywietz, Valentin Milichko, Dmitry Zuev, Natalia Lopanitsyna, Alexey Kuksin, Ivan Mukhin, George Zograf, and Evgeniy Ubyivovk. Efficient second-harmonic generation in nanocrystalline silicon nanoparticles. Nano Letters, 17(5):3047-3053, 2017.

[68] Sheng Liu, Polina P Vabishchevich, Aleksandr Vaskin, John L Reno, Gordon A Keeler, Michael B Sinclair, Isabelle Staude, and Igal Brener. An all-dielectric metasurface as a broadband optical frequency mixer. Nature communications, 9(1):2507, 2018.

[69] Sergey Kruk, Alexander Poddubny, Daria Smirnova, Lei Wang, Alexey Slobozhanyuk, Alexander Shorokhov, Ivan Kravchenko, Barry LutherDavies, and Yuri Kivshar. Nonlinear light generation in topological nanostructures. Nature nanotechnology, 14(2):126-130, 2019.

[70] Lei Xu, Grégoire Saerens, Maria Timofeeva, Daria A Smirnova, Irina Volkovskaya, Mykhaylo Lysevych, Rocio Camacho-Morales, Marcus Cai, Khosro Zangeneh Kamali, Lujun Huang, et al. Forward and backward switching of nonlinear unidirectional emission from gaas nanoantennas. ACS nano, 14(2):1379-1389, 2019.

[71] Basudeb Sain, Cedrik Meier, and Thomas Zentgraf. Nonlinear optics in all-dielectric nanoantennas and metasurfaces: a review. Advanced Photonics, 1(2):024002, 2019. 
[72] Kirill Koshelev, Sergey Kruk, Elizaveta Melik-Gaykazyan, Jae-Hyuck Choi, Andrey Bogdanov, Hong-Gyu Park, and Yuri Kivshar. Subwavelength dielectric resonators for nonlinear nanophotonics. Science, 367(6475):288-292, 2020.

[73] Aleksandra Furasova, Emanuele Calabró, Enrico Lamanna, Ekaterina Tiguntseva, Elena Ushakova, Eugene Ubyivovk, Vladimir Mikhailovskii, Anvar Zakhidov, Sergey Makarov, and Aldo Di Carlo. Resonant silicon nanoparticles for enhanced light harvesting in halide perovskite solar cells. Advanced Optical Materials, 6(21):1800576, 2018.

[74] David Boyer, Philippe Tamarat, Abdelhamid Maali, Brahim Lounis, and Michel Orrit. Photothermal imaging of nanometer-sized metal particles among scatterers. Science, 297(5584):1160-1163, 2002.

[75] Gereon Huttmann and Reginald Birngruber. On the possibility of high-precision photothermal microeffects and the measurement of fast thermal denaturation of proteins. IEEE Journal of selected topics in quantum electronics, 5(4):954-962, 1999.

[76] Guillaume Baffou and Romain Quidant. Thermo-plasmonics: using metallic nanostructures as nano-sources of heat. Laser $\&$ Photonics Reviews, 7(2):171-187, 2013.

[77] SI Anisimov, BL Kapeliovich, TL Perelman, et al. Electron emission from metal surfaces exposed to ultrashort laser pulses. Zh. Eksp. Teor. Fiz, 66(2):375-377, 1974.

[78] Ellen J Yoffa. Screening of hot-carrier relaxation in highly photoexcited semiconductors. Physical Review B, 23(4):1909, 1981.

[79] Theodore Sjodin, Hrvoje Petek, and Hai-Lung Dai. Ultrafast carrier dynamics in silicon: A two-color transient reflection grating study on a (111) surface. Physical review letters, 81(25):5664, 1998.

[80] Marco Bernardi, Derek Vigil-Fowler, Chin Shen Ong, Jeffrey B Neaton, and Steven G Louie. Ab initio study of hot electrons in gaas. Proceedings of the National Academy of Sciences, 112(17):5291-5296, 2015. 
[81] Sergey Makarov, Lada Kolotova, Sergey Starikov, Urs Zywietz, and Boris Chichkov. Resonant silicon nanoparticles with controllable crystalline states and nonlinear optical responses. Nanoscale, 10(24):1140311409, 2018.

[82] AO Larin, A Nominé, EI Ageev, J Ghanbaja, LN Kolotova, SV Starikov, S Bruyère, T Belmonte, SV Makarov, and DA Zuev. Plasmonic nanosponges filled with silicon for enhanced white light emission. Nanoscale, 12(2):1013-1021, 2020.

[83] Lauren L Taylor, Ryan E Scott, and Jie Qiao. Integrating twotemperature and classical heat accumulation models to predict femtosecond laser processing of silicon. Optical Materials Express, 8(3):648-658, 2018.

[84] Gustav Mie. Beiträge zur optik trüber medien, speziell kolloidaler metallösungen. Annalen der Physik, 330(3):377-445, 1908.

[85] Craig F Bohren and Donald R Huffman. Absorption and scattering of light by small particles. John Wiley \& Sons, 2008.

[86] Michael I Tribelsky. Anomalous light absorption by small particles. EPL (Europhysics Letters), 94(1):14004, 2011.

[87] Andrey E Miroshnichenko and Michael I Tribelsky. Ultimate absorption in light scattering by a finite obstacle. Physical review letters, 120(3):033902, 2018.

[88] George P Zograf, Mihail I Petrov, Dmitry A Zuev, Pavel A Dmitriev, Valentin A Milichko, Sergey V Makarov, and Pavel A Belov. Resonant nonplasmonic nanoparticles for efficient temperature-feedback optical heating. Nano Lett., 17(5):2945-2952, 2017.

[89] Edward D Palik. Handbook of optical constants of solids, volume 3. Academic press, 1998.

[90] Gang Chen. Phonon heat conduction in nanostructures. International journal of thermal sciences, 39(4):471-480, 2000.

[91] Joao Cunha, Tian-Long Guo, Giuseppe Della Valle, Alemayehu Nana Koya, Remo Proietti Zaccaria, and Alessandro Alabastri. Controlling 
light, heat, and vibrations in plasmonics and phononics. Advanced Optical Materials, 8(24):2001225, 2020.

[92] G Baffou, R Quidant, and Ch Girard. Heat generation in plasmonic nanostructures: Influence of morphology. Applied Physics Letters, 94(15):153109, 2009.

[93] Stefano Danesi and Ivano Alessandri. Using optical resonances to control heat generation and propagation in silicon nanostructures. arXiv preprint arXiv:1811.10908, 2018.

[94] Stefano Danesi, Marco Gandolfi, Luca Carletti, Nicolò Bontempi, Costantino De Angelis, Francesco Banfi, and Ivano Alessandri. Photoinduced heat generation in non-plasmonic nanoantennas. Physical Chemistry Chemical Physics, 20(22):15307-15315, 2018.

[95] Quinten A Akkerman, Valerio D'Innocenzo, Sara Accornero, Alice Scarpellini, Annamaria Petrozza, Mirko Prato, and Liberato Manna. Tuning the optical properties of cesium lead halide perovskite nanocrystals by anion exchange reactions. Journal of the American Chemical Society, 137(32):10276-10281, 2015.

[96] Steven C Erwin, Lijun Zu, Michael I Haftel, Alexander L Efros, Thomas A Kennedy, and David J Norris. Doping semiconductor nanocrystals. Nature, 436(7047):91, 2005.

[97] WE Spear and PG Le Comber. Substitutional doping of amorphous silicon. Solid state communications, 17(9):1193-1196, 1975.

[98] Tomer Lewi, Prasad P Iyer, Nikita A Butakov, Alexander A Mikhailovsky, and Jon A Schuller. Widely tunable infrared antennas using free carrier refraction. Nano letters, 15(12):8188-8193, 2015.

[99] Charles Kittel and Paul McEuen. Introduction to solid state physics, volume 8. Wiley New York, 1976.

[100] Robert W Boyd. Nonlinear optics. Academic press, 2020.

[101] Denis G Baranov, Sergey V Makarov, Valentin A Milichko, Sergey I Kudryashov, Alexander E Krasnok, and Pavel A Belov. Nonlinear transient dynamics of photoexcited resonant silicon nanostructures. Acs Photonics, 3(9):1546-1551, 2016. 
[102] Ying Li, Wei Li, Tiancheng Han, Xu Zheng, Jiaxin Li, Baowen Li, Shanhui Fan, and Cheng-Wei Qiu. Transforming heat transfer with thermal metamaterials and devices. Nature Reviews Materials, pages $1-20,2021$.

[103] RW Powell, Cho Yen Ho, and Peter Edward Liley. Thermal conductivity of selected materials, volume 8. US Department of Commerce, National Bureau of Standards Washington, DC, 1966.

[104] HR Shanks, PD Maycock, PH Sidles, and GC Danielson. Thermal conductivity of silicon from 300 to 1400 k. Physical Review, 130(5):1743, 1963.

[105] David G Cahill. Thermal conductivity measurement from 30 to $750 \mathrm{k}$ : the $3 \omega$ method. Review of scientific instruments, 61(2):802-808, 1990.

[106] Karl Stephan and A Laesecke. The thermal conductivity of fluid air. Journal of physical and chemical reference data, 14(1):227-234, 1985.

[107] Yoichi Takahashi and Hidetoshi Akiyama. Heat capacity of gold from 80 to $1000 \mathrm{k}$. Thermochimica acta, 109(1):105-109, 1986.

[108] AS Okhotin, A Pushkarskii, and V Gorbachev. Thermophysical properties of semiconductors; atom publ. House, Moscow, 1972.

[109] Malcom W Chase Jr and NIST-JANAF Thermochemical Tables. Data reported in nist standard reference database 69, june 2005 release: Nist chemistry webbook. J. Phys. Chem. Ref. Data, Monograph, 9:1-1951, 1998.

[110] Joseph Hilsenrath. Tables of thermal properties of gases: comprising tables of thermodynamic and transport properties of air, argon, carbon dioxide, carbon monoxide, hydrogen, nitrogen, oxygen, and steam, volume 564. US Department of Commerce, National Bureau of Standards, 1955 .

[111] Dong-Kwon Lim, Ki-Seok Jeon, Hyung Min Kim, Jwa-Min Nam, and Yung Doug Suh. Nanogap-engineerable raman-active nanodumbbells for single-molecule detection. Nature materials, 9(1):60-67, 2010. 
[112] Reuben M Bakker, Dmitry Permyakov, Ye Feng Yu, Dmitry Markovich, Ramón Paniagua-Domínguez, Leonard Gonzaga, Anton Samusev, Yuri Kivshar, Boris Luk'yanchuk, and Arseniy I Kuznetsov. Magnetic and electric hotspots with silicon nanodimers. Nano Letters, 15(3):2137$2142,2015$.

[113] Martín Caldarola, Pablo Albella, Emiliano Cortés, Mohsen Rahmani, Tyler Roschuk, Gustavo Grinblat, Rupert F Oulton, Andrea V Bragas, and Stefan A Maier. Non-plasmonic nanoantennas for surface enhanced spectroscopies with ultra-low heat conversion. Nature communications, 6:7915, 2015.

[114] Jwa-Min Nam, Jeong-Wook Oh, Haemi Lee, and Yung Doug Suh. Plasmonic nanogap-enhanced raman scattering with nanoparticles. Accounts of chemical research, 49(12):2746-2755, 2016.

[115] Gediminas Gervinskas, Gediminas Seniutinas, Jennifer S Hartley, Sasikaran Kandasamy, Paul R Stoddart, Narges F Fahim, and Saulius Juodkazis. Surface-enhanced raman scattering sensing on black silicon. Annalen der Physik, 525(12):907-914, 2013.

[116] Andrey Lagarkov, Irina Boginskaya, Igor Bykov, Igor Budashov, Andrey Ivanov, Ilya Kurochkin, Ilya Ryzhikov, Ilya Rodionov, Marina Sedova, Alexander Zverev, et al. Light localization and sers in tip-shaped silicon metasurface. Optics express, 25(15):17021-17038, 2017.

[117] Carlos DS Brites, Patricia P Lima, Nuno JO Silva, Angel Millán, Vitor S Amaral, Fernando Palacio, and Luís D Carlos. Thermometry at the nanoscale. Nanoscale, 4(16):4799-4829, 2012.

[118] Carlo Bradac, Shuang Fang Lim, Huan-Cheng Chang, and Igor Aharonovich. Optical nanoscale thermometry: From fundamental mechanisms to emerging practical applications. Advanced Optical Materials, page 2000183, 2020.

[119] Yuuki Hagiwara, Kohei Takahata, Junji Torimoto, and Junji Yamamoto. Co2 raman thermometer improvement: Comparing hot band and stokes and anti-stokes raman scattering thermometers. Journal of Raman Spectroscopy, 49(11):1776-1781, 2018. 
[120] Pawel Karpinski, Steven Jones, Hana Š́́pová-Jungová, Ruggero Verre, and Mikael Käll. Optical rotation and thermometry of laser tweezed silicon nanorods. Nano Letters, 2020.

[121] TR Hart, RL Aggarwal, and Benjamin Lax. Temperature dependence of raman scattering in silicon. Physical Review B, 1(2):638, 1970.

[122] Pavel A Dmitriev, Denis G Baranov, Valentin A Milichko, Sergey V Makarov, Ivan S Mukhin, Anton K Samusev, Alexander E Krasnok, Pavel A Belov, and Yuri S Kivshar. Resonant raman scattering from silicon nanoparticles enhanced by magnetic response. Nanoscale, 8(18):9721-9726, 2016.

[123] Ivano Alessandri and John R Lombardi. Enhanced raman scattering with dielectrics. Chemical reviews, 116(24):14921-14981, 2016.

[124] Denis G Baranov, Ruggero Verre, Pawel Karpinski, and Mikael Källl. Anapole-enhanced intrinsic raman scattering from silicon nanodisks. ACS Photonics, 5(7):2730-2736, 2018.

[125] George P Zograf, Daniil Ryabov, Viktoria Rutckaia, Pavel Voroshilov, Pavel Tonkaev, Dmitry V Permyakov, Yuri Kivshar, and Sergey V Makarov. Stimulated raman scattering from mie-resonant subwavelength nanoparticles. Nano Letters, 20(8):5786-5791, 2020.

[126] Søren Raza and Anders Kristensen. Raman scattering in highrefractive-index nanostructures. Nanophotonics, 1(ahead-of-print), 2020 .

[127] S Rudin, TL Reinecke, and B Segall. Temperature-dependent exciton linewidths in semiconductors. Physical Review B, 42(17):11218, 1990.

[128] Jiahao Yan, Yuchao Li, Zaizhu Lou, Churong Ma, Guowei Yang, and Baojun Li. Active tuning of mie resonances to realize sensitive photothermal measurement of single nanoparticles. Materials Horizons, $7(6): 1542-1551,2020$.

[129] Yi-Shiou Duh, Yusuke Nagasaki, Yu-Lung Tang, Pang-Han Wu, HaoYu Cheng, Te-Hsin Yen, Hou-Xian Ding, Kentaro Nishida, Ikuto Hotta, Jhen-Hong Yang, et al. Giant photothermal nonlinearity in a single silicon nanostructure. Nature Communications, 11(1):1-9, 2020. 
[130] Yonatan Sivan and Shi-Wei Chu. Nonlinear plasmonics at high temperatures. Nanophotonics, 6(1):317-328, 2017.

[131] Vasilii Mylnikov, Son Tung Ha, Zhenying Pan, Vytautas Valuckas, Ramón Paniagua-Domínguez, Hilmi Volkan Demir, and Arseniy I Kuznetsov. Lasing action in single subwavelength particles supporting supercavity modes. ACS Nano, 2020.

[132] Vineet Kumar Rai. Temperature sensors and optical sensors. Applied Physics B, 88(2):297-303, 2007.

[133] M Aouassa, E Mitsai, S Syubaev, D Pavlov, A Zhizhchenko, I Jadli, L Hassayoun, G Zograf, S Makarov, and A Kuchmizhak. Temperaturefeedback direct laser reshaping of silicon nanostructures. Applied Physics Letters, 111(24):243103, 2017.

[134] Valentin A Milichko, Dmitry A Zuev, Denis G Baranov, George P Zograf, Katerina Volodina, Andrei A Krasilin, Ivan S Mukhin, Pavel A Dmitriev, and Vladimir V Vinogradov. Metal-dielectric nanocavity for real-time tracing molecular events with temperature feedback. Laser \& Photonics Reviews, 12(1), 2018.

[135] Nils Odebo Länk, Peter Johansson, and Mikael Källl. Optical tweezing and photothermal properties of resonant dielectric and metallic nanospheres. ACS Photonics, 2020.

[136] Muhammet Erkan Köse, Bruce F Carroll, and Kirk S Schanze. Preparation and spectroscopic properties of multiluminophore luminescent oxygen and temperature sensor films. Langmuir, 21(20):9121-9129, 2005.

[137] Kalman B Migler and Anthony J Bur. Fluorescence based measurement of temperature profiles during polymer processing. Polymer Engineering $\&$ Science, 38(1):213-221, 1998.

[138] David Ross, Michael Gaitan, and Laurie E Locascio. Temperature measurement in microfluidic systems using a temperature-dependent fluorescent dye. Analytical chemistry, 73(17):4117-4123, 2001. 
[139] G Baffou, MP Kreuzer, F Kulzer, and R Quidant. Temperature mapping near plasmonic nanostructures using fluorescence polarization anisotropy. Optics express, 17(5):3291-3298, 2009.

[140] Jiao Feng, Kaijun Tian, Dehui Hu, Shuangqing Wang, Shayu Li, Yi Zeng, Yi Li, and Guoqiang Yang. A triarylboron-based fluorescent thermometer: Sensitive over a wide temperature range. Angewandte Chemie, 123(35):8222-8226, 2011.

[141] Sha Li, Kai Zhang, Jui-Ming Yang, Liwei Lin, and Haw Yang. Single quantum dots as local temperature markers. Nano letters, 7(10):31023105, 2007.

[142] Pedro Jorge, Manuel António Martins, Tito Trindade, José Luís Santos, and Faramarz Farahi. Optical fiber sensing using quantum dots. Sensors, 7(12):3489-3534, 2007.

[143] Vladimir A Vlaskin, Nils Janssen, Jos van Rijssel, Rémi Beaulac, and Daniel R Gamelin. Tunable dual emission in doped semiconductor nanocrystals. Nano letters, 10(9):3670-3674, 2010.

[144] P Haro-González, L Martínez-Maestro, IR Martín, J García-Solé, and D Jaque. High-sensitivity fluorescence lifetime thermal sensing based on cdte quantum dots. small, 8(17):2652-2658, 2012.

[145] Ning-Ning Dong, Marco Pedroni, Fabio Piccinelli, Giamaica Conti, Andrea Sbarbati, Juan Enrique Ramírez-Hernández, Laura Martínez Maestro, Maria Carmen Iglesias-de la Cruz, Francisco Sanz-Rodriguez, Angeles Juarranz, et al. Nir-to-nir two-photon excited caf2: Tm3+, yb3+ nanoparticles: multifunctional nanoprobes for highly penetrating fluorescence bio-imaging. ACS nano, 5(11):8665-8671, 2011.

[146] Fiorenzo Vetrone, Rafik Naccache, Alicia Zamarrón, Angeles Juarranz de la Fuente, Francisco Sanz-Rodríguez, Laura Martinez Maestro, Emma Martin Rodriguez, Daniel Jaque, Jose Garcia Sole, and John A Capobianco. Temperature sensing using fluorescent nanothermometers. ACS nano, 4(6):3254-3258, 2010.

[147] Xingjun Zhu, Wei Feng, Jian Chang, Yan-Wen Tan, Jiachang Li, Min Chen, Yun Sun, and Fuyou Li. Temperature-feedback upconversion 
nanocomposite for accurate photothermal therapy at facile temperature. Nature communications, 7(1):1-10, 2016.

[148] Xin Wang, Xianggui Kong, Yi Yu, Yajuan Sun, and Hong Zhang. Effect of annealing on upconversion luminescence of zno: Er3+ nanocrystals and high thermal sensitivity. The Journal of Physical Chemistry C, 111(41):15119-15124, 2007.

[149] Kory Green, Kai Huang, Hai Pan, Gang Han, and Shuang Fang Lim. Optical temperature sensing with infrared excited upconversion nanoparticles. Frontiers in chemistry, 6:416, 2018.

[150] Taras Plakhotnik, Haroon Aman, and Huan-Cheng Chang. All-optical single-nanoparticle ratiometric thermometry with a noise floor of $0.3 \mathrm{k}$ hz- 1/2. Nanotechnology, 26(24):245501, 2015.

[151] Jing-Wei Fan, Ivan Cojocaru, Joe Becker, Ilya V Fedotov, Masfer Hassan A Alkahtani, Abdulrahman Alajlan, Sean Blakley, Mohammadreza Rezaee, Anna Lyamkina, Yuri N Palyanov, et al. Germanium-vacancy color center in diamond as a temperature sensor. ACS Photonics, 5(3):765-770, 2018.

[152] Christian T Nguyen, Ruffin E Evans, Alp Sipahigil, Mihir K Bhaskar, Denis D Sukachev, Viatcheslav N Agafonov, Valery A Davydov, Liudmila F Kulikova, Fedor Jelezko, and Mikhail D Lukin. All-optical nanoscale thermometry with silicon-vacancy centers in diamond. $A p$ plied Physics Letters, 112(20):203102, 2018.

[153] Masfer Alkahtani, Ivan Cojocaru, Xiaohan Liu, Tobias Herzig, Jan Meijer, Johannes Küpper, Tobias Lühmann, Alexey V Akimov, and Philip R Hemmer. Tin-vacancy in diamonds for luminescent thermometry. Applied Physics Letters, 112(24):241902, 2018.

[154] Toan Trong Tran, Blake Regan, Evgeny A Ekimov, Zhao Mu, Yu Zhou, Wei-bo Gao, Prineha Narang, Alexander S Solntsev, Milos Toth, Igor Aharonovich, et al. Anti-stokes excitation of solid-state quantum emitters for nanoscale thermometry. Science advances, 5(5):eaav9180, 2019.

[155] Ph Buffat and Jean Pierre Borel. Size effect on the melting temperature of gold particles. Physical review A, 13(6):2287, 1976. 
[156] RJ Nemanich, DK Biegelsen, RA Street, and LE Fennell. Raman scattering from solid silicon at the melting temperature. Physical Review B, 29(10):6005, 1984.

[157] IV Talyzin, MV Samsonov, VM Samsonov, M Yu Pushkar, and VV Dronnikov. Size dependence of the melting point of silicon nanoparticles: molecular dynamics and thermodynamic simulation. Semiconductors, 53(7):947-953, 2019.

[158] H Scheel, S Khachadorian, M Cantoro, A Colli, AC Ferrari, and $\mathrm{C}$ Thomsen. Silicon nanowire optical raman line shapes at cryogenic and elevated temperatures. physica status solidi (b), 245(10):20902093, 2008.

[159] George P Zograf, Alexander S Timin, Albert P Muslimov, Ivan I Shishkin, Alexandre Nomine, Jaafar Ghanbaja, Pintu Ghosh, Qiang Li, Mikhail V Zyuzin, and Sergey V Makarov. All-optical nanoscale heating and thermometry with resonant dielectric nanoparticles for photoinduced tumor treatment. arXiv preprint arXiv:1906.06150, 2019.

[160] DR Lide. Handbook of chemistry and physics, 81st edn., sect. 11, 2000.

[161] Sadao Adachi. Properties of semiconductor alloys: group-IV, III-V and II-VI semiconductors, volume 28. John Wiley \& Sons, 2009.

[162] Gorachand Ghosh. Handbook of optical constants of solids: Handbook of thermo-optic coefficients of optical materials with applications. Academic Press, 1998.

[163] MSYPM Levinshtein. Handbook series on semiconductor parameters, volume 1. World Scientific, 1997.

[164] KH Tsang, HW Kui, and KP Chik. Calorimetric studies of the heat capacity and relaxation of amorphous si prepared by electron beam evaporation. Journal of applied physics, 74(8):4932-4935, 1993.

[165] BL Zink, R Pietri, and F Hellman. Thermal conductivity and specific heat of thin-film amorphous silicon. Physical review letters, 96(5):055902, 2006. 
[166] Oguz Yavas, Nhan Do, Andrew C Tam, PT Leung, Wing P Leung, Hee K Park, Costas P Grigoropoulos, Johannes Boneberg, and Paul Leiderer. Temperature dependence of optical properties for amorphous silicon at wavelengths of 632.8 and $752 \mathrm{~nm}$. Optics letters, 18(7):540$542,1993$.

[167] K Takimoto, A Fukuta, Y Yamamoto, N Yoshida, T Itoh, and S Nonomura. Linear thermal expansion coefficients of amorphous and microcrystalline silicon films. Journal of non-crystalline solids, 299:314-317, 2002.

[168] AA El-Sharkawy, AM Abou El-Azm, MI Kenawy, AS Hillal, and HM Abu-Basha. Thermophysical properties of polycrystalline pbs, pbse, and pbte in the temperature range $300-700 \mathrm{k}$. International journal of thermophysics, 4(3):261-269, 1983.

[169] Tomer Lewi, Nikita A Butakov, Hayden A Evans, Mark W Knight, Prasad P Iyer, David Higgs, Hamid Chorsi, Juan Trastoy, Javier Del Valle Granda, Ilya Valmianski, et al. Thermally reconfigurable meta-optics. IEEE Photonics Journal, 11(2):1-16, 2019.

[170] Jonathan M Skelton, Stephen C Parker, Atsushi Togo, Isao Tanaka, and Aron Walsh. Thermal physics of the lead chalcogenides pbs, pbse, and pbte from first principles. Physical Review B, 89(20):205203, 2014.

[171] L Balde, B Legendre, and A Balkhi. Etude du diagramme d'equilibre entre phases du systeme ternaire germanium-etain-tellure. Journal of alloys and compounds, 216(2):285-293, 1995.

[172] Eduardo Morales-Sanchez, EF Prokhorov, J Gonzalez-Hernandez, and A Mendoza-Galvan. Structural, electric and kinetic parameters of ternary alloys of gesbte. Thin Solid Films, 471(1-2):243-247, 2005.

[173] J-L Battaglia, Andrzej Kusiak, Vincent Schick, Andrea Cappella, Claudia Wiemer, Massimo Longo, and Enrico Varesi. Thermal characterization of the si o 2-ge $2 \mathrm{sb} 2$ te 5 interface from room temperature up to 400 c. Journal of Applied Physics, 107(4):044314, 2010.

[174] Ho-Ki Lyeo, David G Cahill, Bong-Sub Lee, John R Abelson, MinHo Kwon, Ki-Bum Kim, Stephen G Bishop, and Byung-ki Cheong. 
Thermal conductivity of phase-change material ge 2 sb 2 te 5 . Applied Physics Letters, 89(15):151904, 2006.

[175] Matthias Stegmaier, Carlos Rıs, Harish Bhaskaran, and Wolfram HP Pernice. Thermo-optical effect in phase-change nanophotonics. Acs Photonics, 3(5):828-835, 2016.

[176] Chao-An Jong, Weileung Fang, Chain-Ming Lee, and Tsung-Shune Chin. Mechanical properties of phase-change recording media: Gesbte films. Japanese Journal of Applied Physics, 40(5R):3320, 2001.

[177] Bruno Brunetti, Carmen Cavallo, Andrea Ciccioli, Guido Gigli, and Alessandro Latini. On the thermal and thermodynamic (in) stability of methylammonium lead halide perovskites. Scientific reports, 6:31896, 2016.

[178] Taketo Handa, Hirokazu Tahara, Tomoko Aharen, and Yoshihiko Kanemitsu. Large negative thermo-optic coefficients of a lead halide perovskite. Science advances, 5(7):eaax0786, 2019.

[179] Chunyu Ge, Mingyu Hu, Peng Wu, Qi Tan, Zhizhong Chen, Yiping Wang, Jian Shi, and Jing Feng. Ultralow thermal conductivity and ultrahigh thermal expansion of single-crystal organic-inorganic hybrid perovskite ch3nh3pbx3 $(\mathrm{x}=\mathrm{cl}, \mathrm{br}, \mathrm{i})$. The Journal of Physical Chemistry C, 122(28):15973-15978, 2018.

[180] Leila Sarkhosh, Hoda Aleali, Rouhollah Karimzadeh, and Nastaran Mansour. Large thermally induced nonlinear refraction of gold nanoparticles stabilized by cyclohexanone. physica status solidi (a), 207(10):2303-2310, 2010.

[181] FC Nix and D MacNair. The thermal expansion of pure metals: copper, gold, aluminum, nickel, and iron. Physical Review, 60(8):597, 1941.

[182] R Karimzadeh and N Mansour. The effect of concentration on the thermo-optical properties of colloidal silver nanoparticles. Optics \&6 Laser Technology, 42(5):783-789, 2010.

[183] T Favaloro, J-H Bahk, and A Shakouri. Characterization of the temperature dependence of the thermoreflectance coefficient for conductive thin films. Review of Scientific Instruments, 86(2):024903, 2015. 
[184] Pavel Tonkaev, George Zograf, and Sergey Makarov. Optical cooling of lead halide perovskite nanoparticles enhanced by mie resonances. Nanoscale, 11(38):17800-17806, 2019.

[185] Peter Pringsheim. Zwei bemerkungen über den unterschied von lumineszenz-und temperaturstrahlung. Zeitschrift für Physik, 57(1112):739-746, 1929 .

[186] A Kastler. Some suggestions concerning the production and detection by optical means of inequalities in the populations of levels of spatial quantization in atoms. application to the stern and gerlach and magnetic resonance experiments. J. Phys. Radium, 11(11):255-265, 1950.

[187] Richard I Epstein, Melvin I Buchwald, Bradley C Edwards, Timothy R Gosnell, and Carl E Mungan. Observation of laser-induced fluorescent cooling of a solid. Nature, 377(6549):500-503, 1995.

[188] Mansoor Sheik-Bahae and Richard I Epstein. Laser cooling of solids. Laser $\&$ Photonics Reviews, 3(1-2):67-84, 2009.

[189] Galina Nemova and Raman Kashyap. Laser cooling of solids. Reports on Progress in Physics, 73(8):086501, 2010.

[190] Mansoor Sheik-Bahae and Richard I Epstein. Can laser light cool semiconductors? Physical Review Letters, 92(24):247403, 2004.

[191] Shao-Lin Wu, Honglei Chen, Hua-Li Wang, Xiaolan Chen, Hao-Cheng Yang, and Seth B Darling. Solar-driven evaporators for water treatment: challenges and opportunities. Environmental Science: Water Research \& Technology, 7(1):24-39, 2021.

[192] Honglei Chen, Shao-Lin Wu, Hua-Li Wang, Qing-Yun Wu, and HaoCheng Yang. Photothermal devices for sustainable uses beyond desalination. Advanced Energy and Sustainability Research, page 2000056, 2021.

[193] Todd P Otanicar and Jay S Golden. Comparative environmental and economic analysis of conventional and nanofluid solar hot water technologies. Environmental science \&6 technology, 43(15):6082-6087, 2009. 
[194] Satoshi Ishii, Ramu Pasupathi Sugavaneshwar, Kai Chen, Thang Duy Dao, and Tadaaki Nagao. Solar water heating and vaporization with silicon nanoparticles at mie resonances. Optical Materials Express, 6(2):640-648, 2016.

[195] Satoshi Ishii, Kai Chen, Hideo Okuyama, and Tadaaki Nagao. Resonant optical absorption and photothermal process in high refractive index germanium nanoparticles. Advanced Optical Materials, $5(5): 1600902,2017$.

[196] Churong Ma, Jiahao Yan, Yingcong Huang, Chengxin Wang, and Guowei Yang. The optical duality of tellurium nanoparticles for broadband solar energy harvesting and efficient photothermal conversion. Science advances, 4(8):eaas9894, 2018.

[197] Bernard L Cohen. Anomalous behavior of tellurium abundances. Geochimica et Cosmochimica Acta, 48(1):203-205, 1984.

[198] SO Gurbatov, E Modin, V Puzikov, P Tonkaev, D Storozhenko, S Sergeev, N Mintcheva, S Yamaguchi, N Tarasenka, A Chuvilin, et al. Au-decorated black tio _2 produced via laser ablation in liquid. arXiv preprint arXiv:2009.10293, 2020.

[199] Noemi Bosio, Hanna Š́pová Jungová, Nils Odebo Länk, Tomasz J Antosiewicz, Ruggero Verre, and Mikael Källl. Plasmonic versus alldielectric nanoantennas for refractometric sensing: A direct comparison. ACS Photonics, 6(6):1556-1564, 2019.

[200] Elisabet Xifre-Perez, Lei Shi, Umut Tuzer, Roberto Fenollosa, Fernando Ramiro-Manzano, Romain Quidant, and Francisco Meseguer. Mirror-image-induced magnetic modes. ACS nano, 7(1):664-668, 2012.

[201] Tanya Hutter, Fu Min Huang, Stephen R Elliott, and Sumeet Mahajan. Near-field plasmonics of an individual dielectric nanoparticle above a metallic substrate. The Journal of Physical Chemistry C, 117(15):77847790, 2013.

[202] Song-Yuan Ding, Jun Yi, Jian-Feng Li, Bin Ren, De-Yin Wu, Rajapandiyan Panneerselvam, and Zhong-Qun Tian. Nanostructurebased plasmon-enhanced raman spectroscopy for surface analysis of materials. Nature Rev. Mater., 1:16021, 2016. 
[203] Zengli Huang, Jianfeng Wang, Zhenghui Liu, Gengzhao Xu, Yingmin Fan, Haijian Zhong, Bing Cao, Chinhua Wang, and Ke Xu. Strong-field-enhanced spectroscopy in silicon nanoparticle electric and magnetic dipole resonance near a metal surface. J. Phys. Chem. C, 119(50):28127-28135, 2015.

[204] Mohsen Rahmani, Lei Xu, Andrey E Miroshnichenko, Andrei Komar, Rocio Camacho-Morales, Haitao Chen, Yair Zárate, Sergey Kruk, Guoquan Zhang, Dragomir N Neshev, et al. Reversible thermal tuning of all-dielectric metasurfaces. Advanced Functional Materials, 27(31):1700580, 2017.

[205] Tomer Lewi, Hayden A Evans, Nikita A Butakov, and Jon A Schuller. Ultrawide thermo-optic tuning of pbte meta-atoms. Nano letters, 17(6):3940-3945, 2017.

[206] Khosro Zangeneh Kamali, Lei Xu, Jonathan Ward, Kai Wang, Guixin Li, Andrey E Miroshnichenko, Dragomir Neshev, and Mohsen Rahmani. Reversible image contrast manipulation with thermally tunable dielectric metasurfaces. Small, page 1805142, 2019.

[207] Neil W Ashcroft and N. D. Mermin. Solid state physics. Holt, Reinhart and Winston, 1976.

[208] A Esser, K Seibert, H Kurz, GN Parsons, C Wang, BN Davidson, G Lucovsky, and RJ Nemanich. Ultrafast recombination and trapping in amorphous silicon. J. Non-Cryst. Solids, 114:573-575, 1989.

[209] Tomer Lewi, Nikita A Butakov, and Jon A Schuller. Thermal tuning capabilities of semiconductor metasurface resonators. Nanophotonics, 8(2):331-338, 2018.

[210] MC Downer and CV Shank. Ultrafast heating of silicon on sapphire by femtosecond optical pulses. Phys. Rev. Lett., 56(7):761, 1986.

[211] Gustavo Grinblat, Haizhong Zhang, Michael P Nielsen, Leonid Krivitsky, Rodrigo Berté, Yi Li, Benjamin Tilmann, Emiliano Cortés, Rupert F Oulton, Arseniy I Kuznetsov, et al. Efficient ultrafast all-optical modulation in a nonlinear crystalline gallium phosphide nanodisk at the anapole excitation. Science Advances, 6(34):eabb3123, 2020. 
[212] Sergey Makarov, Sergey Kudryashov, Ivan Mukhin, Alexey Mozharov, Valentin Milichko, Alexander Krasnok, and Pavel Belov. Tuning of magnetic optical response in a dielectric nanoparticle by ultrafast photoexcitation of dense electron-hole plasma. Nano letters, 15(9):61876192, 2015.

[213] Maxim R Shcherbakov, Polina P Vabishchevich, Alexander S Shorokhov, Katie E Chong, Duk-Yong Choi, Isabelle Staude, Andrey E Miroshnichenko, Dragomir N Neshev, Andrey A Fedyanin, and Yuri S Kivshar. Ultrafast all-optical switching with magnetic resonances in nonlinear dielectric nanostructures. Nano letters, 15(10):6985-6990, 2015 .

[214] Ted V Tsoulos and Giulia Tagliabue. Self-induced thermo-optical effects in silicon and germanium dielectric nanoresonators. Nanophotonics, 1(ahead-of-print), 2020.

[215] Liyi Hsu and Abdoulaye Ndao. Broadband optical power limiter metalens. arXiv preprint arXiv:2101.01249, 2021.

[216] Tianyue Zhang, Ying Che, Kai Chen, Jian Xu, Yi Xu, Te Wen, Guowei $\mathrm{Lu}$, Xiaowei Liu, Bin Wang, Xiaoxuan Xu, et al. Anapole mediated giant photothermal nonlinearity in nanostructured silicon. Nature communications, 11(1):1-9, 2020.

[217] E Mitsai, M Naffouti, T David, M Abbarchi, L Hassayoun, D Storozhenko, A Mironenko, S Bratskaya, S Juodkazis, S Makarov, et al. Si 1- $\mathrm{x}$ ge $\mathrm{x}$ nanoantennas with a tailored raman response and light-to-heat conversion for advanced sensing applications. Nanoscale, 11(24):11634-11641, 2019.

[218] Urs Zywietz, Andrey B Evlyukhin, Carsten Reinhardt, and Boris N Chichkov. Laser printing of silicon nanoparticles with resonant optical electric and magnetic responses. Nature communications, 5:3402, 2014.

[219] Jingyi Tian, Hao Luo, Yuanqing Yang, Fei Ding, Yurui Qu, Ding Zhao, Min Qiu, and Sergey I Bozhevolnyi. Active control of anapole states by structuring the phase-change alloy ge 2 sb 2 te 5 . Nature communications, 10(1):396, 2019. 
[220] Carlota Ruiz de Galarreta, Ivan Sinev, Arseny M Alexeev, Pavel Trofimov, Konstantin Ladutenko, Santiago Garcia-Cuevas Carrillo, Emanuele Gemo, Anna Baldycheva, Jacopo Bertolotti, and C David Wright. Reconfigurable multilevel control of hybrid alldielectric phase-change metasurfaces. Optica, 2020.

[221] John D Joannopoulos and Gerald Lucovsky, editors. The Physics of Hydrogenated Amorphous Silicon II: Electronic and Vibrational Properties. Springer-Verlag, Berlin, 1984.

[222] Letian Wang, Yoonsoo Rho, Wan Shou, Sukjoon Hong, Kimihiko Kato, Matthew Eliceiri, Meng Shi, Costas P Grigoropoulos, Heng Pan, Carlo Carraro, et al. Programming nanoparticles in multiscale: optically modulated assembly and phase switching of silicon nanoparticle array. ACS nano, 12(3):2231-2241, 2018.

[223] Georgii Petrovich Zograf, Ye Feng Yu, Kseniya Vladimirovna Baryshnikova, Arsenii Igorevich Kuznetsov, and Sergei Vladimirivich Makarov. Local crystallization of a resonant amorphous silicon nanoparticle for the implementation of optical nanothermometry. JETP Letters, 107(11):699-704, 2018.

[224] Dominic Lencer, Martin Salinga, Blazej Grabowski, Tilmann Hickel, Jörg Neugebauer, and Matthias Wuttig. A map for phase-change materials. Nature Mater., 7(12):972-977, 2008.

[225] Kostiantyn Shportko, Stephan Kremers, Michael Woda, Dominic Lencer, John Robertson, and Matthias Wuttig. Resonant bonding in crystalline phase-change materials. Nature Mater., 7(8):653-658, 2008.

[226] Qian Wang, Edward T. F. Rogers, Behrad Gholipour, Chih-Ming Wang, Guanghui Yuan, Jinghua Teng, and Nikolay I. Zheludev. Optically reconfigurable metasurfaces and photonic devices based on phase change materials. Nature Photonics, 10(1):60-65, 2016.

[227] Mikhail V Rybin, Kirill B Samusev, Stanislav Yu Lukashenko, Yuri S Kivshar, and Mikhail F Limonov. Transition from two-dimensional photonic crystals to dielectric metasurfaces in the optical diffraction with a fine structure. Sci. Rep., 6:30773, 2016. 
[228] Yusuke Nagasaki, Masafumi Suzuki, Ikuto Hotta, and Junichi Takahara. Control of si-based all-dielectric printing color through oxidation. ACS Photonics, 5(4):1460-1466, 2018.

[229] Xiaolong Zhu, Wei Yan, Uriel Levy, N Asger Mortensen, and Anders Kristensen. Resonant laser printing of structural colors on high-index dielectric metasurfaces. Science advances, 3(5):e1602487, 2017.

[230] Andrey B Evlyukhin, Carsten Reinhardt, and Boris N Chichkov. Multipole light scattering by nonspherical nanoparticles in the discrete dipole approximation. Physical Review B, 84(23):235429, 2011.

[231] Isabelle Staude, Andrey E Miroshnichenko, Manuel Decker, Nche T Fofang, Sheng Liu, Edward Gonzales, Jason Dominguez, Ting Shan Luk, Dragomir N Neshev, Igal Brener, et al. Tailoring directional scattering through magnetic and electric resonances in subwavelength silicon nanodisks. ACS nano, 7(9):7824-7832, 2013.

[232] Andrey B Evlyukhin, Tim Fischer, Carsten Reinhardt, and Boris N Chichkov. Optical theorem and multipole scattering of light by arbitrarily shaped nanoparticles. Physical Review B, 94(20):205434, 2016.

[233] Pavel D Terekhov, Kseniia V Baryshnikova, Yuriy A Artemyev, Alina Karabchevsky, Alexander S Shalin, and Andrey B Evlyukhin. Multipolar response of nonspherical silicon nanoparticles in the visible and near-infrared spectral ranges. Physical Review B, 96(3):035443, 2017.

[234] M Morita, T Ohmi, E Hasegawa, M Kawakami, and M Ohwada. Growth of native oxide on a silicon surface. Journal of Applied Physics, 68(3):1272-1281, 1990.

[235] Julien Proust, Frédéric Bedu, Bruno Gallas, Igor Ozerov, and Nicolas Bonod. All-dielectric colored metasurfaces with silicon mie resonators. ACS nano, 10(8):7761-7767, 2016.

[236] Shang Sun, Zhenxing Zhou, Chen Zhang, Yisheng Gao, Zonghui Duan, Shumin Xiao, and Qinghai Song. All-dielectric full-color printing with tio2 metasurfaces. ACS nano, 11(5):4445-4452, 2017. 
[237] Yusuke Nagasaki, Masafumi Suzuki, and Junichi Takahara. Alldielectric dual-color pixel with subwavelength resolution. Nano letters, 17(12):7500-7506, 2017.

[238] Thomas Wood, Meher Naffouti, Johann Berthelot, Thomas David, Jean-Benoît Claude, Léo Métayer, Anne Delobbe, Luc Favre, Antoine Ronda, Isabelle Berbezier, et al. All-dielectric color filters using sigebased mie resonator arrays. ACS photonics, 4(4):873-883, 2017.

[239] Vishal Vashistha, Gayatri Vaidya, Ravi S Hegde, Andriy E Serebryannikov, Nicolas Bonod, and Maciej Krawczyk. All-dielectric metasurfaces based on cross-shaped resonators for color pixels with extended gamut. ACS photonics, 4(5):1076-1082, 2017.

[240] Valentin Flauraud, Miguel Reyes, Ramon Paniagua-Dominguez, Arseniy I Kuznetsov, and Juergen Brugger. Silicon nanostructures for bright field full color prints. Acs Photonics, 4(8):1913-1919, 2017.

[241] Shang Sun, Wenhong Yang, Chen Zhang, Jixiang Jing, Yisheng Gao, Xiaoyi Yu, Qinghai Song, and Shumin Xiao. Real-time tunable colors from microfluidic reconfigurable all-dielectric metasurfaces. ACS nano, 12(3):2151-2159, 2018.

[242] Carl V Thompson. Solid-state dewetting of thin films. Annu. Rev. Mater. Sci., 42:399-434, 2012.

[243] Jongpil Ye and Carl V Thompson. Templated solid-state dewetting to controllably produce complex patterns. Adv. Mater., 23:1567-1571, 2011.

[244] Kahp Y Suh and Hong H Lee. Capillary force lithography: Large-area patterning, self-organization, and anisotropic dewetting. Adv. Funct. Mater., 12:405-413, 2002.

[245] Marco Abbarchi, Meher Naffouti, Benjamin Vial, Abdelmalek Benkouider, Laurent Lermusiaux, Luc Favre, Antoine Ronda, Sébastien Bidault, Isabelle Berbezier, and Nicolas Bonod. Wafer scale formation of monocrystalline silicon-based mie resonators via silicon-on-insulator dewetting. ACS nano, 8(11):11181-11190, 2014. 
[246] Meher Naffouti, Thomas David, Abdelmalek Benkouider, Luc Favre, Antoine Ronda, Isabelle Berbezier, Sebastien Bidault, Nicolas Bonod, and Marco Abbarchi. Fabrication of poly-crystalline si-based mie resonators via amorphous si on sio2 dewetting. Nanoscale, 8(5):2844-2849, 2016.

[247] Jongpil Ye, Dmitry Zuev, and Sergey Makarov. Dewetting mechanisms and their exploitation for the large-scale fabrication of advanced nanophotonic systems. International Materials Reviews, 64(8):439477, 2019.

[248] SK Sundaram and Eric Mazur. Inducing and probing non-thermal transitions in semiconductors using femtosecond laser pulses. Nature Mater., 1(4):217-224, 2002.

[249] Dieter W Bäuerle. Laser processing and chemistry. Springer Science \& Business Media, 2013.

[250] Jonas Berzinš, Simonas Indrišiūnas, Stefan Fasold, Michael Steinert, Olga Žukovskaja, Dana Cialla-May, Paulius Gečys, Stefan MB Bäumer, Thomas Pertsch, and Frank Setzpfandt. Laser-induced spatiallyselective tailoring of high-index dielectric metasurfaces. Optics Express, 28(2):1539-1553, 2020.

[251] Jonas Berzinš, Simonas Indrišiōnas, Koen van Erve, Arvind Nagarajan, Stefan Fasold, Michael Steinert, Giampiero Gerini, Paulius Gečys, Thomas Pertsch, Stefan MB Bäumer, et al. Direct and high-throughput fabrication of mie-resonant metasurfaces via single-pulse laser interference. ACS nano, 14(5):6138-6149, 2020.

[252] Georg Kucsko, Peter C Maurer, Norman Ying Yao, MICHAEL Kubo, Hyun Jong Noh, Po Kam Lo, Hongkun Park, and Mikhail D Lukin. Nanometre-scale thermometry in a living cell. Nature, 500(7460):54-58, 2013.

[253] Anastasia S Zalogina, RS Savelev, Elena V Ushakova, GP Zograf, FE Komissarenko, VA Milichko, SV Makarov, DA Zuev, and IV Shadrivov. Purcell effect in active diamond nanoantennas. Nanoscale, 10(18):8721-8727, 2018. 
[254] Nityanand Sharma, Jonathan Bar-David, Noa Mazurski, and Uriel Levy. Metasurfaces for enhancing light absorption in thermoelectric photodetectors. ACS Photonics, 2020.

[255] WA Challener, Chubing Peng, AV Itagi, D Karns, Wei Peng, Yingguo Peng, XiaoMin Yang, Xiaobin Zhu, NJ Gokemeijer, Y-T Hsia, et al. Heat-assisted magnetic recording by a near-field transducer with efficient optical energy transfer. Nature photonics, 3(4):220-224, 2009.

[256] Hamza K Khattak, Pablo Bianucci, and Aaron D Slepkov. Linking plasma formation in grapes to microwave resonances of aqueous dimers. Proceedings of the National Academy of Sciences, 116(10):4000-4005, 2019. 